Ann. Scient. Éc. Norm. Sup.,

$4^{\text {e }}$ série, t. 39, 2006, p. 197 à 243.

\title{
D-CHTOUCAS DE DRINFELD À MODIFICATIONS SYMÉTRIQUES ET IDENTITÉ DE CHANGEMENT DE BASE
}

\author{
PAR BAO CHÂU NGÔ
}

À Gérard Laumon pour son cinquantième anniversaire

RÉSUMÉ. - Nous proposons une nouvelle démonstration du lemme fondamental de changement de base pour $\mathrm{GL}_{n}$ pour certaines fonctions de Hecke en comparant la cohomologie de deux espaces de modules de $D$-chtoucas différents. Les démonstrations originelles, dues à Clozel et Labesse, utilisent la formule de traces et le cas d'unité dû à Kottwitz.

(c) 2006 Elsevier SAS

ABSTRACT. - We give a new proof of the base change fundamental lemma for $\mathrm{GL}_{n}$ for certain Hecke functions by comparing the cohomology of two different moduli spaces of $D$-shtukas. The original proofs, due to Clozel and Labesse, make use of the trace formula and of the unit case due to Kottwitz.

(c) 2006 Elsevier SAS

\section{Introduction}

Soit $F$ un corps local non-archimédien, $E$ une extension non-ramifiée de degré $r$ de $F$ et $\sigma$ un générateur de $\operatorname{Gal}(E / F)$. Soit $G=\mathrm{GL}_{d}$. Pour tous $\delta \in G(E)$, la classe de conjugaison de $\delta \sigma(\delta) \cdots \sigma^{r-1}(\delta)$ est $\sigma$-stable et définit donc une classe de conjugaison dans $G(F)$. Un élément $\gamma \in G(F)$ dans cette classe de conjugaison est appelé une norme de $\delta$. Supposons que $\gamma$ est semi-simple régulier.

Soit $\mathcal{H}_{F}$ l'algèbre des fonctions sphériques c'est-à-dire les fonctions complexes à support compacts sur $G(F)$ qui sont invariantes à gauche et à droite par $G\left(\mathcal{O}_{F}\right), \mathcal{O}_{F}$ étant l'anneau des entiers de $F$. D'après Satake, il existe un isomorphisme d'algèbres

$$
\mathcal{H}_{F} \stackrel{\sim}{\longrightarrow} \mathbb{C}\left[t_{1}^{ \pm 1}, \ldots, t_{d}^{ \pm 1}\right]^{\mathfrak{S}_{d}}
$$

où l'algèbre polynomiale $\mathbb{C}\left[t_{1}, \ldots, t_{d}\right]$ est l'algèbre des fonctions à support compact sur $T(F)$ qui sont invariantes par $T\left(\mathcal{O}_{F}\right), T$ étant le tore diagonal de $G$. De même, on a un isomorphisme

$$
\mathcal{H}_{E} \stackrel{\sim}{\longrightarrow} \mathbb{C}\left[u_{1}^{ \pm 1}, \ldots, u_{d}^{ \pm 1}\right]^{\mathfrak{S}_{d}} .
$$

L'homomorphisme de changement de base $\mathbf{b}: \mathcal{H}_{E} \rightarrow \mathcal{H}_{F}$ est alors défini par transport de ANNALES SCIENTIFIQUES DE L'ÉCOLE NORMALE SUPÉRIEURE 0012-9593/02/@ 2006 Elsevier SAS. Tous droits réservés. 
l'homomorphisme $u_{i} \mapsto t_{i}^{r}$ du côté des polynômes symétriques. Cet homomorphisme est compatible aux changements de base de la série principale non-ramifiée et est caractérisé par cette propriété.

Le lemme fondamental pour le changement de base affirme que l'intégrale d'une fonction sphérique $f$ sur une classe de conjugaison tordue $\delta$ est égale à l'intégrale de $\mathbf{b}(f)$ sur la norme $\gamma$ de $\delta$

$$
\mathbf{T} \mathbf{O}_{\delta}(f)=\mathbf{O}_{\gamma}(\mathbf{b}(f)) .
$$

Ce lemme a été établi pour la fonction unité $f=1_{G\left(\mathcal{O}_{E}\right)}$ par Kottwitz, puis pour toutes les fonctions sphériques par Arthur-Clozel dans le cas $\mathrm{GL}_{n}$. Pour un groupe réductif quelconque, le lemme fondamental pour le changement de base stable a été démontré par Clozel et Labesse du moins dans le cas $p$-adique. Leur méthode est basée sur l'usage de la formule des traces et le cas d'unité établi par Kottwitz.

Ce lemme fondamental pour le changement de base est un outil essentiel dans le comptage de points des variétés de Shimura ou des variétés modulaires de Drinfeld et la comparaison avec la formule des traces d'Arthur-Selberg. Le comptage naturel fait apparaître des intégrales orbitales tordues et le lemme est nécessaire pour les convertir en intégrales orbitales non tordues.

Dans ce travail, nous adoptons une stratégie inverse en partant du comptage des points dans les variétés modulaires de Drinfeld pour arriver au lemme fondamental. L'idée du départ est la suivante. Dans les variétés de Shimura avec mauvaise réduction, le comptage fait intervenir des fonctions de pondération provenant du modèle local des singularités qui est une sorte de sousvariétés de Schubert de variétés de drapeaux affines ou d'une grassmannienne affine [23]. On a par ailleurs une interprétation géométrique de l'homomorphisme de changement base $\mathbf{b}$ à l'aide des faisceaux pervers sur la grassmannienne affine [20]. Il s'agit donc de fabriquer un problème de module global tel que la construction [20] apparaisse comme son modèle local. On est conduit ainsi à étudier un espace de module des chtoucas à modifications multiples et l'action du groupe symétrique sur celles-ci. Une variante globale du lemme fondamental pour le changement de base prend la forme d'une comparaison du problème de module de chtoucas à modifications multiples et de la restriction à la Weil du problème de module des chtoucas à modifications simples. Nous ne faisons pas appel au cas d'unité établi par Kottwitz.

Signalons que E. Lau a indépendamment obtenu une démonstration de ce lemme fondamental, également à l'aide des chtoucas à modifications multiples mais par un argument différent.

Nous pensons que ce type de comparaison existe pour des chtoucas pour d'autres groupes réductifs, pourrait produire de nouvelles identités et établir certains cas de fonctorialité. Il faudra toutefois résoudre avant de nombreux problèmes notamment le comptage de $G$-chtoucas et la compactification des espaces de modules de $G$-chtoucas. Dans ce travail, on se restreint à des $\mathcal{D}$-chtoucas où $\mathcal{D}$ est une algèbre à division ayant beaucoup de places totalement ramifiées ce qui assure la compacité du problème de module. De plus, puisque le groupe $G=\mathcal{D}^{\times}$est une forme intérieure de $\mathrm{GL}_{n}$, le problème d'endoscopie disparaît ce qui simplifie considérablement le comptage des points.

Nous pensons aussi que ces constructions sont peut-être possibles même pour les variétés de Shimura car les fonctions de changement de bases sont aussi apparues sur les modèles locaux de Pappas-Rapoport, des variétés de Shimura pour des groupes unitaires en une place où le groupe unitaire est ramifié, voir [22].

Le résultat démontré dans cet article n'est pas nouveau, seule la démarche l'est. Vu son caractère un peu expérimental, nous avons voulu privilégier la clarté de l'exposé à la généralité

4e SÉRIE - TOME $39-2006-\mathrm{N}^{\circ} 2$ 
du résultat. On ne démontrera, dans ce texte, le lemme fondamental que pour les fonctions sphériques $f$ dont les transformés de Satake sont des fractions symétriques de degré $0^{1}$.

Passons en revue les différents chapitres de ce texte. On rappelle dans le chapitre 1 la définition des $\mathcal{D}$-chtoucas à modifications multiples, leur problème de module et son modèle local des singularités. La notion de chtoucas avec modifications multiples est due à Drinfeld et a été reprise récemment par Varshavsky. On donnera également un critère nécessaire pour que le morphisme caractéristique soit propre. Dans ce chapitre, on suit de près le livre de Lafforgue [14].

Sous l'hypothèse que le morphisme caractéristique est propre, les images directes du complexe d'intersection sont des systèmes locaux. On rappelle dans le chapitre 2 la définition de l'action des correspondances de Hecke sur ces systèmes locaux. On énonce dans 2.2 la description conjecturale de la somme alternée de ces systèmes locaux en suivant les conjectures de Langlands. Nous nous en servons comme un guide heuristique.

On énonce dans le paragraphe 3.3 le théorème principal de l'article, suivi d'un argument heuristique basé sur la description conjecturale de la somme alternée.

La démonstration de ce théorème est basée sur le comptage qui est le sujet du chapitre 4 . On y présente le comptage des chtoucas à modifications multiples, en suivant le comptage de Kottwitz des points des variétés de Shimura. Ce chapitre contient quelques innovations techniques et devrait se généraliser aux $G$-chtoucas pour un groupe réductif $G$ arbitraire.

On termine la démonstration du théorème 1 de 3.3 en 5.4 en comptant les points au-dessus des points cycliques et en évoquant le théorème de densité de Chebotarev. Le point un peu subtil de cette démonstration est la description de ces points cycliques en 5.2.

En spécialisant à la petite diagonale, on obtient une égalité où apparaît l'opérateur de changement de base $\mathbf{b}$. On déduit de cette égalité le lemme fondamental pour le changement de base pour les fonctions sphériques de degré 0 .

\section{Notation}

Soit $X$ une courbe projective lisse géométriquement connexe sur un corps fini $\mathbb{F}_{q}$. On note $F$ son corps des fonctions rationnelles. Pour tout schéma $S$, on note $|S|$ l'ensemble des points fermés de $S$. En particulier, pour $X$, l'ensemble $|X|$ est l'ensemble des valuations de $F$. Pour tout $x \in|X|$, on note $F_{x}$ le complété de $F, \mathcal{O}_{x}$ son anneau des entiers, $\kappa(x)$ le corps résiduel de $\mathcal{O}_{x}$. On note $\operatorname{deg}(x)$ le degré de l'extension $\left[\kappa(x): \mathbb{F}_{q}\right]$.

Soient $\mathbb{A}$ l'anneau des adèles de $F$ et $\mathcal{O}_{\mathbb{A}}$ son anneau des entiers. On a un homomorphisme degré $\mathbb{A}^{\times} \rightarrow \mathbb{Z}$ défini par

$$
\operatorname{deg}\left(a_{x}\right)_{x \in|X|}=\sum_{x} \operatorname{deg}(x) x\left(a_{x}\right) .
$$

On fixera une idèle $a \in \mathbb{A}^{\times}$de degré 1 .

On va fixer une clôture algébrique $k$ de $\mathbb{F}_{q}$. Si $S$ est un schéma sur $\mathbb{F}_{q}$, on va noter $\bar{S}=S \otimes_{\mathbb{F}_{q}} k$. En particulier, on a $\bar{X}=X \otimes_{\mathbb{F}_{q}} k$.

On fixera $D$ une algèbre à division centrale sur $F$, de dimension $d^{2}$. Soit $\mathcal{D}$ une $\mathcal{O}_{X}$-algèbre de fibre générique $D$ telle que $\mathcal{D}_{x}$ soit un ordre maximal de $D_{x}$ pour tout $x \in|X|$. Soit $X^{\prime}$ le plus grand ouvert de $X$ constitué des points $x \in|X|$ tels que $\mathcal{D}_{x}$ est isomorphe à $\mathfrak{g l}_{d}\left(\mathcal{O}_{x}\right)$.

\footnotetext{
${ }^{1}$ On référera à une version précédente de ce papier, disponible sur arXiv, où ce lemme est démontré pour toutes les fonctions de Hecke. E. Lau nous a informé d'une erreur dans cette ancienne version : le problème de module de $\mathcal{D}$-chtoucas n'est pas nécessairement compact si on ne suppose pas qu'il y a des places totalement ramifiées en grande quantité par rapport aux modifications. En augmentant le nombre de places totalement ramifiées, la généralisation à des fonctions sphériques de degré non nul reste valable. Cette généralisation est abandonnée ici à cause de sa longueur.
} 
Soit $G$ le schéma en groupes sur $X$ qui associe à tout schéma affine $\operatorname{Spec}(R)$ au-dessus de $X$ le groupe $\left(\mathcal{D} \otimes_{\mathcal{O}_{X}} R\right)^{\times}$. Puisque la fibre générique $D$ de $\mathcal{D}$ est une algèbre à division centrale sur $F$, le groupe $G_{F}$ est anisotropique c'est-à-dire qu'il ne contient aucun $F$-tore déployé non trivial. Au-dessus de $X^{\prime}$, le schéma en groupes $G$ est isomorphe à $X^{\prime} \times \mathrm{GL}_{d}$ localement pour la topologie de Zariski de $X^{\prime}$. Sur $X$ tout entier, il est isomorphe à $X \times \mathrm{GL}_{d}$ localement pour la topologie étale.

On utilisera souvent l'équivalence de Morita entre modules sur l'algèbre des matrices $M_{d}(K)$ et espace vectoriel sur un corps $K$. Ceci se fait comme d'habitude à l'aide d'un idempotent $e \in M_{d}$ qu'on va choisir une fois pour toutes. Ce choix est bénin dans la mesure où il n'intervient que dans les discussions sur les classes d'isomorphisme de modifications, qui finalement, ne dépendent pas de ce choix.

\section{D-chtoucas à modifications multiples}

\subsection{Modifications et grassmannienne affine}

Considérons le champ $\mathcal{D}$-Fib des $\mathcal{D}$-fibrés de rang 1 . Il associe à tout $\mathbb{F}_{q}$-schéma la catégorie en groupoïdes des $\mathcal{D} \otimes \mathcal{O}_{S}$-modules à droite, localement libre de rang 1. Ceux-ci sont en particulier des $\mathcal{O}_{X \times S}$-modules localement libres de rang $d^{2}$. Il est clair que $\mathcal{D}$-Fib est aussi le champ des $G$-torseurs localement triviaux pour la topologie étale de $X$, parce que $G$ est le schéma en groupes des automorphismes du $\mathcal{D}$-module trivial $\mathcal{D}$ et que tous les autres objets de la catégorie $\mathcal{D}$ - $\operatorname{Fib}(k)$ sont localement isomorphes à ce module trivial.

Soit $\bar{T}$ un sous-schéma fini de $\bar{X}^{\prime}$. Soient $\mathcal{V}$ et $\mathcal{V}^{\prime}$ deux $\mathcal{D}$-fibrés de rang 1 sur $\bar{X}$. Une $\bar{T}$-modification de $\mathcal{V}^{\prime}$ dans $\mathcal{V}$ est un isomorphisme entre les restrictions de $\mathcal{V}$ et de $\mathcal{V}^{\prime}$ à $\bar{X}-\bar{T}$

$$
t:\left.\left.\mathcal{V}^{\prime}\right|_{\bar{X}-\bar{T}} \stackrel{\sim}{\longrightarrow} \mathcal{V}\right|_{\bar{X}-\bar{T}} .
$$

Pour alléger les notations, notons $\mathcal{V}^{\bar{T}}$ et $\mathcal{V}^{\prime T}$ les restrictions de $\mathcal{V}$ et $\mathcal{V}^{\prime}$ à l'ouvert $\bar{X}-\bar{T}$. Une $\bar{T}$-modification sera alors un isomorphisme $t: \mathcal{V}^{\bar{T}} \stackrel{\sim}{\longrightarrow} \mathcal{V}^{\bar{T}}$.

Rappelons la définition de l'invariant d'une $\bar{T}$-modification. Soit $\bar{x} \in|\bar{T}|$ un point de $\bar{T}$. Notons $\mathcal{O}_{\bar{x}}$ le complété de $\mathcal{O}_{\bar{X}}$ en $\bar{x}$, et notons $F_{\bar{x}}$ son corps des fractions. Notons $V$ et $V^{\prime}$ les fibres génériques de $\mathcal{V}$ et $\mathcal{V}^{\prime}$ respectivement. La $\bar{T}$-modification $t$ permet d'identifier $V \stackrel{\sim}{\longrightarrow} V^{\prime}$ ainsi que leurs complétés en $\bar{x}$ qu'on note $V_{\bar{x}} \stackrel{\sim}{\longrightarrow} V_{\bar{x}}^{\prime}$. Le complété en $\bar{x}$ de $\mathcal{V}$ définit un $\mathcal{D}_{\bar{x}}$-réseau de $V_{\bar{x}}$, celui de $\mathcal{V}^{\prime}$ définit un $\mathcal{D}_{\bar{x}}$-réseau de $V_{\bar{x}}^{\prime}$. Puisque $\bar{T}$ est contenu dans $\bar{X}^{\prime}$ l'ouvert de non-ramification de $D$, il existe un isomorphisme $\mathcal{D}_{\bar{x}} \simeq M_{d}\left(\mathcal{O}_{\bar{x}}\right)$ qui induit un isomorphisme $D_{\bar{x}} \simeq M_{d}\left(F_{\bar{x}}\right)$. En utilisant l'idempotent standard $e \in M_{d}\left(\mathcal{O}_{\bar{x}}\right)$, on obtient un $F_{\bar{x}}$-espace vectoriel $e\left(V_{\bar{x}}\right)=e\left(V_{\bar{x}}^{\prime}\right)$ de dimension $d$ avec deux $\mathcal{O}_{\bar{x}}$-réseaux $e\left(\mathcal{V}_{\bar{x}}\right)$ et $e\left(\mathcal{V}_{\bar{x}}^{\prime}\right)$. La position relative de ces deux réseaux est donnée par une suite de $d$ entiers décroissants

$$
\lambda_{\bar{x}}=\left(\lambda^{1} \geqslant \lambda^{2} \geqslant \cdots \geqslant \lambda^{d}\right)
$$

d'après le théorème des diviseurs élémentaires. On pose $\operatorname{inv}_{\bar{x}}(t)=\lambda_{\bar{x}}$.

On peut donc associer à toute $\bar{T}$-modification $t: \mathcal{V}^{\bar{T}} \rightarrow \mathcal{V}^{\bar{T}^{\prime}}$ une fonction $\operatorname{inv}(t):|T| \rightarrow \mathbb{Z}_{+}^{d}$ à valeurs dans

$$
\mathbb{Z}_{+}^{d}=\left\{\lambda=\left(\lambda^{1}, \ldots, \lambda^{d}\right) \in \mathbb{Z}^{d} \mid \lambda^{1} \geqslant \cdots \geqslant \lambda^{d}\right\}
$$

$4^{\mathrm{e}}$ SÉRIE - TOME $39-2006-\mathrm{N}^{\circ} 2$ 
définie par $\bar{x} \mapsto \operatorname{inv}_{\bar{x}}(t)$. Il sera assez commode d'écrire formellement inv $(t)$ sous la forme

$$
\operatorname{inv}(t)=\sum_{\bar{x} \in \bar{T}} \operatorname{inv}_{\bar{x}}(t) \bar{x}
$$

En termes des $G$-torseurs, une $\bar{T}$-modification est un isomorphisme entre les restrictions de deux $G$-torseurs $\mathcal{V}$ et $\mathcal{V}^{\prime}$ à $\bar{X}-\bar{T}$. Dans un voisinage assez petit de $\bar{T}$ contenu dans $\bar{X}^{\prime}, G$ est isomorphe à $\mathrm{GL}_{d}$. Un tel isomorphisme étant choisi, on peut associer à une $\bar{T}$-modification de $G$-torseurs, une $\bar{T}$-modification de fibrés vectoriels de rang $d$. L'invariant défini ci-dessus coïncide avec l'invariant habituel associé à une $\bar{T}$-modification entre deux fibrés vectoriels de rang $d$ définis sur un voisinage arbitrairement petit de $\bar{T}$.

Nous allons noter $\mu$ et $\mu^{\vee} \in \mathbb{Z}_{+}^{d}$ les suites d'entiers

$$
\mu=(1,0, \ldots, 0) \quad \text { et } \quad \mu^{\vee}=(0, \ldots, 0,-1) .
$$

Elles sont parmi les éléments minimaux de $\mathbb{Z}_{+}^{d}$ par rapport à l'ordre partiel usuel, défini comme suit : pour tous $\lambda, \lambda^{\prime} \in \mathbb{Z}_{+}^{d}, \lambda \geqslant \lambda^{\prime}$ si et seulement si

$$
\begin{gathered}
\lambda^{1} \geqslant \lambda^{\prime 1}, \\
\vdots \\
\lambda^{1}+\cdots+\lambda^{d-1} \geqslant \lambda^{\prime 1}+\cdots+\lambda^{\prime d-1}, \\
\lambda^{1}+\cdots+\lambda^{d}=\lambda^{\prime 1}+\cdots+\lambda^{\prime d} .
\end{gathered}
$$

Nous aurons aussi besoin de la fonction croissante $\rho: \mathbb{Z}_{+}^{d} \rightarrow \mathbb{N}$ définie par

$$
\rho(\lambda)=\frac{1}{2}\left[(d-1) \lambda^{1}+(d-3) \lambda^{2}+\cdots+(1-d) \lambda^{d}\right]
$$

pour les calculs de dimension. On a, en particulier, $2 \rho(\mu)=2 \rho\left(\mu^{\vee}\right)=d-1$.

Les modifications de position $\mu$ et $\mu^{\vee}$ correspondent bien aux notions usuelles de modifications supérieures et inférieures de fibrés vectoriels de rang $d$. Soit $\bar{u} \in X^{\prime}(k)$; prenons $\bar{T}=\{\bar{u}\}$. Une $\bar{T}$-modification de $\mathcal{D}$-fibrés $t: \mathcal{V}^{\bar{T}} \rightarrow \mathcal{V}^{\prime \bar{T}}$ d'invariant $\mu$ se prolonge en une injection $\mathcal{D} \otimes_{\mathbb{F}_{q}} k$-linéaire $\mathcal{V} \rightarrow \mathcal{V}^{\prime}$ dont le conoyau est un $k$-espace vectoriel de rang $d$, supporté par $\bar{u}$. Si de plus, on a choisi un voisinage assez petit de $\bar{u}$ sur lequel il existe un isomorphisme entre $G$ et $\mathrm{GL}_{d}$, une $\bar{T}$-modification de $\mathcal{D}$-fibrés $t: \mathcal{V}^{\bar{T}} \rightarrow \mathcal{V}^{\prime \bar{T}}$ de position $\mu$ induit, sur ce voisinage, une injection de fibrés vectoriels de rang $d$ dont le conoyau est un $k$-espace vectoriel de dimension 1 , supporté par $\bar{u}$. Idem, les $\bar{T}$-modifications d'invariant $\mu^{\vee}$ correspondent aux modifications inférieures de fibrés vectoriels de rang $d$.

DÉFInition 1. - Soit $\lambda \in \mathbb{Z}_{+}^{d}$. Le champ $\mathcal{D}$ - Hck $_{\lambda}$ associe à tout $\mathbb{F}_{q}$-schéma $S$ la catégorie en groupoïdes des données $\left(u, \mathcal{V}, \mathcal{V}^{\prime}\right)$ comprenant :

- un point $u$ de $X^{\prime}$ à valeurs dans $S$,

- deux points $\mathcal{V}, \mathcal{V}^{\prime}$ de $\mathcal{D}$-Fib à valeurs dans $S$,

- un isomorphisme

$$
t:\left.\left.\mathcal{V}^{\prime}\right|_{X \times S-\Gamma(u)} \stackrel{\sim}{\longrightarrow} \mathcal{V}\right|_{X \times S-\Gamma(u)}
$$

entre les restrictions de $\mathcal{V}$ et $\mathcal{V}^{\prime}$ au complémentaire dans $X \times S$ du graphe $\Gamma(u)$ de $u: S \rightarrow X$, 
telles que pour tout $\bar{s} \in S(k)$, pour $\bar{u}=u(\bar{s})$, au-dessus de $\bar{s}$, la modification $t_{\bar{s}}: \mathcal{V}^{\prime} \frac{\{\bar{u}\}}{\longrightarrow} \underset{\mathcal{S}}{\{} \mathcal{V}_{\bar{s}}^{\{\bar{u}\}}$ a un invariant $\operatorname{inv}_{\bar{u}}\left(t_{\bar{s}}\right) \leqslant \lambda$.

On va aussi considérer le sous-champ $\mathcal{D}-\mathrm{Hck}_{\lambda}^{\prime}$ de $\mathcal{D}-\mathrm{Hck}_{\lambda}$ classifiant les mêmes données, mais en imposant une condition plus forte sur la modification $t$ : on demande qu'en tout point géométrique $\bar{s} \in S(k)$, la $\{u(\bar{s})\}$-modification $t_{\bar{s}}$ a l'invariant $\operatorname{inv}_{u(\bar{s})}(t)=\lambda$.

Si $\lambda$ est un élément minimal de $\mathbb{Z}_{+}^{d}$, comme $\mu$ ou $\mu^{\vee}$, il est clair que $\mathcal{D}$ - $\operatorname{Hck}_{\lambda}^{\prime}=\mathcal{D}$ - Hck $_{\lambda}$.

PROPOSITION 2. - Le morphisme

$$
\mathcal{D}-\mathrm{Hck}_{\lambda} \rightarrow X^{\prime} \times \mathcal{D} \text { - Fib }
$$

qui associe $\left(u ; \mathcal{V}, \mathcal{V}^{\prime} ; t\right) \mapsto(u, \mathcal{V})$ est un morphisme représentable et projectif. De plus, $\mathcal{D}$ - Hck ${ }_{\lambda}^{\prime}$ est un ouvert dense de $\mathcal{D}$ - $\mathrm{Hck}_{\lambda}$, lisse et purement de dimension relative $2 \rho(\lambda)$ sur $X^{\prime} \times \mathcal{D}$-Fib.

En particulier, si $\lambda$ est égal à $\mu$ où $\mu^{\vee}$, le morphisme $\mathcal{D}$ - $\mathrm{Hck}_{\lambda} \rightarrow X^{\prime} \times \mathcal{D}$-Fib est projectif, lisse et purement de dimension relative $d-1$.

Pour un $\lambda$ non minuscule, $\mathcal{D}$ - $\mathrm{Hck}_{\lambda}$ n'est pas nécessairement lisse au-dessus de $X^{\prime} \times \mathcal{D}$-Fib. Toutefois, il est lisse au-dessus de $\mathcal{Q}_{\lambda} \times \mathcal{D}$-Fib où $\mathcal{Q}_{\lambda}$ est une variante globale de la grassmannienne affine, introduite par Beilinson et Drinfeld [3]. Rappelons la définition de $\mathcal{Q}_{\lambda}$.

Soit $Q$ un $\mathcal{O}_{\bar{X}}$-module supporté par un point $u \in \bar{X}$. Comme $\mathcal{O}_{u}$-module, il est isomorphe à un module de la forme

$$
Q \simeq \mathcal{O}_{u} / \varpi_{u}^{\lambda^{1}} \oplus \cdots \oplus \mathcal{O}_{u} / \varpi_{u}^{\lambda^{d^{\prime}}}
$$

où la suite décroissante d'entiers naturels $\lambda^{1} \geqslant \cdots \geqslant \lambda^{d^{\prime}}>0$ est bien définie. Ici, $\varpi_{u}$ est un uniformisant de $\bar{X}$ en $u$. On ne va considérer que les suites de longueur $d^{\prime} \leqslant d$ lesquelles seront complétées par un nombre nécessaire de 0 pour devenir un élément $\lambda \in \mathbb{N}_{+}^{d}$ c'est-à-dire

$$
\lambda=\left(\lambda^{1} \geqslant \lambda^{2} \geqslant \cdots \geqslant \lambda^{d} \geqslant 0\right) .
$$

On notera alors $\operatorname{inv}(Q)=\lambda$.

DÉfInITION 3. - Pour tout $\lambda \in \mathbb{N}_{+}^{d}$, le champ $\mathcal{Q}_{\lambda}$ qui associe à tout $\mathbb{F}_{q}$-schéma $S$ la catégorie en groupoïdes des données suivantes :

- un morphisme $u: S \rightarrow X^{\prime}$,

- un $\mathcal{D}_{X} \otimes \mathcal{O}_{S}$-module $Q, \mathcal{O}_{S}$-plat, supporté par le graphe de $u$ tel que pour tout point géométrique $s \in S(k)$, pour $u=u(s) \in \bar{X}^{\prime}$, pour tout isomorphisme $\mathcal{D}_{u} \stackrel{\sim}{\longrightarrow} M_{d}\left(\mathcal{O}_{u}\right)$, audessus de $s, e(Q)$ est un module de torsion supporté par $u$, d'invariant $\operatorname{inv}(Q) \leqslant \lambda$. Ici, $e$ est l'idempotent standard de $M_{d}\left(\mathcal{O}_{u}\right)$ qu'on a transféré à $\mathcal{D}_{u}$.

Proposition 4. - Soit $\lambda \in \mathbb{N}_{+}^{d}$. Soit

$$
\left(u ; \mathcal{V}, \mathcal{V}^{\prime} ; t: \mathcal{V}^{\{u\}} \stackrel{\sim}{\longrightarrow} \mathcal{V}^{\prime\{u\}}\right)
$$

un objet de $\mathcal{D}$ - $\operatorname{Hck}_{\lambda}(S)$. Alors t se prolonge de façon unique en une injection $\mathcal{D}_{X} \otimes \mathcal{O}_{S}$-linéaire $t: \mathcal{V} \hookrightarrow \mathcal{V}^{\prime}$. De plus, si $Q$ désigne le conoyau de cette injection, la paire $(u, Q)$ est un objet de $\mathcal{Q}_{\lambda}(S)$.

Le morphisme

$$
\mathcal{D}-\mathrm{Hck}_{\lambda} \rightarrow \mathcal{Q}_{\lambda} \times \mathcal{D} \text { - Fib }
$$

qui associe à $\left(u ; \mathcal{V}, \mathcal{V}^{\prime} ; t\right)$ la paire $(u, Q) \in \mathcal{Q}_{\lambda}$ et le $\mathcal{D}$-fibré $\mathcal{V} \in \mathcal{D}$-Fib, est un morphisme lisse. Il en est de même du morphisme $\left(u ; \mathcal{V}, \mathcal{V}^{\prime} ; t\right) \mapsto\left(u ; Q, \mathcal{V}^{\prime}\right)$.

$4^{\mathrm{e}}$ SÉRIE - TOME $39-2006-\mathrm{N}^{\circ} 2$ 
Démonstration. - Pour démontrer que le morphisme

$$
f:\left(u ; \mathcal{V}, \mathcal{V}^{\prime} ; t\right) \mapsto\left(u ; Q, \mathcal{V}^{\prime}\right)
$$

est lisse, il suffit de démontrer qu'il est le composé d'une immersion ouverte suivie de la projection d'un fibré vectoriel sur sa base. La modification étant locale dans un voisinage de $u$, on peut remplacer un $\mathcal{D}$-module par un fibré vectoriel de rang $n$. Étant donné un fibré vectoriel de rang $n$ et un $\mathcal{O}$-module de torsion $Q$ de type $\lambda$, la fibre du morphisme $f$ consiste en des applications $\mathcal{O}$-linéaires surjectives $\mathcal{V}^{\prime} \rightarrow Q$. En omettant la surjectivité, la fibre est clairement un espace vectoriel de dimension fixe. Quant à la condition de surjectivité, c'est une condition ouverte.

Il n'est pas difficile d'étendre la définition de $\mathcal{Q}_{\lambda}$ et la proposition ci-dessus à un élément $\lambda \in \mathbb{Z}_{+}^{d}$ arbitraire. Soit $\lambda \in \mathbb{Z}_{+}^{d}$ et soit $N \in \mathbb{N}$ un entier assez grand tel que $\lambda+N=\left(\lambda^{1}+N \geqslant\right.$ $\cdots \geqslant \lambda^{d}+N \geqslant 0$ ). On va poser formellement $\mathcal{Q}_{\lambda}=\mathcal{Q}_{\lambda+N}$. Ceci ne dépend pas de l'entier $N$ pourvu que $\lambda_{d}+N \geqslant 0$. Si $t$ est une modification en $u$ de $\mathcal{V}^{\prime}$ dans $\mathcal{V}$ de position $\lambda, t$ induit une modification en $u$ de $\mathcal{V}$ dans $\mathcal{V}^{\prime}[N]$ de type $\lambda+N$. La proposition s'étend donc sans difficultés au cas $\lambda \in \mathbb{Z}_{+}^{d}$.

L'énoncé suivant utilise le lien entre $\mathcal{Q}_{\lambda}$ et les cellules de la grassmannienne affine introduite par Lusztig [18], voir [7,19,21].

PROPOSITION 5. - Il existe une variété projective $\widetilde{\mathcal{Q}}_{\lambda}$ telle que localement pour la topologie étale de $X^{\prime}$, il existe des $X^{\prime}$-morphismes lisses surjectifs de $X^{\prime} \times \widetilde{\mathcal{Q}}_{\lambda}$ sur $\mathcal{Q}_{\lambda}$.

Démonstration. - Suivant Mirkovic et Vilonen [19], on considère le foncteur $\widetilde{\mathcal{Q}}_{\lambda, X^{\prime}}$ qui associe à tout $\mathbb{F}_{q}$-schéma $S$ l'ensemble des données $(u, \mathcal{V}, t)$

- $u$ est un point de $X^{\prime}$ à valeur dans $S$,

- $\mathcal{V}$ est un point de $\mathcal{D}$ - Fib à valeur dans $S$,

- $t: \mathcal{D}^{\{u\}} \rightarrow \mathcal{V}^{\{u\}}$ est une modification en $u$ d'invariant $\operatorname{inv}_{u}(t) \leqslant \lambda$, du $\mathcal{D}$-fibré trivial $\mathcal{D}$ dans $\mathcal{V}$.

Ce foncteur est représentable par un schéma projectif au-dessus de $X^{\prime}$. D'après la proposition précédente, on sait que le morphisme $\widetilde{\mathcal{Q}}_{\lambda, X^{\prime}} \rightarrow \mathcal{Q}_{\lambda}$ est un morphisme lisse. Il n'est pas difficile de vérifier qu'il est surjectif.

Si $Y^{\prime} \rightarrow X^{\prime}$ est un revêtement étale, il est clair que

$$
\widetilde{\mathcal{Q}}_{\lambda, Y^{\prime}}=\widetilde{\mathcal{Q}}_{\lambda, X^{\prime}} \times_{X^{\prime}} Y^{\prime} .
$$

Localement pour la topologie étale, on peut supposer que $Y^{\prime}$ est isomorphe à la droite affine. Puisque $Y^{\prime} \simeq \mathbb{A}^{1}$, on a une action par translation de $\mathbb{A}^{1}$ sur $\widetilde{\mathcal{Q}}_{\lambda, X}$ qui permet d'obtenir un isomorphisme $\widetilde{\mathcal{Q}}_{\lambda, Y^{\prime}} \simeq Y^{\prime} \times \widetilde{\mathcal{Q}}_{\lambda}$ où $\widetilde{\mathcal{Q}}_{\lambda}$ est une fibre quelconque de $\widetilde{\mathcal{Q}}_{\lambda, Y^{\prime}}$ au-dessus de $Y^{\prime}$.

COROLlaire 6. - Soit $\mathcal{A}_{\lambda}$ le complexe d'intersection de $\mathcal{Q}_{\lambda}$. Il satisfait deux propriétés :

(1) Il est localement acyclique par rapport au morphisme $\mathcal{Q}_{\lambda} \rightarrow X^{\prime}$.

(2) Ses restrictions aux fibres de $\mathcal{Q}_{\lambda}$ sur $X^{\prime}$ sont, à décalage près, des faisceaux pervers irréductibles.

Démonstration. - Localement pour la topologie étale de $X^{\prime}$, il existe un $X^{\prime}$-morphisme lisse surjectif $X^{\prime} \times \widetilde{\mathcal{Q}}_{\lambda} \rightarrow \mathcal{Q}_{\lambda}$. On se ramène donc à démontrer l'énoncé pour $X^{\prime} \times \widetilde{\mathcal{Q}}_{\lambda}$ où il se déduit de la formule de Künneth.

D'après un théorème de Lusztig, les faisceaux pervers $\mathcal{A}_{\lambda}$ sur $\mathcal{Q}_{\lambda}$ réalisent via le dictionnaire faisceaux-fonctions une base de l'algèbre de Hecke ayant des propriétés remarquables. Pour préciser cet énoncé dont on va se servir, rappelons la définition des algèbres de Hecke. 
Soit $x$ un point fermé de $X^{\prime}$. On définit $\mathcal{H}_{x}$ comme l'algèbre des fonctions à valeurs dans $\mathbb{Q}_{\ell}$, à support compact dans $G\left(F_{x}\right)$ qui sont invariantes à gauche et à droite par $G\left(\mathcal{O}_{x}\right)$. La structure d'algèbre de $\mathcal{H}_{x}$ est définie par le produit de convolution usuelle

$$
\left(f * f^{\prime}\right)(g)=\int_{G\left(F_{x}\right)} f(h) f^{\prime}\left(h^{-1} g\right) d g
$$

en intégrant par rapport à la mesure de Haar $d g \operatorname{sur} G\left(F_{x}\right)$, normalisée par $\operatorname{vol}\left(G\left(\mathcal{O}_{x}\right)\right)=1$.

Pour tout $x \in\left|X^{\prime}\right|, G$ est isomorphe à $\mathrm{GL}_{d}$ dans un voisinage de Zariski de $x$ de sorte que $\mathcal{H}_{x}$ est isomorphe à l'algèbre de Hecke de $\mathrm{GL}_{d}$. L'algèbre de Hecke de $\mathrm{GL}_{d}$ admet donc une base évidente $\left(\phi_{\lambda}\right)$, paramétrée par les $\lambda \in \mathbb{Z}_{+}^{d}$; la fonction $\phi_{\lambda}$ étant la fonction caractéristique de la double classe de $\lambda(x)$

$$
\operatorname{GL}_{d}\left(\mathcal{O}_{x}\right) \operatorname{diag}\left(\varpi_{x}^{\lambda_{1}}, \ldots, \varpi_{x}^{\lambda_{n}}\right) \mathrm{GL}_{d}\left(\mathcal{O}_{x}\right)
$$

où $\varpi_{x}$ est une uniformisante de $F_{x}$. Transférée à $G$, on obtient une base de $\mathcal{H}_{x}$. Il n'est pas difficile de montrer que la base ainsi obtenue ne dépend ni du choix de l'isomorphisme entre $G_{x}$ et $\mathrm{GL}_{d}$, ni du choix de l'uniformisante $\varpi_{x}$.

L'isomorphisme de Satake permet d'identifier canoniquement l'algèbre $\mathcal{H}_{x}$ avec l'algèbre $\mathbb{Q}_{\ell}[\widehat{G}]^{\operatorname{Ad}(\widehat{G})}$ des fonctions polynomiales

$$
\hat{f}: \widehat{G}\left(\mathbb{Q}_{\ell}\right) \rightarrow \mathbb{Q}_{\ell}
$$

qui sont invariantes par l'action adjointe de $\widehat{G}\left(\mathbb{Q}_{\ell}\right)$. Dans la situation présente, le groupe dual de Langlands $\widehat{G}$ est aussi égal à $\mathrm{GL}_{d}$. Pour tout $\lambda \in \mathbb{Z}_{+}^{d}$, notons $\hat{\psi}_{\lambda}$ la fonction définie par la trace de tout élément $\hat{g} \in \widehat{G}\left(\mathbb{Q}_{\ell}\right)$ sur la représentation irréductible de $\widehat{G}$ de plus haut poids $\lambda$. Ces fonctions forment une base de $\mathbb{Q}_{\ell}[\widehat{G}]^{\operatorname{Ad}(\widehat{G})}$ lorsque $\lambda$ parcourt $\mathbb{Z}_{+}^{d}$. Le transformé de Satake $\hat{\phi}_{\lambda}$ de la fonction double classe $\phi_{\lambda}$ diffère en général de $\hat{\psi}_{\lambda}$. Le faisceau pervers $\mathcal{A}_{\lambda}$ tient compte précisément de cette différence.

Supposons $\operatorname{deg}(x)=s$. Les $k$-points du champ $\mathcal{Q}_{\lambda}$ au-dessus de $x$, en nombre fini, tous définis sur $\mathbb{F}_{q^{s}}$, correspondent bijectivement aux éléments $\alpha \in \mathbb{Z}_{+}^{d}$ tels que $\alpha \leqslant \lambda$. On les note $\alpha(x) \in \mathcal{Q}_{\lambda}(x)$. Soit $P_{\lambda, \alpha}\left(q^{s}\right)$ la trace de Frob $_{q^{s}}$ sur la fibre de $\mathcal{A}_{\lambda}$ au-dessus de $\alpha(x)$.

THÉORÈME 7 (Lusztig-Kato). - La fonction

$$
\psi_{\lambda}=\sum_{\alpha \leqslant \lambda} P_{\lambda, \alpha}\left(q^{s}\right) \phi_{\alpha},
$$

dans $\mathcal{H}_{x}$, a pour transformé de Satake la fonction $\hat{\psi}_{\lambda}$ définie par la trace sur la représentation irréductible de plus haut poids $\lambda$ de $\widehat{G}\left(\mathbb{Q}_{\ell}\right)$.

On renvoie à [18] pour la démonstration de ce théorème et à [7,19] pour son interprétation géométrique.

\subsection{Modifications itérées et propriété de factorisation}

On aura aussi besoin de considérer des suites de modifications avec les invariants bornés. Soient $n$ un entier naturel et $\underline{\lambda}=\left(\lambda_{1}, \ldots, \lambda_{n}\right)$ un $n$-uple d'éléments de $\mathbb{Z}_{+}^{d}$.

DÉFINITION 1. - Le champ $\mathcal{D}$ - Hck $\mathrm{H}_{\underline{\lambda}}$ associe à tout $\mathbb{F}_{q}$-schéma $S$ la catégorie en groupoïdes des données $\left(\left(u_{j}\right)_{j=1}^{n},\left(\mathcal{V}_{j}\right)_{j=0}^{n},\left(t_{j}\right)_{j=1}^{n}\right)$ comprenant

$4^{\text {e }}$ SÉRIE - TOME $39-2006-\mathrm{N}^{\circ} 2$ 
- $n$ points $u_{1}, \ldots, u_{n}$ de $X^{\prime}$ à valeurs dans $S$,

- $n+1$ points $\mathcal{V}_{0}, \ldots, \mathcal{V}_{n}$ de $\mathcal{D}$-Fib à valeurs dans $S$,

- pour tous $j=1, \ldots, n$, un isomorphisme

$$
t_{j}:\left.\left.\mathcal{V}_{j}\right|_{X \times S-\Gamma\left(u_{j}\right)} \stackrel{\sim}{\longrightarrow} \mathcal{V}_{j-1}\right|_{X \times S-\Gamma\left(u_{j}\right)}
$$

entre les restrictions de $\mathcal{V}_{j-1}$ et $\mathcal{V}_{j}$ au complémentaire dans $X \times S$ du graphe $\Gamma\left(u_{i}\right)$ de $u_{j}: S \rightarrow X$ telles que pour tout $\bar{s} \in S(k)$, pour $\bar{u}_{j}=u_{j}(\bar{s}) \in \bar{X}^{\prime}$, au-dessus de $\bar{s}$, la modification $t_{j}: \mathcal{V}_{j}^{\left\{\bar{u}_{j}\right\}} \stackrel{\sim}{\longrightarrow} \mathcal{V}_{j-1}^{\left\{\bar{u}_{j}\right\}}$ a l'invariant $\operatorname{inv}_{\bar{u}_{j}}\left(t_{j}\right) \leqslant \lambda_{j}$.

Il est clair que $\mathcal{D}$ - Cht $_{\underline{\lambda}}$ peut être construit au moyen de produit fibré successif. Pour tout $j=1, \ldots, n$, si on note $p_{j}$ et $p_{j}^{\prime}$ les deux projections de $\mathcal{D}$ - $\mathrm{Cht}_{\lambda_{j}}$ sur $\mathcal{D}$-Fib qui envoie $\left(u, \mathcal{V}, \mathcal{V}^{\prime}, t\right)$ sur $\mathcal{V}$ et $\mathcal{V}^{\prime}$ respectivement, alors on a

$$
\mathcal{D}-\mathrm{Cht}_{\underline{\underline{\lambda}}}=\mathcal{D}-\mathrm{Cht}_{\lambda_{1}} \times_{p_{1}^{\prime}, \mathcal{D}-\mathrm{Fib}, p_{2}} \cdots \times_{p_{n-1}^{\prime}, \mathcal{D}-\mathrm{Fib}, p_{n}} \mathcal{D}-\mathrm{Cht}_{\lambda_{n}} \text {. }
$$

On va aussi considérer le sous-champ $\mathcal{D}$ - $\mathrm{Hck}_{\lambda}^{\prime}$ de $\mathcal{D}$ - $\mathrm{Hck}_{\lambda}$ classifiant les mêmes données mais en imposant une condition plus forte sur les modifications $t_{i}$ en demandant que $\operatorname{inv}_{\bar{u}_{j}}\left(t_{j}\right)=\lambda_{j}$.

Proposition 2. - Soit $\underline{\lambda}=\left(\lambda_{1}, \ldots, \lambda_{n}\right)$ une suite de $n$ éléments de $\mathbb{Z}_{+}^{d}$. Le morphisme

$$
\mathcal{D}-\mathrm{Hck}_{\underline{\lambda}} \rightarrow X^{\prime n} \times \mathcal{D} \text { - Fib }
$$

qui associe $\left(\left(u_{j}\right)_{j=1}^{n},\left(\mathcal{V}_{j}\right)_{j=0}^{n},\left(t_{j}\right)_{j=1}^{n}\right) \mapsto\left(\left(u_{j}\right), \mathcal{V}_{0}\right)$ est un morphisme représentable et projectif. De plus, $\mathcal{D}$ - $\mathrm{Hck}_{\lambda}^{\prime}$ est un ouvert dense de $\mathcal{D}$ - $\mathrm{Hck}_{\underline{\lambda}}$, lisse et purement de dimension relative $\sum_{j=1}^{n} 2 \rho\left(\lambda_{j}\right)$ sur $X^{i^{n}} \times \mathcal{D}$ - Fib.

Démonstration. - On se ramène au cas d'une seule modification au moyen du produit fibré successif, mentionné ci-dessus.

Sur l'ouvert complémentaire de la réunion des diagonales de $X^{\prime n}$, on aura un vrai produit fibré.

Proposition 3. - Au-dessus de l'ouvert U complémentaire de la réunion des diagonales dans $X^{\prime n}, \mathcal{D}$-Hck $\mathrm{H}_{\underline{\lambda}}$ est canoniquement isomorphe au produit fibré des morphismes $p_{j}: \mathcal{D}$ - $\operatorname{Hck}_{\lambda_{j}} \rightarrow \mathcal{D}$-Fib pour $j=1, \ldots, n$.

Démonstration. - Il s'agit d'une propriété bien connue des modification de fibrés vectoriels. Soit $x_{1}, x_{2}$ deux points distincts de $\bar{X}$. Fixer un fibré vectoriel $\mathcal{V}$ sur $\bar{X}$. Alors donner une «grande » modification

$$
t: \mathcal{V}^{\prime\left\{x_{1}, x_{2}\right\}} \stackrel{\sim}{\longrightarrow} \mathcal{V}^{\left\{x_{1}, x_{2}\right\}}
$$

telle que $\operatorname{inv}_{x_{j}}(t)=\lambda_{j}$ est équivalente à donner deux modifications indépendantes

$$
t_{1}^{\prime}: \mathcal{V}_{1}^{x_{1}} \stackrel{\sim}{\longrightarrow} \mathcal{V}^{x_{1}} \quad \text { et } \quad t_{2}^{\prime}: \mathcal{V}_{2}^{\prime x_{2}} \stackrel{\sim}{\longrightarrow} \mathcal{V}^{x_{2}}
$$

telles que $\operatorname{inv}_{x_{j}}\left(t_{j}^{\prime}\right)=\lambda_{j}$, ou encore équivalente à deux modifications «en itération »

$$
t_{2}: \mathcal{V}_{2}^{x_{2}} \rightarrow \mathcal{V}_{1}^{x_{2}} \quad \text { et } \quad t_{1}: \mathcal{V}_{1}^{x_{1}} \rightarrow \mathcal{V}^{x_{1}}
$$

avec les mêmes invariants. L'adaptation est évidente au cas de $\mathcal{D}$-fibrés si on suppose en plus que $x_{1}, x_{2}$ sont dans $X^{\prime}$. La proposition s'en déduit. 
Tout comme dans le cas d'une seule modification, on veut construire un objet «minimal» qui capture toutes les singularités du morphisme $\mathcal{D}$ - $\mathrm{Hck}_{\underline{\lambda}} \rightarrow X^{\prime n} \times \mathcal{D}$-Fib.

DÉfinition 4. - Supposons que $\lambda_{1}, \ldots, \lambda_{n} \in \mathbb{N}_{+}^{d}$. Le champ $\mathcal{Q}_{\underline{\lambda}}$ qui associe à tout $\mathbb{F}_{q^{-}}$ schéma la catégorie en groupoïdes des données suivantes :

- $n$ points $u_{1}, \ldots, u_{n}$ de $X^{\prime}$ à valeurs dans $S$,

- un drapeau de $\mathcal{D}_{X} \otimes \mathcal{O}_{S}$-modules de torsion, $\mathcal{O}_{S}$-plats

$$
0=Q_{0} \subset Q_{1} \subset Q_{2} \subset \cdots \subset Q_{n}
$$

tel que $\left(u_{j}, Q_{i} / Q_{j-1}\right)$ est un point de $\mathcal{Q}_{\lambda_{j}}$ à valeur dans $S$.

Proposition 5. - Soit $\lambda \in \mathbb{N}_{+}^{d}$. Soit

$$
\left(\left(u_{j}\right)_{j=1}^{n} ;\left(\mathcal{V}_{j}\right)_{j=0}^{n}, \mathcal{V}_{j}^{\left\{u_{j}\right\}} \stackrel{t_{j}}{\longrightarrow} \mathcal{V}_{j-1}^{\left\{u_{j}\right\}}\right)
$$

un objet de $\mathcal{D}-\operatorname{Hck}_{\underline{\underline{\lambda}}}(S)$. Alors $t_{j}$ se prolonge de façon unique en une injection $\mathcal{D}_{X} \otimes \mathcal{O}_{S}$-linéaire $t_{j}: \mathcal{V}_{j} \hookrightarrow \mathcal{V}_{j-1}$. De plus, si $Q_{j}$ désigne le conoyau de

$$
t_{j} \circ \cdots \circ t_{1}: \mathcal{V}_{j} \rightarrow \mathcal{V}_{0}
$$

alors $\left(\left(u_{j}\right)_{j=1}^{n}, Q_{1} \subset \cdots \subset Q_{n}\right)$ est un objet de $\mathcal{Q}_{\underline{\lambda}}(S)$.

Le morphisme

$$
\mathcal{D}-\mathrm{Hck}_{\underline{\lambda}} \rightarrow \mathcal{Q}_{\underline{\lambda}} \times \mathcal{D} \text { - Fib }
$$

qui fait associer à $\left(\left(u_{j}\right) ;\left(\mathcal{V}_{j}\right),\left(t_{j}\right)\right)$ la paire $\left(\left(u_{j}\right),\left(Q_{j}\right)\right) \in \mathcal{Q}_{\underline{\lambda}}$ comme ci-dessus, et le $\mathcal{D}$-fibré $\mathcal{V}_{0} \in \mathcal{D}$-Fib, est un morphisme lisse.

Démonstration. - On se ramène par récurence au cas $n=1$, donc à la proposition 2 de 1.1, en utilisant la description $\mathcal{D}-\mathrm{Hck}_{\underline{\lambda}}$ comme un «produit fibré successif », qui se trouve après la définition 1.

La définition 4 et la proposition 5 se généralisent sans difficulté à tous $\lambda_{1}, \ldots, \lambda_{n} \in \mathbb{Z}_{+}^{d}$.

Proposition 6. - Le complexe d'intersection $\mathcal{A}_{\underline{\lambda}}$ de $\mathcal{Q}_{\underline{\lambda}}$ a les propriétés suivantes.

(1) Il est localement acyclique par rapport au morphisme $\mathcal{Q}_{\lambda} \rightarrow X^{n}$.

(2) Ses restrictions aux fibres de ce morphisme sont des faisceaux pervers irréductibles.

Démonstration. - Essentiellement la même démonstration que 1.1.6 marche.

On a de plus une propriété de factorisation.

Proposition 7. - Au-dessus de l'ouvert $U$ complémentaire de la réunion des diagonales dans $X^{\prime n}, \mathcal{Q}_{\underline{\lambda}}$ est isomorphe à $\mathcal{Q}_{\lambda_{1}} \times \cdots \times \mathcal{Q}_{\lambda_{n}}$. Via cet isomorphisme, $\mathcal{A}_{\underline{\lambda}}$ s'identifie à $\mathcal{A}_{\lambda_{1}} \otimes \cdots \otimes \overline{\mathcal{A}}_{\lambda_{n}}$.

Démonstration. - Il suffit de combiner la propriété de factorisation avec la formule de Künneth.

\section{3. $\mathcal{D}$-chtoucas à modifications multiples}

La notion de chtoucas à modifications multiples est due à Drinfeld [5] et est récemment reprise dans un article de Varshavsky [26].

$4^{e}$ SÉRIE - TOME $39-2006-\mathrm{N}^{\circ} 2$ 
Soit $\underline{\lambda}=\left(\lambda_{1}, \ldots, \lambda_{n}\right)$ une suite de $n$ éléments de $\mathbb{Z}_{+}^{d}$. On définit le champ $\mathcal{D}$ - Cht $t_{\underline{\lambda}}$ en formant le produit cartésien

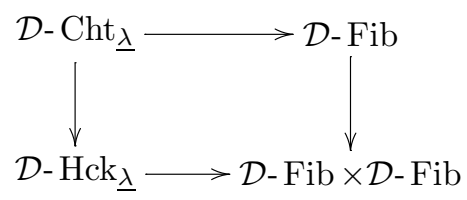

où la flèche horizontale du bas envoie la donnée de modifications itérées $\left(\left(u_{j}\right)_{j=1}^{n},\left(\mathcal{V}_{j}\right)_{j=0}^{n}\right.$, $\left.\left(t_{j}\right)_{j=1}^{n}\right)$ sur $\left(\mathcal{V}_{0}, \mathcal{V}_{n}\right)$ et la flèche verticale de droite envoie $\mathcal{V}$ sur $\left({ }^{\sigma} \mathcal{V}, \mathcal{V}\right)$. Puisque fibres par fibres au-dessus de $S,{ }^{\sigma} \mathcal{V}$ et $\mathcal{V}$ sont les fibrés sur $X$ ayant le même degré, pour que $\mathcal{D}$ - $\mathrm{Cht}_{\underline{\lambda}}$ ne soit pas vide, il est nécessaire que $\sum_{i=1}^{n} \sum_{j=1}^{d} \lambda_{i}^{j}=0$.

On peut expliciter la définition de $\mathcal{D}$ - $\mathrm{Cht}_{\underline{\lambda}}$ comme suit.

DÉFINITION 1. - Le champ $\mathcal{D}$ - $\mathrm{Cht}_{\lambda}$ associe à tout $\mathbb{F}_{q}$-schéma la catégorie en groupoïdes des données $\left(\left(u_{j}\right)_{j=1}^{n},\left(\mathcal{V}_{j}\right)_{j=0}^{n},\left(t_{j}\right)_{j=1}^{n}, \varepsilon\right)^{-}$comprenant :

- $n$ points $u_{1}, \ldots, u_{n}$ de $X^{\prime}$ à valeurs dans $S$,

- $n+1$ points $\mathcal{V}_{0}, \ldots, \mathcal{V}_{n}$ de $\mathcal{D}$ - Fib à valeurs dans $S$,

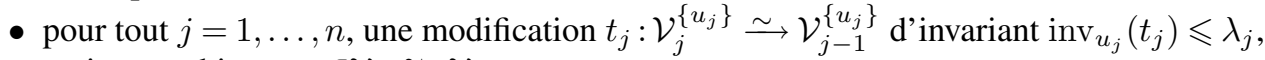

- un isomorphisme $\varepsilon:{ }^{\sigma} \mathcal{V}_{0} \stackrel{\sim}{\longrightarrow} \mathcal{V}_{n}$.

Selon une suggestion de V. Lafforgue, on peut appeler ces objets les chtoucas à mille pattes. Dans le cas particulier des chtoucas à deux pattes, et pour

$$
\lambda_{1}=\mu=(1,0, \ldots, 0) \quad \text { et } \quad \lambda_{2}=\mu^{\vee}=(0, \ldots, 0,-1)
$$

on retrouve bien la notion de $\mathcal{D}$-chtoucas usuels de rang 1 tels qu'ils sont étudiés dans [14, chapitre 4].

Si les points $u_{1}, \ldots, u_{n} \in X^{\prime}(k)$ sont deux à deux distincts, la liste des données constituantes d'un $\mathcal{D}$-chtouca, ayant ses modifications en $u_{1}, \ldots, u_{n}$, peut se réduire à une seule modification

$$
t:\left.\left.{ }^{\sigma} \mathcal{V}_{0}\right|_{X-\left\{u_{1}, \ldots, u_{n}\right\}} \stackrel{\sim}{\longrightarrow} \mathcal{V}_{0}\right|_{X-\left\{u_{1}, \ldots, u_{n}\right\}} .
$$

En effet la «grande» modification $t=\varepsilon \circ t_{n} \circ \cdots \circ t_{1}$ détermine alors chacune des composantes $\varepsilon, t_{n}, \ldots, t_{1}$ d'après la proposition 3 de 1.2 .

$\mathcal{D}$ - Cht $_{\lambda}$ a un nombre infini de composantes connexes paramétrées par le degré de $\mathcal{V}_{0}$. Soit $a \in \mathbb{A}_{F}^{\times}$un idèle de degré non nul. On pose

$$
\mathcal{S}_{\underline{\lambda}}:=\mathcal{D}-\mathrm{Cht}_{\underline{\lambda}} / a^{\mathbb{Z}}
$$

où le quotient par $a^{\mathbb{Z}}$ consiste à ajouter formellement dans la catégorie $\mathcal{D}$ - $\operatorname{Cht}_{\underline{\underline{\lambda}}}(S)$ un isomorphisme entre tout objet avec l'objet qui s'en déduit par tensorisation avec le fibré en droite $\mathcal{L}(a)$ associé à l'idèle $a$. Le morphisme évident $\mathcal{D}$ - $\mathrm{Cht}_{\underline{\lambda}} \rightarrow X^{\prime n}$ induit un morphisme

$$
c_{\underline{\lambda}}: \mathcal{S}_{\underline{\lambda}} \rightarrow X^{\prime n}
$$

qu'on appellera le morphisme caractéristique.

\subsection{Modèles locaux}

Le champ des chtoucas usuels $\mathcal{D}$ - Cht $_{\mu, \mu^{\vee}}$ est lisse au-dessus de $X^{\prime} \times X^{\prime}$. Ce ne sera plus le cas pour $\mathcal{D}$ - Cht $_{\underline{\lambda}}$ pour les suites $\underline{\lambda}=\left(\lambda_{1}, \ldots, \lambda_{n}\right)$ dont, au moins, une des composantes n'est 
pas minuscule. On peut toutefois montrer que toutes les singularités de $\mathcal{D}$ - $\mathrm{Cht}_{\underline{\lambda}}$ apparaissent déjà dans le $\mathcal{Q}_{\underline{\lambda}}$ de sorte qu'on peut l'appeler le modèle local de $\mathcal{D}$ - Cht $t_{\underline{\lambda}}$ par analogie avec les modèles locaux de variétés de Shimura avec mauvaise réduction. La théorie de déformation de variétés abéliennes de Grothendieck-Messing est ici remplacée par un lemme de type transversalité du graphe de l'endomorphisme de Frobenius avec la diagonale, dont voici l'énoncé précis, voir [14, section I.2, proposition 1].

LEMmE 1.-Considérons un diagramme de champs sur $\mathbb{F}_{q}$

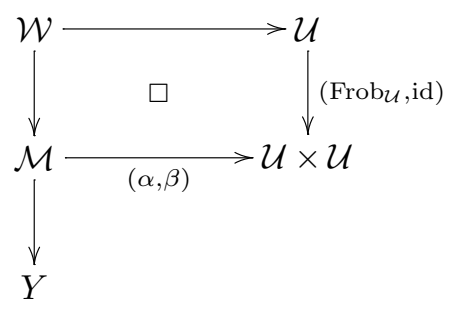

où $Y$ est un $\mathbb{F}_{q}$-schéma, où $\mathcal{U}$ est algébrique et localement de type fini sur $\mathbb{F}_{q}$, $\mathcal{M}$ est algébrique et localement de type fini sur $Y$, le morphisme $(\pi, \alpha): \mathcal{M} \rightarrow Y \times \mathcal{U}$ est représentable et où le carré est 2-cartésien.

Alors $\mathcal{W}$ est algébrique et localement de type fini sur $Y$ et le morphisme diagonal $\mathcal{W} \rightarrow$ $\mathcal{W} \times \mathcal{W}$, qui est représentable, séparé et de type fini, est partout non-ramifié et en particulier, quasi-fini.

Si on suppose de plus que $\mathcal{U}$ est lisse sur $\mathbb{F}_{q}$ et que le morphisme

$$
(\pi, \alpha): \mathcal{M} \rightarrow Y \times \mathcal{U}
$$

est lisse et purement de dimension relative $n$, alors le morphisme $\mathcal{W} \rightarrow Y$ est aussi lisse et purement de dimension relative $n$.

Soit $\underline{\lambda}=\left(\lambda_{1}, \ldots, \lambda_{n}\right) \in\left(\mathbb{Z}_{+}^{d}\right)^{n}$. En appliquant ce lemme à notre situation où $Y=\mathcal{Q}_{\underline{\lambda}}$, $\mathcal{U}=\mathcal{D}$-Fib, et où $\mathcal{M}=\mathcal{D}-\mathrm{Hck}_{\underline{\lambda}}$, on trouve que le champ $\mathcal{D}$ - $\mathrm{Cht}_{\underline{\lambda}}$ est algébrique, localement de type fini et que le morphisme

$$
\mathcal{D}-\mathrm{Cht}_{\underline{\lambda}} \rightarrow \mathcal{Q}_{\underline{\lambda}}
$$

est un morphisme lisse. On en déduit un morphisme lisse

$$
f_{\underline{\lambda}}: \mathcal{S}_{\underline{\lambda}} \rightarrow \mathcal{Q}_{\underline{\lambda}}
$$

L'image inverse $\mathcal{F}_{\underline{\lambda}}=f_{\underline{\lambda}}^{*} \mathcal{A}_{\underline{\lambda}}$ est alors le complexe d'intersection de $\mathcal{S}_{\underline{\lambda}}$.

COROLlaire 2. - Le complexe d'intersection $\mathcal{S}_{\underline{\lambda}}$ de $\mathcal{D}$ - $\mathrm{Cht}_{\underline{\lambda}}$ a les propriétés suivantes :

(1) Il est localement acyclique par rapport au morphisme caractéristique $c_{\underline{\lambda}}: \mathcal{S}_{\underline{\lambda}} \rightarrow X^{\prime n}$.

(2) Ses restrictions aux fibres de ce morphisme sont, à décalage près, des faisceaux pervers irréductibles.

\subsection{Structure de niveau}

Soit $I$ un sous-schéma fermé de $X$. On note $\mathcal{D}_{I}$ la restriction de $\mathcal{D}$ à $I$.

Soit $\widetilde{\mathcal{V}}=\left(\left(u_{i}\right),\left(\mathcal{V}_{i}\right),\left(t_{i}\right), \varepsilon\right)$ un $\mathcal{D}$-chtouca à valeur dans $S$ avec modification d'invariants bornés par $\lambda_{1}, \ldots, \lambda_{n}$ en $u_{1}, \ldots, u_{n} \in X^{\prime}(S)$, ou autrement dit, un point de $\mathcal{D}$ - Cht $_{\underline{\lambda}}$ à valeur 
dans $S$. Supposons en plus que $u_{1}, \ldots, u_{n}$ évitent $I$ c'est-à-dire $u_{1}, \ldots, u_{n} \in\left(X^{\prime}-I\right)(S)$. On peut alors définir ce qu'est une $I$-structure de niveau de $\widetilde{\mathcal{V}}$.

Puisque les $u_{i}$ évitent $I$, les modifications $t_{i}$ définissent des isomorphismes entre les restrictions des $\mathcal{V}_{i}$ à $I \times S$ qu'on notera simplement $\mathcal{V}_{I}$; c'est un $\mathcal{D}_{I} \otimes \mathcal{O}_{S}$-module localement libre de rang 1. L'isomorphisme $\varepsilon:{ }^{\sigma} \mathcal{V}_{0} \stackrel{\sim}{\longrightarrow} \mathcal{V}_{n}$ induit un isomorphisme $\varepsilon_{I}:{ }^{\sigma} \mathcal{V}_{I} \sim \mathcal{V}_{I}$.

DÉfinition 1.- Une $I$-structure de niveau de $\widetilde{\mathcal{V}}$ est la donnée d'un isomorphisme de $\mathcal{D}_{I} \otimes \mathcal{O}_{S}$-modules

$$
\iota: \mathcal{D}_{I} \otimes \mathcal{O}_{S} \rightarrow \mathcal{V}_{I}
$$

tel que le diagramme

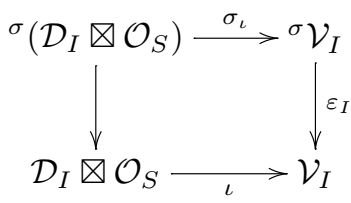

est commutatif. Ici, l'isomorphisme vertical gauche est celui qui se déduit de l'isomorphisme canonique Frob $_{S}^{*} \mathcal{O}_{S} \stackrel{\sim}{\longrightarrow} \mathcal{O}_{S}$.

On utilise une notation un peu abusive $\mathcal{D}^{I}-\mathrm{Cht}_{\underline{\lambda}}$ pour désigner le champ des $\mathcal{D}$-chtoucas de modification en dehors de $I$ de position bornée par $\underline{\lambda}$, munis d'une $I$-structure de niveau. Suivant Lafforgue, on a un diagramme 2-cartésien

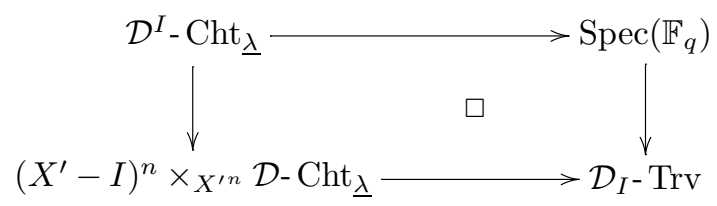

où $\mathcal{D}_{I^{-}}$Trv est le champ en groupoïdes qui associe à chaque $\mathbb{F}_{q}$-schéma $S$ la catégorie des $\mathcal{D}_{I} \otimes \mathcal{O}_{S}$-modules $\mathcal{F}$ localement libres de rang 1 munie d'un isomorphisme $\phi:{ }^{\sigma} \mathcal{F} \stackrel{\sim}{\longrightarrow} \mathcal{F}$. La flèche horizontale en bas est définie par

$$
\widetilde{\mathcal{V}} \mapsto\left(\varepsilon_{I}:{ }^{\sigma} \mathcal{V}_{I} \stackrel{\sim}{\longrightarrow} \mathcal{V}_{I}\right)
$$

La flèche verticale à droite est donnée par l'objet évident $\mathcal{F}=\mathcal{D}_{I}$ sur $\operatorname{Spec}\left(\mathbb{F}_{q}\right)$. Rappelons le théorème 2 de [14, section I.3].

PROposition 2. - La flèche verticale de droite dans le carré ci-dessus est un $\mathcal{D}_{I}^{\times}$-torseur par rapport à l'action triviale de $\mathcal{D}_{I}^{\times}$sur $\operatorname{Spec}\left(\mathbb{F}_{q}\right)$. La flèche verticale de gauche du carré est un morphisme représentable fini étale et galoisien de groupe de Galois $\mathcal{D}_{I}^{\times}$.

Posons $\mathcal{S}_{\underline{\lambda}}^{I}:=\mathcal{D}$ - $\mathrm{Cht}_{\underline{\lambda}}^{I} / a^{\mathbb{Z}}$. On obtient par composition un morphisme lisse $f_{\underline{\lambda}}^{I}: \mathcal{S}_{\underline{\lambda}}^{I} \rightarrow \mathcal{Q}_{\underline{\lambda}}$ et un morphisme

$$
c_{\underline{\lambda}}^{I}: \mathcal{S}_{\underline{\lambda}}^{I} \rightarrow\left(X^{\prime}-I\right)^{n}
$$

qu'on appelle le morphisme caractéristique de $\mathcal{S}_{\underline{\lambda}}$. Nous notons $\mathcal{F}_{\underline{\lambda}}^{I}$ l'image réciproque $\left(f_{\underline{\lambda}}^{I}\right)^{*} \mathcal{A}_{\underline{\lambda}}$.

COROLlaire 3. - Le complexe d'intersection $\mathcal{F}_{\underline{\lambda}}^{I}$ de $\mathcal{S}_{\underline{\lambda}}$ a les propriétés suivantes.

(1) Il est localement acyclique par rapport au morphisme caractéristique $c_{\underline{\lambda}}^{I}: \mathcal{S}_{\underline{\lambda}}^{I} \rightarrow$ $\left(X^{\prime}-I\right)^{n}$. 
(2) Ses restrictions aux fibres de ce morphisme sont, à décalage près, des faisceaux pervers irréductibles.

Soit $v$ un point fermé de $I$ et soit $K_{v}^{I}$ le sous-groupe compact de $G\left(F_{v}\right)$ défini comme le noyau de l'homomorphisme naturel $\left[G\left(\mathcal{O}_{v}\right) \rightarrow G\left(\mathcal{O}_{I}\right)\right]$. La donnée de $K_{v}^{I}$ définit un schéma en groupe lisse $\mathcal{G}_{v}^{I}$ sur $\mathcal{O}_{v}$ de fibres connexes, de fibre générique $G_{F_{v}}$ et dont les points entiers sont

$$
\mathcal{G}_{v}^{I}\left(\mathcal{O}_{v}\right)=K_{v}^{I}
$$

Soit $\widetilde{V}$ un $\mathcal{D}$-chtouca avec une $I$-structure de niveau défini sur $\bar{k}$. La structure de niveau induit un $\mathcal{G}_{v}^{I} \widehat{\otimes}_{\mathbb{F}_{q}} k$-torseur au-dessus $\mathcal{O}_{v} \widehat{\otimes}_{\mathbb{F}_{q}} k$. Sous l'hypothèse que les pôles et zéros du chtouca évitent $I$, l'isomorphisme $t:{ }^{\sigma} \mathcal{V}^{T} \stackrel{\sim}{\longrightarrow} \mathcal{V}$ permet de descendre le $\mathcal{G}_{v}^{I} \widehat{\otimes}_{\mathbb{F}_{q}} k$-torseur en un $\mathcal{G}_{v}^{I}$ torseur $\operatorname{sur} \mathcal{O}_{v}$ puisque $\mathcal{G}_{v}^{I}$ est un schéma en groupes de fibres connexes.

\subsection{Sur la propreté du morphisme caractéristique}

Comme nous a fait remarquer E. Lau, le morphisme caractéristique

$$
c_{\underline{\lambda}}^{I}: \mathcal{S}_{\underline{\lambda}}^{I} \rightarrow\left(X^{\prime}-I\right)^{n}
$$

n'est pas propre en général. Il est néanmoins propre si on suppose en plus que l'algèbre à division $\mathcal{D}$ a un nombre de places totalement ramifiées plus grand que le nombre de dégénérateurs. Dans cette section, on va préciser cet énoncé.

Notons toutefois que l'argument principal de l'article ne nécessite pas la propriété mais seulement de l'acyclicité locale de la cohomologie. Il est donc probable qu'il puisse être utilisé aussi dans des situations non propres du moment qu'on peut construire une bonne compactification analogue à celle de Lafforgue dans le cas $\mathrm{GL}_{n}$. Pour le lemme fondamental pour le changement de base pour $\mathrm{GL}_{n}$, on peut se restreindre aux cas des algèbres à division ayant des places totalement ramifiées en quantité.

Tout élément $\lambda \in \mathbb{Z}_{+}^{d}$ peut être écrit de façon unique sous la forme

$$
\lambda=\lambda^{+}+\lambda^{-}
$$

où $\lambda_{+} \in \mathbb{N}_{+}^{d}$ et $\lambda_{-} \in(-\mathbb{N})_{+}^{d}$ c'est-à-dire

$$
\lambda^{+}=\left(\lambda_{+}^{1} \geqslant \cdots \geqslant \lambda_{+}^{d} \geqslant 0\right)
$$

et

$$
\lambda_{-}=\left(0 \geqslant \lambda_{-}^{1} \geqslant \cdots \geqslant \lambda_{-}^{d}\right)
$$

telle que pour tous $i \in\{1, \ldots, d\}$, on a $\lambda^{i}$ est ou bien égal à $\lambda_{+}^{i}$ ou bien égal à $\lambda_{-}^{i}$. On note alors

$$
\|\lambda\|:=\max \left(\left|\lambda_{+}\right|,\left|\lambda_{-}\right|\right) .
$$

Lemme 1. - Soient $\mathcal{V}$ et $\mathcal{V}^{\prime}$ deux fibrés vectoriels de rang $d$ sur $\bar{X}_{u}$ le complété de $\bar{X}$ en un point $u \in \bar{X}$ et $t: \mathcal{V}^{u} \stackrel{\sim}{\longrightarrow} \mathcal{V}^{\prime u}$ une modification d'invariant $\operatorname{inv}_{u}(t) \leqslant \lambda$. Alors $t$ se prolonge en un morphisme de $\mathcal{O}_{\bar{X}_{u}}$-modules

$$
t: \mathcal{V} \rightarrow \mathcal{V}^{\prime}(\|\lambda\| u)
$$

$4^{\mathrm{e}}$ SÉRIE - TOME $39-2006-\mathrm{N}^{\circ} 2$ 
Démonstration. - C'est la formule d'inversion des matrices de Cramer.

Proposition 2. - Soit $\underline{\lambda}=\left(\lambda_{1}, \ldots, \lambda_{n}\right)$ avec $\lambda_{i} \in \mathbb{Z}_{+}^{d}$. Supposons que le nombre de places totalement ramifiées de $\mathcal{D}$ est supérieur ou égal à

$$
d^{2}\left(\left\|\lambda_{1}\right\|+\cdots+\left\|\lambda_{n}\right\|\right) .
$$

Alors, le morphisme $c_{\underline{\lambda}}^{I}: \mathcal{S}_{\underline{\lambda}}^{I} \rightarrow\left(X^{\prime}-I\right)^{n}$ est un morphisme propre.

Démonstration. - Nous allons reprendre l'argument de [14, IV.1] avec des modifications appropriées.

Un $\mathcal{D}$-chtouca de rang 1 est automatiquement irréductible est donc $\alpha$-semi-stable pour tout $\alpha \in] 0,1\left[\right.$ de sorte que le champ $\mathcal{D}^{I}$ - Cht $t_{\underline{\lambda}}$ est de type fini, voir [14, II.2, théorème 8]. Il nous suffira donc pour démontrer la propreté de $c_{\underline{\lambda}}^{I}$ de vérifier le critère valuatif de propreté pour ce morphisme.

Soient $\mathcal{O}$ un anneau de valuation discrète contenant $\mathbb{F}_{q}, K$ son corps des fractions, $\kappa$ son corps résiduel. Notons $\mathcal{X}=X \otimes_{\mathbb{F}_{q}} \mathcal{O}$ qu'on voit comme une courbe fibrée sur $\operatorname{Spec}(\mathcal{O})$. Notons $\eta_{\kappa}$ le point générique de la fibre spéciale $X \otimes_{\mathbb{F}_{q}} \kappa$ de $\mathcal{X}$. Notons $\mathcal{O}^{\prime}$ l'anneau local de $\mathcal{X}$ en le point $\eta_{\kappa}, K^{\prime}$ son corps des fractions, $\kappa^{\prime}$ est son corps résiduel.

Donnons-nous $n$ morphismes zéros $u_{i} \in\left(X^{\prime}-I\right)(\mathcal{O})$ avec $i=1, \ldots, n$. Pour alléger les notations, nous allons noter par les mêmes notations $u_{i}$ les $K$-points de $X^{\prime}-I$ qui s'en déduisent par extension des scalaires de $\mathcal{O}$ à $K$.

Donnons-nous un point

$$
\widetilde{\mathcal{V}}^{K}=\left(\left(u_{j}\right)_{j=1}^{n},\left(\mathcal{V}_{j}^{K}\right)_{j=0}^{n},\left(t_{j}\right)_{j=1}^{n}, \varepsilon\right)
$$

de $\mathcal{D}^{I}$ - Cht $\underline{\lambda}$ à valeurs dans $K$ au-dessus de $\left(u_{j}\right)_{j=1}^{n} \in\left(X^{\prime}-I\right)^{n}(K)$. On doit montrer qu'il peut s'étendre en un point de $\mathcal{D}^{I}$ - $\mathrm{Cht}_{\underline{\lambda}}$ à valeur dans $\mathcal{O}$ au-dessus de $\left(u_{j}\right)_{j=1}^{n} \in\left(X^{\prime}-I\right)(\mathcal{O})$. On va utiliser le lemme suivant qui est la conjonction de [24, Proposition 7] et [8, 9.4.3].

Lemme 3. - Soient $\mathcal{X}$ un schéma régulier de dimension 2 et $j: U \hookrightarrow \mathcal{X}$ une immersion ouverte dont le fermé complémentaire est de dimension zéro. Alors les foncteurs $j_{*}$ et $j^{*}$ entre la catégorie des $\mathcal{O}_{\mathcal{X}}$-modules localement libres et la catégorie des $\mathcal{O}_{U}$-modules modules localement libres sont quasi-inverses l'une de l'autre et définissent une équivalence.

Prolonger un fibré vectoriel $\mathcal{V}_{j}^{K}$ sur $X \otimes_{\mathbb{F}_{q}} K$ consiste donc à prolonger sa fibre générique $V_{i}$, de $\operatorname{Spec}\left(K^{\prime}\right)$ à $\operatorname{Spec}\left(\mathcal{O}^{\prime}\right)$, autrement dit, à choisir un $\mathcal{O}^{\prime}$-réseau dans le $K^{\prime}$-espace vectoriel $V_{j}$. Dans le $\mathcal{D}$-chtouca $\widetilde{V}^{K}$, les fibres génériques $V_{j}$ de $\mathcal{V}_{j}^{K}$ peuvent être identifiées à l'aide des modifications $t_{j}$ et nous allons les noter tous $V$. L'isomorphisme $\varepsilon:{ }^{\sigma} \mathcal{V}_{0}^{K} \stackrel{\sim}{\longrightarrow} \mathcal{V}_{0}^{\prime K}$ induit une application $\sigma$-linéaire injective $\varepsilon_{V}: V \rightarrow V$ c'est-à-dire une application $\varepsilon_{V}$ vérifiant $\varepsilon_{V}(\alpha v)=$ $\sigma(\alpha) v$ pour tout $\alpha \in K^{\prime}=F \otimes_{\mathbb{F}_{q}} K$ où $\sigma$ est défini par $\sigma(a \otimes b)=a \otimes b^{q}$ pour tout $a$ dans le corps des fonctions $F$ de $X$ et $b \in K$.

Rappelons le lemme suivant de Drinfeld [5].

LEMME 4. - Soit $V$ un $K^{\prime}$-espace vectoriel muni d'une application $\sigma$-linéaire injective $\varepsilon_{V}: V \rightarrow V$. Il existe alors des $\mathcal{O}^{\prime}$-réseaux stables sous $\varepsilon_{V}$ et parmi ceux-ci il en existe un qui contient tous les autres.

Soit $\pi$ un uniformisant de l'anneau local $\mathcal{O}$ qu'on utilise aussi comme un uniformisant de $\mathcal{O}^{\prime}$. Soient $V$ un $K^{\prime}$-espace vectoriel muni d'une application $\sigma$-linéaire injective $\varepsilon_{V}: V \rightarrow V$. Soit 
$M$ un $\mathcal{O}^{\prime}$-réseau $\varepsilon_{V}$-stable de $V$. Alors $M$ est dit admissible si l'application induite

$$
\bar{\varepsilon}_{V}: M / \pi M \rightarrow M / \pi M
$$

n'est pas nilpotente.

Rappelons un autre lemme de Drinfeld [5].

Lemme 5. - Gardons les notations du lemme précédent et notons $M_{0}$ le $\mathcal{O}^{\prime}$-réseau $\varepsilon_{V}$-stable maximal de $V$. Alors, s'il existe un seul $\mathcal{O}^{\prime}$-réseau $\varepsilon_{V}$-stable admissible dans $V$, le réseau $M_{0}$ est aussi admissible. De plus, quitte à remplacer $K$ par une extension finie $L,\left(V, \varepsilon_{V}\right)$ par $\left(V \otimes_{K} L, \varepsilon_{V} \otimes \sigma_{L}\right), V$ contiendra un réseau $t_{V}$-stable admissible.

Revenons à notre situation où $V$ est la fibre générique des composantes $\mathcal{V}_{i}^{K}$ du $\mathcal{D}$-chtouca $\widetilde{\mathcal{V}}^{K}$. Quitte à remplacer $K$ par une extension finie, on peut supposer que le $\mathcal{O}^{\prime}$-réseau $M_{0} t_{V^{-}}$ stable maximal est admissible. En utilisant $M_{0}$, on étend alors canoniquement chaque $\mathcal{V}_{K}^{j}$ en un fibré $\mathcal{V}_{j}$ sur $\mathcal{X}$. Par fonctorialité, chaque modification $t_{j}$ s'étend en un isomorphisme entre les restrictions de $\mathcal{V}_{j-1}$ et $\mathcal{V}_{j}$ en dehors du graphe de $u_{j}$. De même, les actions de la $\mathcal{O}_{X}$-algèbre $\mathcal{D}$ sur $\mathcal{V}_{j}^{K}$ et $\mathcal{V}_{I}^{\prime K}$ s'étendent en une action $\mathcal{D}$ sur $\mathcal{V}_{j}$ et $\mathcal{V}_{j}^{\prime}$. Enfin, l'isomorphisme $\varepsilon:{ }^{\sigma} \mathcal{V}_{0}^{K} \rightarrow \mathcal{V}_{n}^{K}$ s'étend en un morphisme $\varepsilon:{ }^{\sigma} \mathcal{V}_{0} \rightarrow \mathcal{V}_{n}$. Il reste à vérifier les trois assertions suivantes :

PROPOSITION 6. -

(1) Les $t_{j}: \mathcal{V}_{j-1}^{\left\{u_{j}\right\}} \rightarrow \mathcal{V}_{j}^{\left\{u_{j}\right\}}$ sont des modifications d'invariant $\operatorname{inv}_{u_{j}}\left(t_{j}\right) \leqslant \lambda^{j}$.

(2) $\varepsilon:{ }^{\sigma} \mathcal{V}_{0} \rightarrow \mathcal{V}_{n}$ est un isomorphisme.

(3) Les $\mathcal{D} \otimes \mathcal{O}_{\mathcal{X}}$-modules $\mathcal{V}_{j}$ sont localement libres.

Démonstration. - La première assertion est évidente. En effet $t_{i}$ est une modification d'invariant au plus $\lambda_{i}$ en fibre générique, il en est de même en fibre spéciale.

Vérifions la seconde assertion. D'après le lemme 3, il suffit de démontrer que $\varepsilon$ induit un isomorphisme entre les fibres de ${ }^{\sigma} \mathcal{V}_{0}$ et de $\mathcal{V}_{n}$ au point générique $\eta_{\kappa}$ de la fibre spéciale de $\mathcal{X}$. Bien entendu, il revient au même de démontrer que l'application $\bar{\varepsilon}: \bar{M}_{0} \otimes_{\kappa^{\prime}, \sigma} \kappa^{\prime} \rightarrow \bar{M}_{0}$ est un isomorphisme.

Par hypothèse d'admissibilité, l'application linéaire

$$
\bar{\varepsilon}: \bar{M}_{0} \otimes_{\kappa^{\prime}, \sigma} \kappa^{\prime} \rightarrow \bar{M}_{0}
$$

n'est pas nilpotente. Considérons la filtration de $\bar{M}_{0}$ définie par les images des puissances de $\bar{\varepsilon}$. Celle-ci est nécessairement stationnaire puisqu'il s'agit d'une filtration d'un espace vectoriel de dimension finie. Il existe donc un sous-espace vectoriel non nul $N$ de $\bar{M}_{0}$ qui est égal à l'image de $\bar{\varepsilon}^{n}$ pour tous $n$ assez grand. Supposons que $\bar{\varepsilon}$ n'est pas un isomorphisme, $N$ est alors un sous- $\kappa^{\prime}$-espace vectoriel non nul et strict de $\bar{M}_{0}$.

Par construction, l'application linéaire restreinte $\bar{\varepsilon}: N \otimes_{\kappa^{\prime}, \sigma} \kappa^{\prime} \rightarrow N$ est surjective donc bijective. Par hypothèse $\bar{\varepsilon}$ commute à l'action de $D$ de sorte que le sous-espace vectoriel $N$ est $D$-stable. Pour tout $j=0, \ldots, n$, le sous-espace vectoriel $N$ définit par saturation un sousfibré $\overline{\mathcal{V}}_{j}^{N}$ de $\overline{\mathcal{V}}_{j}=\mathcal{V}_{j} \otimes_{\mathcal{O}} \kappa$. Puisque $N$ est stable sous l'action de $D$, les saturés $\overline{\mathcal{V}}_{j}^{N}$ sont des $\mathcal{D}_{X} \otimes \mathcal{O}_{S}$-modules.

Les modifications $t_{j}: \mathcal{V}_{j-1}^{\left\{u_{j}\right\}} \rightarrow \overline{\mathcal{V}}_{j}^{\left\{u_{j}\right\}}$ d'invariants au plus $\lambda_{j}$ induisent des morphismes injectifs de $\mathcal{O}_{X}$-modules

$$
\mathcal{V}_{j} \rightarrow \mathcal{V}_{j-1}\left(\left\|\lambda_{j}\right\| \bar{u}_{j}\right)
$$

$4^{\text {e }}$ SÉRIE - TOME $39-2006-\mathrm{N}^{\circ} 2$ 
Par $S$-platitude, ceci induit un morphisme injectif sur la fibre spéciale $\mathcal{V}_{n} \rightarrow \mathcal{V}_{0}\left(\sum_{j=1}^{n}\left\|\lambda_{j}\right\| \bar{u}_{j}\right)$ et donc un morphisme injectif

$$
\overline{\mathcal{V}}_{n} \rightarrow \overline{\mathcal{V}}_{0}\left(\sum_{j=1}^{n}\left\|\lambda_{j}\right\| \bar{u}_{j}\right) .
$$

Par restriction à $N$, on a un morphisme injectif

$$
\overline{\mathcal{V}}_{n}^{N} \rightarrow \overline{\mathcal{V}}_{0}^{N}\left(\sum_{j=1}^{n}\left\|\lambda_{j}\right\| \bar{u}_{j}\right) .
$$

On en déduit l'inégalité

$$
\operatorname{deg}\left(\overline{\mathcal{V}}_{n}^{N}\right) \leqslant \operatorname{deg}\left(\overline{\mathcal{V}}_{0}^{N}\right)+d^{2} \sum_{j=1}^{N}\|\lambda\| .
$$

Par ailleurs, on a un morphisme ${ }^{\sigma} \mathcal{V}_{0} \rightarrow \mathcal{V}_{N}$ qui par restriction à la fibre spéciale, puis à $N$, induit un homomorphisme

$$
\sigma \overline{\mathcal{V}}_{0}^{N} \rightarrow \overline{\mathcal{V}}_{n}^{N}
$$

Cet homomorphisme est aussi injectif puisqu'il l'est génériquement par la construction de $N$. L'inégalité de degrés obtenue plus haut implique que son conoyau est de longueur au plus $d^{2} \sum_{j=1}^{N}\left\|\lambda_{j}\right\|$. Ce conoyau supporté par un sous-schéma fini de $X$ est appelé le dégénérateur de Drinfeld.

Par hypothèse sur le nombre de places totalement ramifiées de $D$, il existe une place $v$ de $X-X^{\prime}$ qui évite le dégénérateur où $D$ est totalement ramifiée. Il évite aussi les points $u_{1}, \ldots, u_{n} \in X^{\prime}$. On a alors un isomorphisme

$$
\bar{\varepsilon}:{ }^{\sigma} \overline{\mathcal{V}}_{0, v}^{N} \rightarrow \overline{\mathcal{V}}_{0, v}^{N}
$$

de $\mathcal{D}_{v} \otimes_{\mathbb{F}_{q}} \kappa$-modules. En prenant les vecteurs fixes par cet isomorphisme on a un $D_{v}$-sousmodule $\operatorname{Ker}\left[\bar{\varepsilon}-1, \overline{\mathcal{V}}_{0, v}^{N}\right]$ de dimension sur $F_{v}$ égal à la dimension de $N$ sur $F \otimes_{\mathbb{F}_{q}} \kappa$, en particulier strictement plus petite que $d^{2}$. Ceci n'est pas possible puisque $D_{v}$ est une algèbre à division centrale de dimension $d^{2}$ sur son centre $F_{v}$.

On a démontré un isomorphisme ${ }^{\sigma} \mathcal{V}_{0} \stackrel{\sim}{\longrightarrow} \mathcal{V}_{n}$ sur $X \times S$. Pour prolonger la structure de $\mathcal{D} \otimes \mathcal{O}_{S}$ sur les $\mathcal{V}_{i}$, on peut maintenant invoquer [14, proposition 7, I.3] et conclure la démonstration de la proposition.

\section{Systèmes locaux fondamentaux}

On va continuer à supposer que l'algèbre à division $D$ a un nombre de places totalement ramifiées plus grand que $d^{2}\|\lambda\|$. Sous cette hypothèse, le morphisme caractéristique $c_{\lambda}^{I}$ est propre d'après 1.6.

Pour tout niveau $I$, on dispose d'un morphisme propre

$$
c_{\underline{\lambda}}^{I}: \mathcal{S}_{\underline{\lambda}}^{I} \rightarrow\left(X^{\prime}-I\right)^{n}
$$

et d'un faisceau pervers $\mathcal{F}_{\underline{\lambda}}^{I}$ sur $\mathcal{S}_{\underline{\lambda}}^{I}$ qui est localement acyclique par rapport à $c_{\underline{\lambda}}^{I}$. Les images directes $\mathrm{R}^{i}\left(c_{\underline{\lambda}}^{I}\right)_{*} \mathcal{F}_{\underline{\lambda}}^{I}$ sont donc des systèmes locaux. On va considérer le systèmé local gradué 
$\mathcal{W}_{\underline{\lambda}}^{I}$ en prenant pour sa $i$-ième composante le système local $\mathrm{R}^{i}\left(c_{\underline{\lambda}}^{I}\right)_{*} \mathcal{F}_{\underline{\lambda}}^{I}$. Cette somme directe est munie d'une action de l'algèbre de Hecke $\mathcal{H}^{I}$.

\subsection{Opérateurs de Hecke et points fixes}

On va rappeler la construction des correspondances de Hecke et leurs actions sur $\mathcal{W}_{\underline{\lambda}}^{I}$. Soit $K^{I}$ le sous-groupe compact de $G\left(\mathcal{O}_{\mathbb{A}}\right)=\prod_{v \in|X|} G\left(\mathcal{O}_{x}\right)$ défini comme le noyau de l'homomorphisme naturel $\left[G\left(\mathcal{O}_{\mathbb{A}}\right) \rightarrow G\left(\mathcal{O}_{I}\right)\right]$ qui se décompose en produit des sous-groupes compacts $K_{v}^{I}$ de $G\left(\mathcal{O}_{v}\right)$, égaux à $G\left(\mathcal{O}_{v}\right)$ pour $v \notin|I|$. Pour toutes places $v \in|X|$, notons $\mathcal{H}_{v}^{I}$ l'algèbre des fonctions $K_{v}^{I}$-bi-invariantes et à support compact sur $G\left(F_{v}\right)$ et $\mathcal{H}^{I}$ leur produit restreint.

Rappelons que $\mathcal{H}_{v}^{I}$ admet une base $\left(\phi_{\beta_{v}}\right)$ formée des fonctions caractéristiques des $K_{v}^{I}$-double classes, indexées par l'ensemble des doubles classes

$$
\beta_{v} \in K_{v}^{I} \backslash G\left(F_{v}\right) / K_{v}^{I}
$$

Lorsque $v \in\left|X^{\prime}-I\right|$, cet ensemble est en bijection canonique avec $\mathbb{Z}_{+}^{d}$.

Considérons la catégorie des triplets

$$
\left(\mathcal{V}_{v}^{I}, \mathcal{V}_{v}^{\prime I}, t\right)
$$

où $\mathcal{V}_{v}^{I}$ et $\mathcal{V}_{v}^{\prime I}$ sont des $\mathcal{G}_{v}^{I}$-torseurs sur $\mathcal{O}_{v}$ et où $t$ est un isomorphisme entre leurs restrictions à $F_{v}$. Puisque $\mathcal{G}_{v}^{I}\left(F_{v}\right)=G\left(F_{v}\right)$ et $\mathcal{G}_{v}^{I}\left(\mathcal{O}_{v}\right)=K_{v}^{I}$, l'ensemble des classes d'isomorphisme de cette catégorie est l'ensemble des doubles classes $\beta_{v} \in K_{v}^{I} \backslash G\left(F_{v}\right) / K_{v}^{I}$.

Soit $v \in|X|$ et $\beta_{v} \in K_{v}^{I} \backslash G\left(F_{v}\right) / K_{v}^{I}$ une double-classe. On va considérer $\Phi_{\beta_{v}}$ le champ qui associe à tout $\mathbb{F}_{q}$-schéma $S$ la catégorie en groupoïdes des triplets $\left(\widetilde{\mathcal{V}}, \widetilde{\mathcal{V}}^{\prime} ; t^{\prime \prime}\right)$ formés des données suivantes

- de deux $\mathcal{D}$-chtoucas

$$
\widetilde{\mathcal{V}}=\left(\left(u_{j}\right)_{j=1}^{n},\left(\mathcal{V}_{j}\right)_{j=0}^{n},\left(t_{j}\right)_{j=0}^{n}, \varepsilon, \iota\right)
$$

et

$$
\widetilde{\mathcal{V}}^{\prime}=\left(\left(u_{j}\right)_{j=1}^{n},\left(\mathcal{V}_{j}^{\prime}\right)_{j=0}^{n},\left(t_{j}^{\prime}\right)_{j=0}^{n}, \varepsilon^{\prime}, \iota\right)
$$

de modifications d'invariants $\operatorname{inv}_{u_{j}}\left(t_{j}\right) \leqslant \lambda_{j}$ et $\operatorname{inv}_{u_{j}}\left(t_{j}^{\prime}\right) \leqslant \lambda_{j}$, tous les deux munis de $I$-structure $\iota$ et $\iota^{\prime}$ respectivement, et ayant les mêmes zéros et pôles

$$
u_{1}, \ldots, u_{n} \in\left(X^{\prime}-I-\{v\}\right)(S)
$$

autrement dit c'est un $S$-point de

$$
\mathcal{D}^{I}-\mathrm{Cht}_{\underline{\lambda}} \times{ }_{\left(X^{\prime}-I\right)^{n}} \mathcal{D}^{I}-\mathrm{Cht}_{\underline{\lambda}} \times{ }_{\left(X^{\prime}-I\right)^{n}}\left(X^{\prime}-I-\{v\}\right)^{n},
$$

- d'une $\{v\}$-modification $t^{\prime \prime}: \widetilde{\mathcal{V}}^{\{v\}} \stackrel{\sim}{\longrightarrow} \widetilde{\mathcal{V}}^{\prime\{v\}}$ compatible avec $t$ et $t^{\prime}$ et qui vérifie la condition suivante. Le $\mathcal{D}$-chtouca $\widetilde{\mathcal{V}}$ muni d'une $I$-structure de niveau définit en $v$ un $\mathcal{G}_{v}^{I}$-torseur $\mathcal{V}_{v}^{I}$ sur $\mathcal{O}_{v}$ comme dans 1.5. De même, $\widetilde{\mathcal{V}}^{\prime}$ définit un $\mathcal{G}_{v}^{I}$-torseur $\mathcal{V}_{v}^{\prime I}$ sur $\mathcal{O}_{v}$. La $\{v\}$-modification $t^{\prime \prime}$ définit un isomorphisme entre les restrictions de ces deux $\mathcal{G}_{v}^{I}$-torseurs à $F_{v}$. On demande que la classe d'isomorphisme du triplet $\left(\mathcal{V}_{v}^{I}, \mathcal{V}_{v}^{\prime}, t^{\prime \prime}\right)$ soit la double-classe $\beta_{v} \in K_{v}^{I} \backslash G\left(F_{v}\right) / K_{v}^{I}$. 
PROPOSITION 1.-Les projections

$$
\operatorname{pr}_{1}, \operatorname{pr}_{2}: \Phi_{\beta_{v}} \rightarrow \mathcal{D}^{I}-\mathrm{Cht}_{\underline{\lambda}} \times_{\left(X^{\prime}-I\right)^{n}}\left(X^{\prime}-I-\{v\}\right)^{n}
$$

sont des morphismes finis et étales. De plus, on a $f_{\underline{\lambda}} \circ \mathrm{pr}_{1}=f_{\underline{\lambda}} \circ \mathrm{pr}_{2}$ où $f_{\underline{\lambda}}: \mathcal{D}^{I}-\mathrm{Cht}_{\underline{\lambda}} \rightarrow \mathcal{Q}_{\underline{\lambda}}$ est le morphisme défini en 1.4.

Démonstration. - Voir [14, I.4, proposition 3] pour la démonstration du fait que $\mathrm{pr}_{1}$ et $\mathrm{pr}_{2}$ sont finis et étales. L'égalité $f_{\underline{\lambda}} \circ \mathrm{pr}_{1}=f_{\underline{\lambda}} \circ \mathrm{pr}_{2}$ est claire sur définitions.

Par passage au quotient par l'action libre de $a^{\mathbb{Z}}$, on en déduit des morphismes finis étales

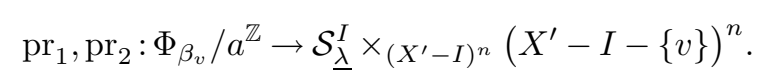

De même, on a $f_{\underline{\lambda}} \circ \operatorname{pr}_{1}=f_{\underline{\lambda}} \circ \operatorname{pr}_{2}$ où $f_{\underline{\lambda}}: \mathcal{S}_{\underline{\lambda}} \rightarrow \mathcal{Q}_{\underline{\lambda}}$ est le morphisme défini en 1.4. On en déduit un isomorphisme $\operatorname{pr}_{1}^{*} \mathcal{F}_{\underline{\lambda}} \stackrel{\sim}{\longrightarrow} \operatorname{pr}_{2}^{*} \mathcal{F}_{\underline{\lambda}}$ et donc une correspondance cohomologique de $\left(\mathcal{S}_{\underline{\lambda}}, \mathcal{F}_{\underline{\lambda}}\right)$ au-dessus de $\left(X^{\prime}-I-\{v\}\right)^{n}$. La correspondance $\Phi_{\beta_{v}}$ détermine ainsi un endomorphisme de la restriction de $\mathcal{W}_{\underline{\lambda}}^{I}$ à $\left(X^{\prime}-I-\{v\}\right)^{n}$. Puisque $\mathcal{W}_{\underline{\lambda}}^{I}$ est un système local gradué sur $\left(X^{\prime}-I\right)^{n}$, on obtient par prolongement un endomorphisme de $\mathcal{W}_{\underline{\underline{\lambda}}}^{I}$ qu'on notera $\Phi_{\beta_{v}}$ également.

Proposition 2. - Lorsque $v \in|X|$ et $\beta_{v} \in K_{v}^{I} \backslash G\left(F_{v}\right) / K_{v}^{I}$ varient, les $\Phi_{\beta_{v}}$ définissent une action de $\mathcal{H}^{I}$ sur $\mathcal{W}_{\underline{\underline{\lambda}}}^{I}$.

Démonstration. - Voir [14, 1.4, théorème 5].

\subsection{La forme conjecturale du module virtuel}

Pour tout système local $\mathcal{W} \operatorname{sur}\left(X^{\prime}-I\right)^{n}$ muni d'une action de $\mathcal{H}^{I}$, on note $[\mathcal{W}]$ sa classe dans le groupe de Grothendieck des $\mathcal{H}^{I} \otimes \pi_{1}\left(X^{\prime}-I\right)^{n}$-modules. Notons $\left[\mathcal{W}_{\underline{\lambda}}^{I}\right]$ la somme alternée

$$
\left[\mathcal{W}_{\underline{\lambda}}^{I}\right]=\sum_{i}(-1)^{i}\left[\mathcal{W}_{\underline{\lambda}, i}^{I}\right]
$$

L'expression suivante est une variante des conjectures de Langlands sur la cohomologie des variétés de Shimura. Notons l'absence des termes endoscopiques dans notre cas où le groupe $G$ est une forme intérieure de $\mathrm{GL}_{d}$.

Soient $\lambda_{1}, \ldots, \lambda_{n} \in \mathbb{Z}_{+}^{d}$ tels que $\sum_{j=1}^{n} \sum_{i=1}^{d} \lambda_{j}^{i}=0$. Alors on devrait avoir

$$
\left[\mathcal{W}_{\underline{\lambda}}^{I}\right]=\bigoplus_{\pi} m(\pi)\left[\pi^{K_{I}} \otimes \operatorname{pr}_{1}^{*} \mathcal{L}_{\lambda_{1}}(\pi) \otimes \cdots \otimes \operatorname{pr}_{n}^{*} \mathcal{L}_{\lambda_{n}}(\pi)\right]
$$

où

- $\pi$ parcourt l'ensemble des représentations automorphes de $D^{\times}$sur lesquelles $a^{\mathbb{Z}}$ agit trivialement,

- le sous-groupe compact $K_{I}$ de $G\left(\mathbb{A}_{F}\right)$ est associé au niveau $I$ de façon usuelle; $\pi^{K_{I}}$ est l'espace des vecteurs fixes de $\pi$ sous ce groupe,

- $\operatorname{pr}_{i}$ est la projection de $\left(X^{\prime}-I\right)^{n}$ sur son $i$-ième facteur,

- $\mathcal{L}_{\lambda_{i}}(\pi)$ est le système local sur $\left(X^{\prime}-I\right)$ obtenu en composant le paramètre de Langlands de $\pi$

$$
\sigma_{\pi}: \pi_{1}\left(X^{\prime}-I\right) \rightarrow \mathrm{GL}_{d}\left(\mathbb{Q}_{\ell}\right)
$$

avec la représentation algébrique irréductible de $\mathrm{GL}_{d}\left(\mathbb{Q}_{\ell}\right)$ de plus haut poids $\lambda_{i}$. 
En particulier, lorsque $n=1$, et $\lambda=\lambda_{1}$ tel que $|\lambda|=\sum_{i=1}^{d} \lambda^{i}=0$ on devrait avoir la formule simple

$$
\left[\mathcal{W}_{\lambda}^{I}\right]=\bigoplus_{\pi} m(\pi)\left[\pi^{K_{I}} \otimes \mathcal{L}_{\lambda}(\pi)\right]
$$

\section{L'action du groupe symétrique $\mathfrak{S}_{r}$}

Dans ce chapitre nous continuons à supposer que $D$ a un grand nombre de places totalement ramifiées comme dans l'énoncé de la proposition 2 de 1.6.

\subsection{Situation $\mathrm{A}$ : descente à la Weil}

Soit $\lambda \in \mathbb{Z}_{+}^{d}$ tel que $|\lambda|=0$. Pour tout entier naturel $r$, considérons les produits cartésiens $r$ fois

$$
\left(c_{\lambda}^{I}\right)^{r}:\left(\mathcal{S}_{\lambda}^{I}\right)^{r} \rightarrow\left(X^{\prime}-I\right)^{r}
$$

sur lesquels le groupe symétrique $\mathfrak{S}_{r}$ agit de manière évidente.

Notons $A$ la somme directe des images directes dérivées $\mathrm{R}^{i}\left(c_{\lambda}^{I}\right)_{*}^{r} \mathcal{F}_{\lambda}^{\bigotimes r}$ qui est $\left(\mathcal{W}_{\lambda}^{I}\right)^{\bigotimes r}$ par Künneth. Le système local gradué $A$ est muni en plus de l'action de $\left(\mathcal{H}^{I}\right)^{\otimes r}$ d'une action de $\mathfrak{S}_{r}$ qui relève de l'action de $\mathfrak{S}_{r}$ sur $\left(X^{\prime}-I\right)^{r}$.

Soient $x \in\left(X^{\prime}-I\right)(k)$ et $x^{r} \in\left(X^{\prime}-I\right)^{r}(k)$ le point diagonal dont toutes les coordonnées sont $x$. L'actions de $\mathfrak{S}_{r}$ sur $\left(X^{\prime}-I\right)^{r}$ définit alors une action de $\mathfrak{S}_{r}$ sur le groupe fondamental $\pi_{1}\left(\left(X^{\prime}-I\right)^{r}, x^{r}\right)$. Dire que l'action $\mathfrak{S}_{r}$ se relève sur $A$ revient à dire que la représentation du groupe $\pi_{1}\left(\left(X^{\prime}-I\right)^{r}, x^{r}\right)$ sur $A_{x}$ se prolonge en une représentation du produit semi-direct

$$
\pi_{1}\left(\left(X^{\prime}-I\right)^{r}, x^{r}\right) \rtimes \mathfrak{S}_{r} .
$$

Il y a une équivalence entre la catégorie des systèmes locaux sur $\left(X^{\prime}-I\right)^{r}$ munis d'une action compatible de $\mathfrak{S}_{r}$ et la catégorie des représentations du produit semi-direct $\pi_{1}\left(\left(X^{\prime}-I\right)^{r}, x^{r}\right) \rtimes$ $\mathfrak{S}_{r}$.

La fibre $A_{x^{r}}$ de $A$ en $x^{r}$ est alors un espace vectoriel gradué muni des actions compatibles de $\pi_{1}\left(\left(X^{\prime}-I\right)^{r}, x^{r}\right) \rtimes \mathfrak{S}_{r}$ et de $\left(\mathcal{H}^{I}\right)^{\otimes r}$. En admettant la formule conjecturale du paragraphe 2.3, on devrait avoir une égalité dans le groupe de Grothendieck

$$
\left[A_{x^{r}}\right]=\left[\bigoplus_{\pi_{1}, \ldots, \pi_{r}} \bigotimes_{i=1}^{r} m\left(\pi_{i}\right) \pi_{i}^{K_{I}} \otimes \bigotimes_{i=1}^{r} \mathcal{L}_{\lambda}\left(\pi_{i}\right)_{x}\right]
$$

où

- les $\pi_{1}, \ldots, \pi_{r}$ parcourent l'ensemble des représentations automorphes de $G$ sur lesquelles $a^{\mathbb{Z}}$ agit trivialement,

- $m\left(\pi_{i}\right)$ est la multiplicité de $\pi_{i}$,

- $\mathcal{L}_{\lambda}\left(\pi_{i}\right)$ est le système local sur $\left(X^{\prime}-I\right)$ associé à la représentation automorphe $\pi_{i}$ et au copoids dominant $\lambda$,

- l'action d'un élément $\tau \in \mathfrak{S}_{r}$ envoie le terme indexé par $\left(\pi_{1}, \ldots, \pi_{r}\right)$ sur le celui indexé par $\left(\pi_{\tau^{-1}(1)}, \ldots, \pi_{\tau^{-1}(r)}\right)$.

À la place de l'action de tout le groupe symétrique $\mathfrak{S}_{r}$, on n'aura besoin de regarder que l'action du sous-groupe des permutations cycliques $\mathbb{Z} / r \mathbb{Z}$ de $\mathfrak{S}_{r}$. On notera désormais $\tau$ le générateur de ce groupe cyclique. En tordant la $\mathbb{F}_{q}$-structure évidente sur $\left(X^{\prime}-I\right)^{r}$ par l'automorphisme $\tau$, on obtient la $\mathbb{F}_{q}$-structure obtenue par la descente à la Weil de $\mathbb{F}_{q^{r}}$ à $\mathbb{F}_{q}$.

$4^{\mathrm{e}}$ SÉRIE - TOME $39-2006-\mathrm{N}^{\circ} 2$ 


\subsection{Situation $B: \mathcal{D}$-chtoucas à modification symétrique}

Soit $\lambda \in \mathbb{Z}_{+}^{d}$ tel que $|\lambda|=0$. Pour tout entier naturel $r$, considérons la suite $r . \lambda$

$$
(r . \lambda):=(\underbrace{\lambda, \ldots, \lambda}_{r})
$$

Supposons que $\mathcal{D}$ a au moins $r d^{2}\|\lambda\|$ places totalement ramifiées de sorte que le morphisme

$$
c_{r . \lambda}^{I}: \mathcal{S}_{r \lambda}^{I} \rightarrow\left(X^{\prime}-I\right)^{r}
$$

est propre d'après 1.6. La propreté de $c_{r . \lambda}^{I}$ et l'acyclicité locale du complexe d'intersection $\mathcal{F}_{r . \lambda}$ impliquent que les images directes supérieures $\mathrm{R}^{i}\left(c_{r . \lambda}^{I}\right)_{*} \mathcal{F}_{r . \lambda}$ sont des systèmes locaux. Considérons le système local gradué $\mathcal{W}_{r . \lambda}^{I}$ défini en prenant la somme alternée et notons-le $B$. En plus de l'action de l'algèbre de Hecke $\mathcal{H}^{I}, B$ est muni d'une action du groupe symétrique $\mathfrak{S}_{r}$ défini comme suit.

Soit $U_{r}^{I}$ l'ouvert dans $\left(X^{\prime}-I\right)^{r}$ complémentaire de la réunion de toutes les diagonales. L'action restreinte de $\mathfrak{S}_{r}$ à $U_{r}^{I}$ est libre.

PROPOSITION 1. - L'action de $\mathfrak{S}_{r}$ sur $U_{r}^{I}$ se relève sur $\mathcal{D}$ - $\mathrm{Cht}_{r . \lambda}^{I} \times{ }_{\left(X^{\prime}-I\right)^{r}} U_{r}^{I}$.

Démonstration. - D'après la propriété de factorisation, proposition 3 de 1.2, sur l'ouvert $U_{r}^{I}$ la donnée d'un point de $\mathcal{D}$ - $\operatorname{Cht}_{r . \lambda}^{I}$ au-dessus de $u=\left(u_{i}\right)_{i=1, \ldots, r} \in U_{r}^{I}$, est la donnée d'une seule modification ${ }^{\sigma} \mathcal{V}_{0}^{T} \rightarrow \mathcal{V}_{0}^{T}$ où $T$ est la réunion des points $u_{i}$, ayant un invariant inférieur où égal à $\lambda$ en chaque point $u_{i}$. Cette description ne dépend pas de l'ordre entre les $u^{i}$. On en déduit une action du groupe symétrique $\mathfrak{S}_{r}$.

L'action de $\mathfrak{S}_{r}$ sur $\mathcal{D}$ - $\operatorname{Cht}_{r \lambda}^{I} \times\left(X^{\prime}-I\right)^{r} U_{r}^{I}$ définie ci-dessus commute de manière évidente à l'action libre de $a^{\mathbb{Z}}$. On en déduit une action de $\mathfrak{S}_{r}$ sur $\mathcal{S}_{r . \underline{\lambda}}^{I}$. Par conséquent, $\mathfrak{S}_{r}$ agit sur la restriction du système local gradué $B$ à $U_{r}^{I}$. Mais puisque c'est un système local, cette action se prolonge en une action de $\mathfrak{S}_{r}$ sur $B$ relevant l'action de $\mathfrak{S}_{r}$ sur $\left(X^{\prime}-I\right)^{r}$.

Soit $x^{r} \in\left(X^{\prime}-I\right)^{r}(k)$ le point diagonal choisi en 3.1. La fibre $B_{x^{r}}$ de $B$ en $x^{r}$ est alors munie des actions commutantes de $\pi_{1}\left(\left(X^{\prime}-I\right)^{r}, x^{r}\right) \rtimes \mathfrak{S}_{r}$ et de $\mathcal{H}^{I}$. En admettant la formule de la section 2.3, on devrait avoir une égalité dans le groupe de Grothendieck

$$
\left[B_{x}\right]=\bigoplus_{\pi} m(\pi)\left[\pi^{K_{I}} \otimes \bigotimes_{i=1}^{r} \mathcal{L}_{\lambda}(\pi)_{x}\right]
$$

où $\pi$ parcourt l'ensemble des représentations automorphes de $G$ sur lesquelles $a^{\mathbb{Z}}$ agit trivialement et où l'action de $\mathfrak{S}_{r}$ sur la somme directe devrait se déduire de l'action de $\mathfrak{S}_{r}$ par permutation sur le terme $\bigotimes_{i=1}^{r} \mathcal{L}_{\lambda}(\pi)$.

\subsection{L'argument heuristique de comparaison}

Rappelons qu'on s'est donné un élément $\lambda \in \mathbb{Z}_{+}^{d}$ tel que $|\lambda|=0$ et une algèbre à division $D$ sur $F$ ayant un nombre de places totalement ramifiées plus grand que $d^{2} r\|\lambda\|$. Cette dernière hypothèse garantit que les morphismes caractéristiques $c_{\lambda}^{I}$ et $c_{r . \lambda}^{I}$ sont propres.

Soient $A_{x^{r}}$ et $B_{x^{r}}$ les espaces vectoriels gradués, munis d'actions du groupe fondamental $\pi_{1}\left(\left(X^{\prime}-I\right)^{r}, x^{r}\right)$, de l'algèbre de Hecke $\mathcal{H}^{I}$ et du groupe symétrique, définis dans 3.1 et 3.2. 
ThÉORÈme 1. - Soient $x \in\left(X^{\prime}-I\right)(k)$ et $x^{r}$ le point diagonal de $\left(X^{\prime}-I\right)^{r}$ de coordonnées $x$. Pour tous $g \in \pi_{1}\left(\left(X^{\prime}-I\right)^{r}, x^{r}\right)$ et $f \in \mathcal{H}^{I}$ on a l'égalité

$$
\operatorname{Tr}\left((f \otimes 1 \otimes \cdots \otimes 1) g \tau, A_{x^{r}}\right)=\operatorname{Tr}\left(f g \tau, B_{x^{r}}\right)
$$

où $\tau \in \mathfrak{S}_{r}$ est la permutation cyclique.

Il nous semble utile de présenter un argument heuristique en se basant sur les expressions plausibles, voir 2.3 , de $\left[A_{x}\right]$ et de $\left[B_{x}\right]$.

Dans la somme $\left[A_{x}\right], \tau$ envoie le terme indexé par $\left(\pi_{1}, \ldots, \pi_{r}\right)$ sur le terme indexé par $\left(\pi_{r}, \pi_{1}, \ldots, \pi_{r-1}\right)$. Considérons l'ensemble des termes qui forment une orbite cyclique sous le groupe engendré par $\tau$. La somme des termes dans cette orbite est stable sous $(f \otimes 1 \otimes \cdots \otimes 1) g \tau$, mais la trace de cet opérateur sur cette somme est nulle, sauf si l'orbite est constituée d'un seul élément, c'est-à-dire $\pi_{1}=\cdots=\pi_{r}$. Pour un tel terme, diagonal, la comparaison résulte d'un lemme général d'algèbre, qui a été sans doute connu de Saito et Shintani [25].

Lemme 2. - Soit $V$ un espace vectoriel de dimension finie sur un corps K. Notons $\tau$ l'endomorphisme de $V^{\otimes r}$ défini par

$$
v_{1} \otimes \cdots \otimes v_{r} \mapsto v_{r} \otimes v_{1} \otimes \cdots \otimes v_{r-1} .
$$

Pour tous endomorphismes $f_{1}, \ldots, f_{r}$, on a

$$
\operatorname{Tr}\left(f_{1} f_{2} \ldots f_{r}, V\right)=\operatorname{Tr}\left(\left(f_{1} \otimes \cdots \otimes f_{r}\right) \tau, V^{\otimes r}\right) .
$$

Nous allons en fait démontrer directement le théorème 1 et en déduire le lemme fondamental pour le changement de base pour des fonctions sphériques de $\mathrm{GL}_{n}$ de degré 0 . La démonstration est basée sur le comptage et la théorie des modèles locaux.

\section{Comptage}

\subsection{Problème de comptage}

On va fixer une clôture algébrique $k$ de $\mathbb{F}_{q}$. Donnons-nous un entier naturel $s \geqslant 1$, et deux fermés finis disjoints $T$ et $T^{\prime}$ de $X$. Supposons de plus que $T$ évite le lieu $X-X^{\prime}$ où l'algèbre à division $D$ se ramifie.

Considérons la catégorie $\mathfrak{C}\left(T, T^{\prime} ; s\right)$ dont les objets sont des $\mathcal{D}$-fibrés $\mathcal{V}$ sur $X \otimes_{\mathbb{F}_{q}} k$ muni de deux modifications :

- une $T$-modification $t:{ }^{\sigma} \mathcal{V}^{T} \rightarrow \mathcal{V}^{T}$,

- une $T^{\prime}$-modification $t^{\prime}: \sigma^{s} \mathcal{V}^{T^{\prime}} \rightarrow \mathcal{V}^{T^{\prime}}$, telles que le diagramme

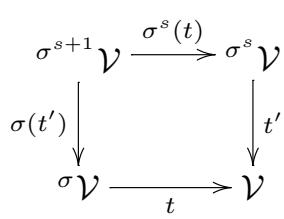

commute sur le plus grand ouvert de $\bar{X}$ où toutes les flèches sont définies.

Les morphismes de la catégorie $\mathfrak{C}\left(T, T^{\prime} ; s\right)$ sont des isomorphismes entre les triplets $\left(\mathcal{V} ; t, t^{\prime}\right)$ augmentés formellement d'un isomorphisme entre $\left(\mathcal{V} ; t, t^{\prime}\right)$ et $\left(\mathcal{V} \otimes \mathcal{L}(a) ; t \otimes \operatorname{id}_{\mathcal{L}(a)}, t^{\prime} \otimes \operatorname{id}_{\mathcal{L}(a)}\right)$

$4^{\text {e }}$ SÉRIE - TOME $39-2006-\mathrm{N}^{\circ} 2$ 
pour tous $\left(\mathcal{V} ; t, t^{\prime}\right)$. Ici, $\mathcal{L}(a)$ est le fibré en droites sur $X$ associé à l'idèle $a$ qu'on a fixé d'emblée. Un isomorphisme entre deux objets de cette catégorie sera appelé un $a$-isomorphisme.

Soit $I$ un fermé fini de $X$, étranger de $T$. Soit $K^{I}=\prod_{v \in|X|} K_{v}^{I}$ sous-groupe ouvert compact de $G\left(\mathcal{O}_{\mathbb{A}}\right)$ défini dans 2.1. Donnons-nous deux fonctions

$$
\alpha_{T}:\left|T \otimes_{\mathbb{F}_{q}} \mathbb{F}_{q^{s}}\right| \rightarrow \mathbb{Z}_{+}^{d}
$$

et $\beta_{T^{\prime}}$ qui associe à toute place $v \in\left|T^{\prime}\right|$ une double-classe

$$
\beta_{v} \in K_{v}^{I} \backslash G\left(F_{v}\right) / K_{v}^{I} .
$$

Considérons la catégorie

$$
\mathfrak{C}_{\alpha_{T}, \beta_{T^{\prime}}}^{I}\left(T, T^{\prime} ; s\right)
$$

dont les objets $\left(\mathcal{V}, t, t^{\prime}, \iota\right)$ consistent en les données suivantes

- un $T$-chtouca $(\mathcal{V}, t)$ d'invariant de Hodge $\alpha_{T}$, à valeur dans $k$ et muni d'une $I$-structure de niveau $\iota$. Ici $\alpha_{T}$ est vu comme la fonction $T \otimes_{\mathbb{F}_{q}} k$ qui se factorise par $\alpha_{T}:\left|T \otimes_{\mathbb{F}_{q}} \mathbb{F}_{q^{s}}\right| \rightarrow$ $\mathbb{Z}_{+}^{d}$,

- une $T^{\prime}$-modification $t^{\prime}: \sigma^{s} \mathcal{V}^{T^{\prime}} \stackrel{\sim}{\longrightarrow} \mathcal{V}^{T^{\prime}}$, commutant à $t$ et qui en toute place $v \in T^{\prime}$, est de type $\beta_{v}$ dans le sens de 2.1 .

Notre problème de base consiste à exprimer le nombre

$$
\# \mathfrak{C}_{\alpha_{T}, \beta_{T^{\prime}}}^{I}\left(T, T^{\prime} ; s\right):=\sum_{\left(\mathcal{V} ; t, t^{\prime} ; \iota\right)} \frac{1}{\# \operatorname{Isom}\left(\mathcal{V} ; t, t^{\prime} ; \iota\right)}
$$

où $\left(\mathcal{V} ; t, t^{\prime} ; \iota\right)$ parcourt un ensemble de représentants des classes d'isomorphisme d'objets de $\mathfrak{C}_{\alpha_{T}, \beta_{T^{\prime}}}^{I}\left(T, T^{\prime} ; s\right)$, en termes d'intégrales orbitales et d'intégrales orbitales tordues en suivant le comptage à la Kottwitz des points des variétés de Shimura.

\subsection{La fibre générique de $\left(\mathcal{V} ; t, t^{\prime}\right)$}

Soient $T, T^{\prime}$ deux sous-schémas fermés finis disjoints de $\bar{X}, T \subset \bar{X}^{\prime}$ et $s \geqslant 1$ un entier naturel comme précédemment. Soit $\left(\mathcal{V} ; t, t^{\prime}\right)$ un objet de $\mathfrak{C}\left(T, T^{\prime} ; s\right)$. Notons $V$ la fibre générique de $\mathcal{V}$; c'est un $D^{o p} \otimes_{\mathbb{F}_{q}} k$-module libre de rang 1. Les modifications $t$ et $t^{\prime}$ induisent des applications bijectives $\tau$ et $\tau^{\prime}$ de $V$ dans $V$ qui sont respectivement $\operatorname{id}_{D^{o p}} \otimes \sigma$-linéaire et $\operatorname{id}_{D^{o p}} \otimes \sigma^{s}$-linéaire. L'hypothèse de commutation entre $t$ et $t^{\prime}$ implique que $\tau \tau^{\prime}=\tau^{\prime} \tau$.

Proposition 1. - Soient $\left(\mathcal{V} ; t, t^{\prime}\right)$ un objet de $\mathfrak{C}\left(T, T^{\prime} ; s\right)$ et $\left(V ; \tau, \tau^{\prime}\right)$ sa fibre générique. Soient $v$ un point fermé de $X-T, V_{v}$ le complété de $V$ en $v, \tau_{v}: V_{v} \rightarrow V_{v}$ la bijection $\operatorname{id}_{D_{v}} \widehat{\otimes} \sigma$ linéaire induite de $\tau$. Alors, il existe un isomorphisme

$$
\left(V_{v}, \tau_{v}\right) \simeq\left(D_{v} \widehat{\otimes}_{\mathbb{F}_{q}} k, \operatorname{id}_{D_{v}} \widehat{\otimes} \sigma\right) .
$$

De même, soit v un point fermé de $X-T^{\prime}$. Alors il existe un isomorphisme

$$
\left(V_{v}, \tau_{v}^{\prime}\right) \simeq\left(\left(D_{v} \widehat{\otimes}_{\mathbb{F}_{q}} \mathbb{F}_{q^{s}}\right) \widehat{\otimes}_{\mathbb{F}_{q}^{s}} k, \mathrm{id}_{D_{v} \widehat{\otimes}_{\mathbb{F}_{q}} \mathbb{F}_{q^{s}}} \widehat{\otimes} \sigma^{s}\right)
$$

Démonstration. - Pour une place $v \notin T$, le $D_{v} \widehat{\otimes}_{\mathbb{F}_{q}} k$-module $V_{v}$ admet un $\mathcal{D}_{v} \widehat{\otimes}_{\mathbb{F}_{q}} k$-réseau $\mathcal{V}_{v}$ tel que $\tau_{v}\left(\mathcal{V}_{v}\right)=\mathcal{V}_{v}$. Il s'ensuit que $V_{v}^{\tau_{x}=1}$ est un $\mathcal{D}_{v}$-module libre de rang 1 dont le produit 
tensoriel complété avec $V_{v}^{\tau_{x}=1} \widehat{\otimes}_{\mathbb{F}_{q}} k$ est $V_{v}$. Il s'ensuit que la paire $\left(V_{v}, \tau_{v}\right)$ est isomorphe à $\left(D_{v} \widehat{\otimes}_{\mathbb{F}_{q}} k, \mathrm{id}_{D_{v}} \widehat{\otimes} \sigma\right)$. Le même argument vaut pour $T^{\prime}$.

Soit $C\left(T, T^{\prime} ; s\right)$ la catégorie dont les objets sont des triplets $\left(V ; \tau, \tau^{\prime}\right)$ où $V$ est un $D \otimes_{\mathbb{F}_{q}} k$ module libre de rang $1, \tau$ et $\tau^{\prime}$ sont des bijections $V \rightarrow V$ qui sont $\operatorname{id}_{D} \otimes \sigma$-linéaire et $\operatorname{id}_{D} \otimes \sigma^{s}$ linéaire respectivement qui vérifient les propriétés suivantes

- $\tau$ et $\tau^{\prime}$ commutent : $\tau \tau^{\prime}=\tau^{\prime} \tau$,

- pour toute place $v \notin|T|$, on a $\left(V_{v}, \tau_{v}\right)$ est isomorphe à

$$
\left(D_{v} \widehat{\otimes}_{\mathbb{F}_{q}} k, \operatorname{id}_{D_{x}} \widehat{\otimes} \sigma\right),
$$

- pour toute place $v \notin\left|T^{\prime}\right|$, on a $\left(V_{v}, \tau_{v}^{\prime}\right)$ est isomorphe à

$$
\left(\left(D_{v} \widehat{\otimes}_{\mathbb{F}_{q}} \mathbb{F}_{q^{s}}\right) \widehat{\otimes}_{\mathbb{F}_{q} s} k, \mathrm{id}_{D_{v} \widehat{\otimes}_{\mathbb{P}_{q}} \mathbb{F}_{q^{s}}} \widehat{\otimes} \sigma^{s}\right) .
$$

Les flèches de $C\left(T, T^{\prime} ; s\right)$ sont des isomorphismes entre les triplets $\left(V ; \tau, \tau^{\prime}\right)$.

Proposition 2. - Supposons que D a au moins une place totalement ramifiée. Alors tout objet $\left(V ; \tau, \tau^{\prime}\right)$ de $C\left(T, T^{\prime} ; s\right)$ est irréductible.

Démonstration. - Soit $x \in|X|$ une place où $D$ est totalement ramifiée. Puisque $x \notin T$, la paire $\left(V_{x}, \tau_{x}\right)$ est isomorphe à $\left(D_{x} \widehat{\otimes} k, \mathrm{id}_{D_{x}} \widehat{\otimes} \sigma\right)$. Cette paire est irréductible puisque $D_{x}$ est une algèbre à division centrale sur $F_{x}$.

D'après la proposition 1 , on a un foncteur de la catégorie $\mathfrak{C}\left(\bar{T}, \bar{T}^{\prime} ; s\right)$ dans la catégorie $C\left(T, T^{\prime} ; s\right)$ qui associe à un triplet $\left(\mathcal{V} ; t, t^{\prime}\right)$ sa fibre générique $\left(V ; \tau, \tau^{\prime}\right)$. Sans savoir a priori que ce foncteur est essentiellement surjectif, on va appeler $C\left(T, T^{\prime} ; s\right)$ la catégorie des fibres génériques de $\mathfrak{C}\left(\bar{T}, \bar{T}^{\prime} ; s\right)$, et chercher à classifier les classes d'isomorphisme des objets de $C\left(T, T^{\prime} ; s\right)$. Pour cela il nous faut rappeler la théorie des $\phi$-espaces de Drinfeld.

\subsection{Rappels sur les $\phi$-espaces}

La théorie des $\phi$-espaces est due à Drinfeld. Une référence utile est l'appendice de [17].

DÉFINITION 1. - Un $\phi$-espace est un $F \otimes_{\mathbb{F}_{q}} k$-espace vectoriel $V$ muni d'une application $\operatorname{id}_{F} \otimes \sigma$-linéaire bijective $\phi: V \rightarrow V$. Un morphisme de $\phi$-espaces $\alpha:\left(V_{1}, \phi_{1}\right) \rightarrow\left(V_{2}, \phi_{2}\right)$ est une application $F \otimes_{\mathbb{F}_{q}} k$-linéaire $\alpha: V_{1} \rightarrow V_{2}$ telle que $\phi_{2} \circ \alpha=\alpha \circ \phi_{1}$. On note $\mathcal{F}$ la catégorie des $\phi$-espaces.

Drinfeld a attaché à tous $\phi$-espaces un invariant qu'il appelle $\phi$-paire dont on va rappeler la définition.

Soit $E$ une extension de $F$. Notons $X_{E}$ le normalisé de la courbe $X$ dans $E$. Notons $\operatorname{Div}^{0}\left(X_{E}\right)$ le groupe des diviseurs de degré 0 sur la courbe $X_{E}$. On a l'homomorphisme de groupes abéliens

$$
E^{\times} \rightarrow \operatorname{Div}^{0}\left(X_{E}\right)
$$

qui associe à une fonction rationnelle non nulle $f \in E$ sur $X_{E}$ son $\operatorname{diviseur} \operatorname{div}(f)$. Le conoyau de cette application, le groupe des diviseurs de $X_{E}$ de degré 0 modulo les diviseurs principaux, est le groupe des $\mathbb{F}_{q}$-points de la jacobienne de $X_{E}$. En particulier, c'est un groupe abélien fini. Son noyau, étant le groupe des fonctions constantes non nulles en $X_{E}$, est aussi un groupe abélien fini. Il s'ensuit que l'homomorphisme de $\mathbb{Q}$-espaces vectoriels

$$
E^{\times} \otimes \mathbb{Q} \rightarrow \operatorname{Div}^{0}\left(X_{E}\right) \otimes \mathbb{Q}
$$

$4^{\mathrm{e}}$ SÉRIE - TOME $39-2006-\mathrm{N}^{\circ} 2$ 
qui s'en déduit est un isomorphisme.

DÉfinition 2. - Une $\phi$-paire est une paire $(E, Q)$ où $E$ est une $F$-algèbre finie et où $Q$ est un élément de $E^{\times} \otimes \mathbb{Q}$.

Soit $\mathcal{E}^{\prime}$ la catégorie dont les objets sont des $\phi$-paires $(E, Q)$, une flèche $(E, Q) \rightarrow\left(E^{\prime}, Q^{\prime}\right)$ dans $\mathcal{E}^{\prime}$ est un homomorphisme injectif de $F$-algèbres $\phi: E \rightarrow E^{\prime}$ tel que $\phi(Q)=Q^{\prime}$. On appelle la catégorie des $\phi$-paires, la catégorie $\mathcal{E}$ obtenue de $\mathcal{E}^{\prime}$ en inversant toutes les flèches de $\mathcal{E}^{\prime}$.

Soit $(E, Q)$ un objet de $\mathcal{E}$. Parmi les $F$-sous-algèbres $E^{\prime}$ de $E$ telles que $Q$ soit dans l'image de l'inclusion évidente $E^{\prime \times} \otimes \mathbb{Q} \hookrightarrow E^{\times} \otimes \mathbb{Q}$, il en existe une qui est contenue dans toutes les autres; notons-la $\widetilde{E}$. Si $E=\widetilde{E}$, la $\phi$-paire $(E, \phi)$ sera dite minimale. Si $(\widetilde{E}, Q)$ est une $\phi$-paire minimale, on vérifie facilement que $\widetilde{E}$ est une $F$-algèbre étale. Dans $\mathcal{E}$, toute $\phi$-paire $(E, Q)$ est isomorphe à une $\phi$-paire $(\widetilde{E}, Q)$ minimale, unique à isomorphismes non uniques près.

Soit $(E, Q)$ une $\phi$-paire minimale où $E$ est un corps. Choisissons un plongement de $E$ dans une clôture séparable $F^{s e p}$ de $F$. L'image de $Q$ dans $\left(F^{s e p}\right)^{\times} \otimes \mathbb{Q}$ est bien déterminée à $\mathrm{Gal}\left(F^{\text {sep }} / F\right)$-conjugaison près. Il est clair qu'on peut retrouver la classe d'isomorphisme de $(E, Q)$ à partir de cet élément de $\left[\left(F^{s e p}\right)^{\times} \otimes \mathbb{Q}\right] / \operatorname{Gal}\left(F^{s e p} / F\right)$.

Proposition 3. - L'ensemble des classes d'isomorphisme des $\phi$-paires $(E, Q)$ où E est un corps est en bijection naturelle avec $\left[\left(F^{s e p}\right)^{\times} \otimes \mathbb{Q}\right] / \mathrm{Gal}\left(F^{s e p} / F\right)$.

On peut construire un foncteur de la catégorie $\mathcal{F}$ des $\phi$-espaces dans la catégorie $\mathcal{E}$ des $\phi$-paires comme suit. Soit $(V, \phi)$ un $\phi$-espace. Considérons l'ensemble des paires $\left(n, V_{n}\right)$ formée

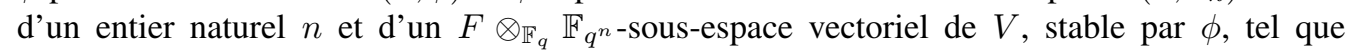
$V=V_{n} \otimes_{\mathbb{F}_{q^{n}}} k$. Cet ensemble possède un ordre partiel : $\left(n, V_{n}\right) \leqslant\left(n^{\prime}, V_{n^{\prime}}\right)$ si et seulement si $n$ divise $n^{\prime}$ et $V_{n^{\prime}}=V_{n} \otimes_{\mathbb{F}_{q^{n}}} \mathbb{F}_{q^{n^{\prime}}}$. Cet ordre est filtrant.

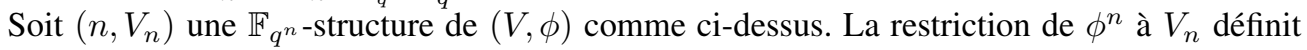
alors un automorphisme linéaire de $V_{n}$. Notons $E$ la $F$-sous-algèbre de $\operatorname{End}\left(V_{n}\right)$ engendrée par la restriction de $\phi^{n}$ à $V_{n}$. Notons $Q$ l'élément de $\operatorname{Div}^{0}\left(X_{E}\right) \otimes \mathbb{Q}$ telle que

$$
n Q=\operatorname{div}\left(\left.\phi^{n}\right|_{V_{n}}\right)
$$

On obtient une $\phi$-paire $(E, Q)$.

Si $\left(n, V_{n}\right) \leqslant\left(n^{\prime}, V_{n}^{\prime}\right)$ et $\left(E^{\prime}, Q^{\prime}\right)$ est la $\phi$-paire associée à $\left(n^{\prime}, V_{n}^{\prime}\right)$, on a visiblement un $F$-homomorphisme canonique $E^{\prime} \rightarrow E$ qui envoie $Q^{\prime}$ sur $Q$. Ceci définit un isomorphisme canonique dans la catégorie $\mathcal{E}$. Puisque l'ordre $\left(n, V_{n}\right) \leqslant\left(n^{\prime}, V_{n}^{\prime}\right)$ est filtrant, les différentes $\phi$-paires $(E, Q)$ qu'on a construit se diffèrent par un unique isomorphisme de $\mathcal{E}$. On obtient ainsi un foncteur $\mathcal{F} \rightarrow \mathcal{E}$.

Rappelons un théorème de Drinfeld.

THÉORÈME 4. -

(1) La catégorie des $\phi$-espaces sur $k$ est abélienne F-linéaire et semi-simple.

(2) Le foncteur $\mathcal{F} \rightarrow \mathcal{E}$ ci-dessus induit une bijection entre l'ensemble des classes d'isomorphisme de $\phi$-espaces irréductibles et l'ensemble des classes d'isomorphisme dans $\mathcal{E}$ des $\phi$-paires $(E, Q)$ où E est un corps.

(3) Pour une extension finie $E$ de $F$ et un élément $Q \in \operatorname{Div}^{0}\left(X_{E}\right) \otimes \mathbb{Q}$, supposons que la $\phi$-paire $(E, Q)$ est minimale. Notons b le plus petit entier naturel tel que

$$
Q \in \frac{1}{b} \operatorname{Div}^{0}\left(X_{E}\right) .
$$


Soit $(V, \phi)$ le $\phi$-espace irréductible qui correspond à la $\phi$-paire $(E, Q)$; on a

$$
\operatorname{dim}_{F \otimes_{\mathbb{F}_{q}} k}(V)=[E: F] b .
$$

L'algèbre $\operatorname{End}(V, \phi)$ est une algèbre à division centrale sur $E$ de dimension $b^{2}$ et d'invariant

$$
\begin{array}{r}
\operatorname{inv}(\operatorname{End}(V, \phi)) \in \operatorname{Div}^{0}\left(X_{E}\right) \otimes \mathbb{Q} / \mathbb{Z} \\
\text { égal à l'image de }-Q \in \operatorname{Div}^{0}\left(X_{E}\right) \otimes \mathbb{Q} \text { dans } \operatorname{Div}^{0}\left(X_{E}\right) \otimes \mathbb{Q} / \mathbb{Z} .
\end{array}
$$

La classe d'isomorphisme d'un $\phi$-espace irréductible $(V, \phi)$ est donc déterminée par une $\phi$-paire minimale $(E, Q)$, et donc en définitive, est déterminée par l'image de $Q$ dans $\left[\left(F^{s e p}\right)^{\times} \otimes\right.$ $\mathbb{Q}] / \operatorname{Gal}\left(F^{s e p} / F\right)$.

\subsection{La classe de conjugaison $\gamma_{0}$}

Soit $\left(V ; \tau, \tau^{\prime}\right)$ un objet de la catégorie $C\left(T, T^{\prime} ; s\right)$. On peut produire une bijection $D \otimes_{\mathbb{F}_{q}} k$ linéaire de $V$ à partir des applications semi-linéaires $\tau$ et $\tau^{\prime}$ en prenant le composé

$$
\gamma=\tau^{s} \tau^{\prime-1}: V \rightarrow V
$$

En choisissant une rigidification $V \stackrel{\sim}{\longrightarrow} D^{o p} \otimes_{\mathbb{F}_{q}} k, \gamma$ définit un élément de

$$
G\left(F \otimes_{\mathbb{F}_{q}} k\right)=\left(D \otimes_{\mathbb{F}_{q}} k\right)^{\times} .
$$

Nous notons $\gamma_{0}$ la classe de conjugaison de $\gamma$ dans $G\left(F \otimes_{\mathbb{F}_{q}} k\right)$ qui, bien entendu, ne dépend pas de la rigidification choisie.

LEMme 1. - La classe de conjugaison $\gamma_{0}$ de $G\left(F \otimes_{\mathbb{F}_{q}} k\right)$ est $\sigma$-invariante.

Démonstration. - On déduit de l'hypothèse de commutation $\tau \tau^{\prime}=\tau^{\prime} \tau$ un carré commutatif

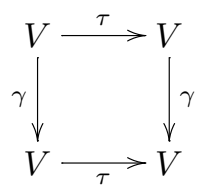

Que $\gamma$ commute avec un isomorphisme $\sigma$-linéaire implique que $\gamma$ et $\sigma(\gamma)$ sont conjugués.

Proposition 2. - La classe de conjugaison $\gamma_{0}$ dans $G\left(F \otimes_{\mathbb{F}_{q}} k\right)$ rencontre $G(F)$. De plus, cette intersection est formée d'une seule classe de $G(F)$-conjugaison.

Démonstration. - $G$ est une forme intérieure de $\mathrm{GL}(d)$ de sorte qu'il existe une injection canonique Galois équivariante de l'ensemble des classes de conjugaison de $G\left(F \otimes_{\mathbb{F}_{q}} k\right)$ dans l'ensemble des classes de conjugaison $\mathrm{GL}_{d}\left(F \otimes_{\mathbb{F}_{q}} k\right)$. On peut donc transférer $\gamma_{0}$ en une classe de conjugaison de $\mathrm{GL}_{d}\left(F \otimes_{\mathbb{F}_{q}} k\right)$ qui est $\sigma$-invariante. Celle-ci provient donc d'une unique classe de conjugaison de $\mathrm{GL}_{d}(F)$.

Le problème consiste donc à vérifier que cette classe de conjugaison $\tilde{\gamma}$ de $\mathrm{GL}_{d}(F)$ provient de $G(F)$. Il revient au même de trouver un plongement de l'algèbre $F[\tilde{\gamma}]$ engendrée par $\tilde{\gamma}$ dans $D^{o p}$. D'après [14, lemme 4, III.3], ceci est un problème local en des places $v$ de $X$ où $D$ se ramifie, c'est-à-dire $\gamma_{0}$ vue comme classe de conjugaison de $\mathrm{GL}_{d}\left(F_{v}\right)$ provient de $G\left(F_{v}\right)$. Or,

$4^{\text {e }}$ SÉRIE - TOME $39-2006-\mathrm{N}^{\circ} 2$ 
$T$ évite ces places par hypothèse si bien que la classe de conjugaison $\gamma_{0}$ vue dans $G\left(F_{v} \otimes_{\mathbb{F}_{q}} k\right)$ vient d'une classe de conjugaison dans $G\left(F_{v}\right)$.

Choisissons un représentant de la classe de conjugaison $\gamma_{0}$ dans $G(F)=D^{\times}$qu'on va encore noter $\gamma_{0}$. Notons $E=F\left[\gamma_{0}\right]$ la sous- $F$-algèbre de $D$ engendrée par $\gamma_{0}$. Puisque $D$ est une algèbre à division centrale sur $F, E$ est un corps. Notons $X_{E}$ le normalisé de $X$ dans $E$. Notons $\pi: X_{E} \rightarrow X$.

DÉFINITION 3. - La classe de conjugaison $\gamma_{0}$ de $D^{\times}$est dite $\left(T, T^{\prime}\right)$-admissible si le diviseur $\operatorname{div}\left(\gamma_{0}\right)$ de $X_{E}$ est supporté par $\pi^{-1}\left(T \cup T^{\prime}\right)$ et si en décomposant $\operatorname{div}\left(\gamma_{0}\right)$ en somme

$$
\operatorname{div}\left(\gamma_{0}\right)=\operatorname{div}_{T}\left(\gamma_{0}\right)+\operatorname{div}_{T^{\prime}}\left(\gamma_{0}\right)
$$

d'un diviseur supporté par $\pi^{-1}(T)$ et d'un diviseur supporté par $\pi^{-1}\left(T^{\prime}\right)$, les diviseurs $\operatorname{div}_{T}\left(\gamma_{0}\right)$ et $\operatorname{div}_{T^{\prime}}\left(\gamma_{0}\right)$ sont tous les deux de degré 0 .

Proposition 4. - Soit $\left(V ; \tau, \tau^{\prime}\right)$ un objet de $C\left(T, T^{\prime} ; s\right)$. Soit $\gamma=\tau^{s} \tau^{-1}$. Soit $\gamma_{0}$ la classe de conjugaison de $G(F)$ qui est $G\left(F \otimes_{\mathbb{F}_{q}} \overline{\mathbb{F}}_{q}\right)$-conjuguée à $\gamma$. Alors $\gamma_{0}$ est une classe de conjugaison de $G(F)$ qui est $\left(T, T^{\prime}\right)$-admissible.

On note encore $\gamma_{0} \in D^{\times}$un représentant de cette classe de conjugaison $\gamma_{0}$; soit $E_{0}$ la F-sousalgèbre de $D$ engendrée par $\gamma_{0}$. Soit

$$
\operatorname{div}\left(\gamma_{0}\right)=\operatorname{div}_{T}\left(\gamma_{0}\right)+\operatorname{div}_{T^{\prime}}\left(\gamma_{0}\right)
$$

la décomposition du diviseur sur $X_{E_{0}}$ associé à la fonction rationnelle $\gamma_{0}$, en la somme d'un diviseur supporté par $T$ avec un diviseur supporté par $T^{\prime}$. Alors, dans la catégorie $\mathcal{E}$, la $\phi$-paire

$$
\left(E, \frac{\operatorname{div}_{T}\left(\gamma_{0}\right)}{s}\right)
$$

est isomorphe à la $\phi$-paire associée au $\phi$-espace $(V, \tau)$.

Démonstration. - Il existe un entier naturel $n$ assez divisible, en particulier $s \mid n$, tel qu'il existe

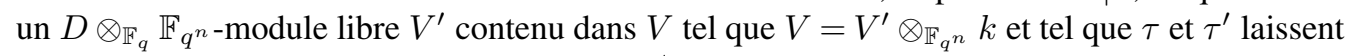
stable $V^{\prime}$. Il est clair que $\left(\left.\tau\right|_{V^{\prime}}\right)^{n}$ et $\left(\left.\tau^{\prime}\right|_{V^{\prime}}\right)^{n / s}$ sont des automorphismes $D \otimes_{\mathbb{F}_{q}} \mathbb{F}_{q^{n}}$-linéaires de $V^{\prime}$. On a

$$
\left(\left.\gamma\right|_{V^{\prime}}\right)^{n / s}=\left(\left.\tau\right|_{V^{\prime}}\right)^{n}\left(\left.\tau^{\prime}\right|_{V^{\prime}}\right)^{-n / s} .
$$

Soit $E$ la $F$-sous-algèbre de $\operatorname{End}\left(V^{\prime}\right)$ engendrée par $\left(\left.\tau\right|_{V^{\prime}}\right)^{n}$ et $\left(\left.\tau^{\prime}\right|_{V^{\prime}}\right)^{n}$; c'est un corps puisque $\left(V ; \tau, \tau^{\prime}\right)$ est irréductible d'après la proposition 2 de 4.2 .

Soit $X_{E}$ le normalisé de $X$ dans $E$. On a donc une égalité de diviseurs sur $X_{E}$

$$
\operatorname{div}\left(\left(\left.\gamma\right|_{V^{\prime}}\right)^{n / s}\right)=\operatorname{div}\left(\left(\left.\tau\right|_{V^{\prime}}\right)^{n}\right)+\operatorname{div}\left(\left(\left.\tau^{\prime}\right|_{V^{\prime}}\right)^{-n / s}\right) .
$$

On va utiliser le lemme suivant.

LEMme 5. - Dans l'expression ci-dessus, $\operatorname{div}\left(\left(\left.\tau\right|_{V^{\prime}}\right)^{n}\right)$ est supporté par $T$ et $\operatorname{div}\left(\left(\left.\tau^{\prime}\right|_{V^{\prime}}\right)^{-n / s}\right)$ est supporté par $T^{\prime}$.

Démonstration du lemme 5. - Soit $x \in|X-T|$. Par hypothèse $\left(V_{x}, \tau_{x}\right)$ est isomorphe à $\left(D_{x} \otimes_{\mathbb{F}_{q}} k, \operatorname{id}_{D_{x}} \otimes \sigma\right)$. Il existe donc un entier $n^{\prime \prime}$ divisible par $n$ tel que $\left(V_{x}^{\prime}, \tau_{x}\right) \otimes_{\mathbb{F}_{q^{n}}} \mathbb{F}_{q^{n^{\prime \prime}}}$ 
est isomorphe à $\left(D_{x} \otimes_{\mathbb{F}_{q}} \mathbb{F}_{q^{n^{\prime \prime}}}, \operatorname{id}_{D_{x}} \otimes \sigma\right)$. L'automorphisme linéaire de $\left(V_{x}^{\prime}, \tau_{x}\right) \otimes_{\mathbb{F}_{q^{n}}} \mathbb{F}_{q^{n^{\prime \prime}}}$

$$
\left(\left.\tau_{x}\right|_{V_{x}^{\prime} \otimes_{\mathbb{F}_{q} n^{n}} \mathbb{F}_{q^{n^{\prime \prime}}}}\right)^{n^{\prime \prime}}=\left(\left(\left.\tau_{x}\right|_{V_{x}^{\prime} \otimes_{\mathbb{F}_{q}{ }^{n}} \mathbb{F}_{q^{n^{\prime \prime}}}}\right)^{n^{\prime}}\right)^{n^{\prime \prime} / n^{\prime}}
$$

est donc égal à l'identité. Il s'ensuit que $x$ n'appartient pas au support de $\operatorname{div}\left(\left(\left.\tau\right|_{V^{\prime}}\right)^{n}\right)$. Le même argument vaut pour $\tau^{\prime}$ et pour $x \in\left|X-T^{\prime}\right|$.

Suite de la démonstration de la proposition 4. - Il est clair, grâce au lemme ci-dessus, que $\operatorname{div}\left(\left(\left.\gamma\right|_{V^{\prime}}\right)^{n}\right)$ est un diviseur supporté par $T \cup T^{\prime}$, dont la partie supportée par $T$ est $\operatorname{div}\left(\left(\left.\tau\right|_{V^{\prime}}\right)^{n s}\right)$ et dont la partie supportée par $T^{\prime} \operatorname{est} \operatorname{div}\left(\left(\left.\tau^{\prime}\right|_{V^{\prime}}\right)^{-n}\right)$. Puisque ce sont, tous les deux, des diviseurs principaux, leurs degrés sont nuls. On en déduit que la classe de conjugaison $\gamma_{0}$ est $\left(T, T^{\prime}\right)$ admissible car, dans la définition de la propriété $\left(T, T^{\prime}\right)$-admissible, il est loisible de remplacer l'extension engendrée par $\gamma_{0}$ par n'importe quelle extension qui la contient, et de remplacer $\gamma_{0}$ par une puissance de $\gamma_{0}$.

Il reste à démontrer que la $\phi$-paire $\left(E_{0}, \operatorname{div}_{T}\left(\gamma_{0}\right) / s\right)$ est isomorphe à la $\phi$-paire associée au $\phi$-espace $(V, \tau)$, dans la catégorie $\mathcal{E}$. Il existe un homomorphisme d'algèbres $E_{0} \rightarrow E$ qui envoie $\gamma_{0}$ sur $\gamma$ de sorte qu'on se ramène à démontrer l'assertion pour la $\phi$-paire $\left(E, \operatorname{div}_{T}(\gamma) / s\right)$.

Soit $E^{\prime}$ la $F$-sous-algèbre de $\operatorname{End}\left(V^{\prime}\right)$ engendrée par $\left(\left.\tau\right|_{V^{\prime}}\right)^{n}$. Par construction la $\phi$-paire associée au $\phi$-espace $(V ; \tau)$ est $\left(E^{\prime}, \operatorname{div}\left(\left(\left.\tau\right|_{V^{\prime}}\right)^{n}\right) / n\right)$. Il est clair que $E^{\prime}$ est une sous-extension de $E$ qui est la $F$-sous-algèbre de $\operatorname{End}\left(V^{\prime}\right)$ engendrée $\operatorname{par}\left(\tau \mid{ }_{V^{\prime}}\right)^{n}$ et $\left(\left.\tau^{\prime}\right|_{V^{\prime}}\right)^{n}$ de sorte que dans la catégorie $\mathcal{E}$, les $\phi$-paires $\left(E^{\prime}, \operatorname{div}\left(\left(\left.\tau\right|_{V^{\prime}}\right)^{n}\right) / n\right)$ et $\left(E, \operatorname{div}\left(\left(\left.\tau\right|_{V^{\prime}}\right)^{n}\right) / n\right)$ sont isomorphes. Sur $X_{E}$, l'égalité

$$
\frac{\operatorname{div}\left(\left(\left.\tau\right|_{V^{\prime}}\right)^{n}\right)}{n}=\frac{\operatorname{div}_{T}(\gamma)}{s}
$$

se déduit du lemme 5 .

Soit $x \in|T|$. On a une application $s$-norme de l'ensemble des classes de $\sigma$-conjugaison dans $G\left(F_{x} \otimes_{\mathbb{F}_{q}} \mathbb{F}_{q^{s}}\right)$ dans l'ensemble des classes de conjugaison stable de $G\left(F_{x}\right)$. En une place $x \in|T|$ donc $x \in\left|X^{\prime}\right|, G$ est isomorphe à $\mathrm{GL}_{d}$ de sorte que les classes de conjugaison stable sont simplement des classes de conjugaison. On a donc une application $s$-norme

$$
\mathrm{N}_{x, s}: G\left(F_{x} \otimes_{\mathbb{F}_{q}} \mathbb{F}_{q^{s}}\right) / \sigma \text {-conj. } \rightarrow G\left(F_{x}\right) / \text { conj. }
$$

DÉfinition 6. - Soit $s \in \mathbb{N}$. La classe de conjugaison $\gamma_{0}$ de $G(F)$ est dite une $s$-norme en $T$ si pour tout $x \in|T|, \gamma_{0}$ vue comme classe de conjugaison de $G\left(F_{x}\right)$ est dans l'image de $\mathrm{N}_{x, s}$.

Proposition 7. - Soit $\left(V ; \tau, \tau^{\prime}\right)$ un objet de $C\left(T, T^{\prime} ; s\right)$. Soit $\gamma=\tau^{s} \tau^{\prime-1}$. Soit $\gamma_{0}$ la classe de conjugaison de $G(F)$ qui est $G\left(F \otimes_{\mathbb{F}_{q}} k\right)$-conjuguée à $\gamma$. Alors $\gamma_{0}$ est une s-norme en $T$.

Démonstration. - Soit $x \in|T|$. Par hypothèse $x \notin\left|T^{\prime}\right|$. Le complété $V_{x}$ de $V$ en $x$ est un $D_{x} \otimes_{\mathbb{F}_{q}} k$-module libre de rang 1 muni d'une bijection $\sigma$-linéaire $\tau_{x}$ et d'une bijection $\sigma^{s}$-linéaire $\tau_{x}^{\prime}$. Puisque $x \notin T^{\prime}$, la paire $\left(V_{x}, \tau_{x}^{\prime}\right)$ est isomorphe à $\left(\left(D_{x} \widehat{\otimes}_{\mathbb{F}_{q}} \mathbb{F}_{q^{s}}\right) \widehat{\otimes}_{\mathbb{F}_{q^{s}}} k, \mathrm{id}_{D_{x} \widehat{\otimes}_{\mathbb{F}_{q}} \mathbb{F}_{q^{s}}} \widehat{\otimes} \sigma^{s}\right)$. En prenant les vecteurs fixes sous $\tau_{x}^{\prime}$, on obtient donc un $D_{x} \widehat{\otimes}_{\mathbb{F}_{q}} \mathbb{F}_{q^{s}}$-module libre $V_{x}^{\prime}$. Puisque $\tau_{x}$ commute à $\tau_{x}^{\prime}, \tau_{x}$ induit sur $V_{x}^{\prime}$ une application $\operatorname{id}_{D_{x}} \widehat{\otimes} \sigma$-linéaire qui définit une classe de $\sigma$-conjugaison dans $G\left(F_{x} \widehat{\otimes}_{\mathbb{F}_{q}} \mathbb{F}_{q^{s}}\right)$ qu'on notera $\delta_{x}$. Par construction $\left.\tau_{x}^{\prime}\right|_{V_{x}^{\prime}}=1$ de sorte que $\left.\gamma_{x}\right|_{V_{x}^{\prime}}=\left.\tau_{x}^{s}\right|_{V_{x}^{\prime}}$. On en déduit que $\gamma_{0}$ vue comme classe de conjugaison de $G\left(F_{x}\right)$ est la $s$-norme de la classe de $\sigma$-conjugaison $\delta_{x}$.

$4^{\text {e }}$ SÉRIE - TOME $39-2006-\mathrm{N}^{\circ} 2$ 


\subsection{Les classes d'isogénie}

On a vu qu'un objet $\left(V ; \tau, \tau^{\prime}\right)$ de $C\left(T, T^{\prime} ; s\right)$ détermine une classe de conjugaison $\gamma_{0}$ dans $G(F)$ qui est $\left(T, T^{\prime}\right)$-admissible et $s$-norme en $T$. On va voir que, réciproquement, une classe de conjugaison de $G(F)$ qui est $\left(T, T^{\prime}\right)$-admissible et $s$-norme en $T$, détermine uniquement une classe d'isomorphisme de $C\left(T, T^{\prime} ; s\right)$. Ceci constituera l'analogue de la théorie de Honda-Tate dans notre problème de comptage. Il s'agit encore d'une variante d'un théorème de Drinfeld, déjà généralisé par Laumon, Rapoport, Stuhler et par Lafforgue à d'autres contextes.

THÉORÈME 1. - L'application qui associe à un objet $\left(V ; \tau, \tau^{\prime}\right)$ de $C\left(T, T^{\prime} ; s\right)$ la classe de conjugaison $\gamma_{0}$ de $G(F)$ conjuguée dans $G\left(F \otimes_{\mathbb{F}_{q}} k\right) \grave{a} \gamma=\tau^{s} \tau^{\prime-1}$, définit une bijection de l'ensemble des classes d'isomorphisme de $C\left(T, T^{\prime} ; s\right)$ sur l'ensemble des classes de conjugaison de $G(F)$ qui sont $\left(T, T^{\prime}\right)$-admissibles et s-normes en $T$.

Démonstration. - Il s'agit de vérifier que l'application $\left(V ; \tau, \tau^{\prime}\right) \mapsto \gamma_{0}$ est injective et surjective. Vérifions d'abord la surjectivité.

Soit $\gamma_{0}$ une classe de conjugaison de $G(F)$ qui est $\left(T, T^{\prime}\right)$-admissible et $s$-norme en $T$. On va construire un objet $\left(V ; \tau, \tau^{\prime}\right)$ de $C\left(T, T^{\prime} ; s\right)$ dont la classe de conjugaison dans $G(F)$ associée est $\gamma_{0}$ en plusieurs étapes.

Étape 1 : le $\phi$-espace irréductible $(W, \psi)$ facteur de $(V, \tau)$. Prenons un représentant de la classe de conjugaison $\gamma_{0}$ qu'on note $\gamma_{0} \in D^{\times}$. Soit $E=F\left[\gamma_{0}\right]$ la sous- $F$-algèbre de $D$ engendrée par $\gamma_{0}$. C'est une extension de corps de $F$ puisque $D$ est une algèbre à division. Le degré de cette extension $e=[E: F]$ divise le rang de $D$ qui est égal à $d$.

Notons $X_{E}$ le normalisé de $X$ dans $E$. D'après l'hypothèse de $\left(T, T^{\prime}\right)$-admissibilité, le diviseur $\operatorname{div}\left(\gamma_{0}\right)$ se casse en somme de deux diviseurs de degré 0 supportés respectivement par $T$ et $T^{\prime}$

$$
\operatorname{div}\left(\gamma_{0}\right)=\operatorname{div}_{T}\left(\gamma_{0}\right)+\operatorname{div}_{T^{\prime}}\left(\gamma_{0}\right) .
$$

Soit $E_{T}$ le plus petit sous-corps de $E$ tel que $\operatorname{Div}^{0}\left(X_{E_{T}}\right) \otimes \mathbb{Q}$ contient encore la $\mathbb{Q}$-droite engendrée par $\operatorname{div}_{T}\left(\gamma_{0}\right)$. Notons $e_{T}=\left[E_{T}: F\right]$.

La paire $\left(E_{T}, \operatorname{div}_{T}\left(\gamma_{0}\right) / s\right)$ est alors une $\phi$-paire minimale. Soit $(W, \psi)$ un $\phi$-espace irréductible correspondant à $\left(E_{T}, \operatorname{div}_{T}\left(\gamma_{0}\right) / s\right)$; il est bien défini à isomorphisme près. Soit $b$ le plus petit entier naturel tel que

$$
\frac{\operatorname{div}_{T}\left(\gamma_{0}\right)}{s} \in \frac{1}{b} \operatorname{Div}^{0}\left(X_{E_{T}}\right)
$$

D'après le théorème 4 de 4.3 , End $(W, \psi)$ est une algèbre à division centrale sur $E_{T}$ de dimension $b^{2}$ sur $E_{T}$ et

$$
\operatorname{dim}_{F \otimes k}(W)=b e_{T}
$$

Étape 2 : structure de $D^{o p}$-module sur le bon multiple de $(W, \psi)$. Considérons le multiple $(V, \tau)=(W, \psi)^{r}$ avec $r=d^{2} / b e_{T}$. L'espace vectoriel $V$ a donc la bonne dimension $d^{2}$ sur $F \otimes k$. Notons que

$$
\operatorname{dim}_{E_{T}} M_{r}(\operatorname{End}(W, \psi))=r^{2} b^{2}=\frac{d^{4}}{e_{T}^{2}} .
$$


Pour donner à $V$ une structure de $D^{o p}$-module par rapport à laquelle $\tau$ est $\operatorname{id}_{D} \otimes \sigma$-linéaire, il suffit de construire un plongement

$$
D^{o p} \hookrightarrow M_{r}(\operatorname{End}(W, \psi))
$$

ou, ce qui est équivalent, un plongement d'algèbres simples centrales sur $E_{T}$

$$
D^{o p} \otimes_{F} E_{T} \hookrightarrow M_{r}(\operatorname{End}(W, \psi)) .
$$

Ceci est encore équivalent à l'existence d'une autre algèbre simple centrale $C$ sur $E_{T}$ telle que

$$
\left(D^{o p} \otimes_{F} E_{T}\right) \otimes C \simeq M_{r}(\operatorname{End}(W, \psi)) .
$$

Une telle algèbre simple centrale $E$ doit avoir la dimension

$$
\operatorname{dim}_{E_{T}}(C)=\frac{\operatorname{dim}_{E_{T}} M_{r}(\operatorname{End}(W, \psi))}{\operatorname{dim}_{E_{T}}\left(D^{o p} \otimes_{F} E_{T}\right)}=\frac{d^{2}}{e_{T}^{2}}
$$

et l'invariant

$$
\operatorname{inv}(C)=\operatorname{inv}(\operatorname{End}(W, \psi))-\operatorname{inv}\left(D^{o p} \otimes_{F} E_{T}\right)
$$

l'égalité étant prise dans $\operatorname{Div}^{0}\left(X_{E_{T}}\right) \otimes \mathbb{Q} / \mathbb{Z}$. Pour que $C$ existe il faut et il suffit que

$$
\operatorname{inv}(\operatorname{End}(W, \psi))-\operatorname{inv}\left(D^{o p} \otimes_{F} E_{T}\right) \in \frac{e_{T}}{d} \operatorname{Div}^{0}\left(X_{E_{T}}\right) / \operatorname{Div}^{0}\left(X_{E_{T}}\right) .
$$

Rappelons un lemme [14, III.4, lemme 4].

Lemme 2.-Soient $E$ une extension de $F$ et $A$ une algèbre simple centrale sur $E$ de dimension $d^{2}$. Soit $E^{\prime}$ une extension de $E$ de degré $e^{\prime}$. Pour qu'il existe un plongement de $E^{\prime} \hookrightarrow A$, il faut et il suffit que $e^{\prime}$ divise d et que l'image de $\operatorname{inv}(A)$ dans $\operatorname{Div}^{0}\left(X_{E^{\prime}}\right) \otimes \mathbb{Q} / \mathbb{Z}$ soit dans

$$
\operatorname{inv}\left(A \otimes_{E} E^{\prime}\right) \in \frac{e^{\prime}}{d} \operatorname{Div}^{0}\left(X_{E^{\prime}}\right) / \operatorname{Div}^{0}\left(X_{E^{\prime}}\right)
$$

On a les inclusions $E_{T} \subset E \subset D^{o p}$ de sorte qu'en vertu du lemme ci-dessus, on a

$$
\operatorname{inv}\left(D^{o p} \otimes_{F} E_{T}\right) \in \frac{e_{T}}{d} \operatorname{Div}^{0}\left(X_{E_{T}}\right) / \operatorname{Div}^{0}\left(X_{E_{T}}\right) .
$$

Par ailleurs, grâce au théorème 4 de 4.3, on sait que l'invariant

$$
\operatorname{inv}(\operatorname{End}(W, \psi)) \in \operatorname{Div}^{0}\left(X_{E_{T}}\right) \otimes \mathbb{Q} / \mathbb{Z}
$$

est égal à l'image de $-\operatorname{div}_{T}\left(\gamma_{0}\right) / s \in \operatorname{Div}^{0}\left(X_{E_{T}}\right) \otimes \mathbb{Q}$. Il reste donc à démontrer que

$$
\frac{\operatorname{div}_{T}\left(\gamma_{0}\right)}{s} \in \frac{e_{T}}{d} \operatorname{Div}^{0}\left(X_{E_{T}}\right) .
$$

On va utiliser le lemme suivant.

$4^{\text {e }}$ SÉRIE - TOME $39-2006-\mathrm{N}^{\circ} 2$ 
LEMme 3. - Soit $\gamma_{0}$ un élément de $D^{\times}$dont la classe de conjugaison est une s-norme en $T$. Soit E la F-sous-algèbre de $D$ engendrée par $\gamma_{0}$. Notons $e=[E: F]$. Alors on a

$$
\operatorname{div}_{T}\left(\gamma_{0}\right) \in \frac{e}{d} \operatorname{Div}^{0}\left(X_{E}\right)
$$

où $\operatorname{div}_{T}\left(\gamma_{0}\right)$ est la partie $\operatorname{div}\left(\gamma_{0}\right)$ supportée par $T$.

Démonstration. - Soit $D^{\prime}$ le centralisateur de $E$ dans $D$. C'est une algèbre à division centrale de rang $d / e$ sur $E$. En remplaçant $D$ par $D^{\prime}$ et $F$ par $E$, on peut supposer que l'élément $\gamma_{0}$ appartient à $F$. En une place $x \in|T|, D_{x} \simeq \mathrm{GL}_{d}\left(F_{x}\right)$. Il existe donc $\delta \in \mathrm{GL}_{d}\left(F_{x} \otimes_{\mathbb{F}_{q}} \mathbb{F}_{q^{s}}\right)$ tel que

$$
\gamma_{0}=\delta \sigma(\delta) \cdots \sigma^{s-1}(\delta)
$$

On en déduit que

$$
\operatorname{det}\left(\gamma_{0}\right)=\mathrm{N}_{s}(\operatorname{det}(\delta))
$$

de sorte que la valuation de $\operatorname{det}\left(\gamma_{0}\right)$ est divisible par $s$. Or vu comme élément du centre $F_{x}$, on a $\operatorname{det}\left(\gamma_{0}\right)=\gamma_{0}^{d}$ d'où le lemme.

Suite de l'étape 2. L'inclusion $E_{T} \subset E$ induit un revêtement $\pi: X_{E} \rightarrow X_{E_{T}}$ de degré $e / e_{T}$. L'application injective $\pi^{*}: \operatorname{Div}^{0}\left(X_{E_{T}}\right) \rightarrow \operatorname{Div}^{0}\left(X_{E}\right)$ induit par tensorisation avec $\mathbb{Q}$ l'application usuelle $E_{T}^{\times} \otimes \mathbb{Q} \rightarrow E^{\times} \otimes \mathbb{Q}$. Mais on a aussi l'application $\pi_{*}: \operatorname{Div}^{0}\left(X_{E}\right) \rightarrow$ $\operatorname{Div}^{0}\left(X_{E_{T}}\right)$. Pour tout $Q \in \operatorname{Div}^{0}\left(X_{E_{T}}\right)$, on a $\pi_{*}\left(\pi^{*}(Q)\right)=\frac{e}{e_{T}} Q$. On en déduit que si

$$
Q \in \operatorname{Div}^{0}\left(X_{E}\right) \cap \operatorname{Im}\left[\operatorname{Div}^{0}\left(X_{E_{T}}\right) \otimes \mathbb{Q}\right]
$$

alors $\pi^{*}\left(\pi_{*}(Q)\right)$ est aussi égale à $\frac{e}{e_{T}} Q$.

D'après le lemme, il existe un diviseur $Q \in \operatorname{Div}^{0}\left(X_{E}\right)$ tel que $\operatorname{div}_{T}\left(\gamma_{0}\right) / s=\frac{e}{d} Q$. En particulier, $Q$ est dans $\left.\operatorname{Div}^{0}\left(X_{E}\right) \cap \operatorname{Im}_{\operatorname{Div}}^{0}\left(X_{E_{T}}\right) \otimes \mathbb{Q}\right]$ si bien que $Q=\frac{e_{T}}{e} \pi^{*}\left(\pi_{*}(Q)\right)$. On obtient donc une égalité de diviseurs de $X_{E_{T}}$

$$
\frac{\operatorname{div}_{T}\left(\gamma_{0}\right)}{s}=\frac{e_{T}}{d} \pi_{*}(Q)
$$

avec $\pi_{*}(Q) \in \operatorname{Div}^{0}\left(X_{E_{T}}\right)$. C'est ce qu'il fallait pour qu'il existe une algèbre simple centrale $C$ sur $E_{T}$ telle que

$$
D^{o p} \otimes_{F} C \simeq \operatorname{End}\left((W, \psi)^{r}\right) .
$$

Étape 3 : plonger $E$ dans les endomorphismes de $(V, \tau)$. On a obtenu un $D^{o p} \otimes_{\mathbb{F}_{q}} k$ module $V$ muni d'une bijection $\operatorname{id}_{D} \otimes \sigma$-linéaire $\tau$. De plus, l'anneau des endomorphismes de $(V, \tau)$ est égal à $C$. Il reste à construire une bijection id ${ }_{D} \otimes \sigma^{s}$-linéaire $\tau^{\prime}$ qui commute à $\tau$. Il revient au même de construire une action de $\gamma_{0}$ ou ce qui est équivalent de montrer l'existence d'un plongement $E \hookrightarrow C$. D'après le lemme 2 , il suffit de démontrer que $\left[E: E_{T}\right]=e / e_{T}$ divise $d / e_{T}$ et que

$$
\operatorname{inv}(C) \in \frac{e}{d} \operatorname{Div}^{0}\left(X_{E}\right) / \operatorname{Div}^{0}\left(X_{E}\right) .
$$

La première divisibilité est automatique. Par ailleurs, dans $\operatorname{Div}^{0}\left(X_{E}\right) \otimes \mathbb{Q} / \mathbb{Z}$, on a l'égalité

$$
\operatorname{inv}(C)=-\frac{\operatorname{div}_{T}\left(\gamma_{0}\right)}{s}-\operatorname{inv}\left(D^{o p}\right) .
$$


Puisque $E$ peut être plongé dans $D^{o p}$, on a d'après le lemme 3

$$
\operatorname{inv}\left(D^{o p}\right) \in \frac{e}{d} \operatorname{Div}^{0}\left(X_{E}\right) / \operatorname{Div}^{0}\left(X_{E}\right)
$$

Par ailleurs, on a

$$
\frac{\operatorname{div}_{T}\left(\gamma_{0}\right)}{s} \in \frac{e}{d} \operatorname{Div}^{0}\left(X_{E}\right)
$$

d'après le lemme 3. Donc, on peut plonger $E$ dans $C=\operatorname{End}(V, \tau)$ et obtenir une bijection $\operatorname{id}_{D} \otimes k$-linéaire de $V$ commutant à $\tau$. Posons $\tau^{\prime}=\gamma_{0} \tau^{s}$.

Étape 4 : le triplet $\left(V ; \tau, \tau^{\prime}\right)$ est bien un objet de $C\left(T, T^{\prime} ; s\right)$. Soit $n$ un entier assez divisible tel qu'il existe un $D \otimes_{\mathbb{F}_{q}} \mathbb{F}_{q^{n}}$-sous-module de $V^{\prime}$, stable par $\tau$ et $\tau^{\prime}$, tel que $V=$ $V^{\prime} \otimes_{\mathbb{F}_{q^{n}}} k$. Notons $E^{\prime}$ la $F$-sous-algèbre de $\operatorname{End}\left(V^{\prime}\right)$ engendrée par $\left.\tau^{n}\right|_{V^{\prime}}$ et $\left.\tau^{\prime \prime}\right|_{V^{\prime}}$. On a dans $E^{\prime}$ l'égalité

$$
\gamma_{0}^{n}=\left.\left.\tau^{n s}\right|_{V^{\prime}} \tau^{\prime-n}\right|_{V^{\prime}}
$$

Les paires $\left(E, \gamma_{0}^{n}\right)$ et $\left(E^{\prime}, \gamma_{0}^{n}\right)$ sont isomorphes dans la catégorie $\mathcal{E}$ de sorte que le diviseur $\operatorname{div}\left(\gamma_{0}^{n}\right)$ sur $X_{E^{\prime}}$ est supporté par $T \cup T^{\prime}$. Par construction de $\tau$, la partie de $\operatorname{div}\left(\gamma_{0}^{n}\right)$ supportée $\operatorname{par} T$ est $\operatorname{div}\left(\left.\tau^{n s}\right|_{V^{\prime}}\right)$; il s'ensuit que la partie supportée par $T^{\prime}$ est $\operatorname{div}\left(\left.\tau^{\prime-n}\right|_{V^{\prime}}\right)$.

Soit $x \in|X-T|$. Par ce qui précède, l'élément $\left.\tau_{x}^{n s}\right|_{V_{x}^{\prime}}$ est une unité de $E_{x}^{\prime}$. L'application $\operatorname{id}_{D} \otimes \sigma$-linéaire $\left.\tau_{x}\right|_{V_{x}^{\prime}}$ du $D_{x} \otimes_{\mathbb{F}_{q}} \mathbb{F}_{q^{n}}$-module $V_{x}^{\prime}$ a une $n s$-norme $\left.\tau_{x}^{n s}\right|_{V_{x}^{\prime}}$ compacte. D'après Kottwitz, $\left.\tau_{x}\right|_{V_{x}^{\prime}}$ doit fixer un $\mathcal{D}_{x} \widehat{\otimes}_{\mathbb{F}_{q}} \mathbb{F}_{q^{n}}$-réseau de sorte que la paire $\left(V_{x}, \tau_{x}\right)$ est isomorphe à la paire $\left(D_{x} \widehat{\otimes}_{\mathbb{F}_{q}} k, \operatorname{id}_{D_{x}} \widehat{\otimes} \sigma\right)$.

L'assertion sur $\tau^{\prime}$ se démontre de même.

Étape 5 : la classe de conjugaison associée à $\left(V ; \tau, \tau^{\prime}\right)$ est bien $\gamma_{0}$. C'est évident sur la construction de $\tau^{\prime}=\gamma_{0} \tau^{s}$.

On a donc démontré que l'application $\left(V ; \tau, \tau^{\prime}\right) \mapsto \gamma_{0}$ de l'ensemble des classes d'isomorphisme des objets de $C\left(T, T^{\prime} ; s\right)$ sur l'ensemble des classes de conjugaison de $G(F)$ qui sont $\left(T, T^{\prime}\right)$-admissibles et $s$-norme en $T$, est surjective. Nous allons maintenant vérifier qu'elle est injective.

Soit $\gamma_{0}$ une classe de conjugaison de $G(F)$ qui est $\left(T, T^{\prime}\right)$-admissible et $s$-norme en $T$. Soit $E$ la $F$-sous-algèbre de $D$ engendrée par un représentant $\gamma_{0}$ de la classe de conjugaison $\gamma_{0}$.

Soit $\left(V ; \tau, \tau^{\prime}\right)$ un objet de $C\left(T, T^{\prime} ; s\right)$ dont la classe de conjugaison associée dans $G(F)$, est $\gamma_{0}$. Puisque $(V, \tau)$ est irréductible comme $D \otimes_{\mathbb{F}_{q}} k$-module muni d'une bijection id ${ }_{D} \otimes_{\mathbb{F}_{q}} k$ linéaire, en oubliant l'action de $D$, comme $\phi$-espace, $(V, \tau)$ est isotypique. Le $\phi$-espace irréductible $(W, \psi)$, facteur de $(V, \tau)$, est complètement déterminé par $\gamma_{0}$, car la $\phi$-paire qui lui est associée par le théorème 4 de 4.3 est isomorphe dans $\mathcal{E}$ à la $\phi$-paire $\left(E, \operatorname{div}_{T}\left(\gamma_{0}\right)\right)$. Ainsi $(W, \psi)$ est nécessairement le $\phi$-espace irréductible construit dans l'étape 1.

Comme $\phi$-espace, $(V, \tau)$ est donc nécessairement isomorphe à $(W, \psi)^{r}$ où l'entier $r$ est défini comme dans l'étape 2. La structure de $D^{o p}$-module est alors donnée par un homomorphisme d'algèbres

$$
D^{o p} \hookrightarrow \operatorname{End}(W, \psi)^{r} .
$$

Dans l'étape 2, on a démontré que celui-ci existe. Il est de plus unique à automorphisme intérieur près, d'après le théorème de Skolem-Noether.

Il reste à construire une bijection $\operatorname{id}_{D} \otimes \sigma^{s}$-linéaire $\tau^{\prime}$ de $V$ commutant à $\tau$ telle que l'automorphisme $\tau^{s} \tau^{\prime-1}$ est conjugué dans $G\left(F \otimes_{\mathbb{F}_{q}} k\right)$ à $\gamma_{0}$. Il revient au même de plonger

$4^{\text {e }}$ SÉRIE - TOME $39-2006-\mathrm{N}^{\circ} 2$ 
l'algèbre $E=F\left[\gamma_{0}\right]$ dans le commutant $C$ de $D^{o p}$ dans $\operatorname{End}(W, \psi)^{r}$. On a démontré dans l'étape 3 que ce plongement existe. Il est unique à automorphisme intérieur près, de nouveau d'après le théorème de Skolem-Noether.

\subsection{Le groupe des automorphismes de $\left(V ; \tau, \tau^{\prime}\right)$}

Soit $\gamma_{0}$ une classe de conjugaison de $G(F)$ qui est $\left(T, T^{\prime}\right)$-admissible et $s$-norme en $T$. Soit $\left(V ; \tau, \tau^{\prime}\right)$ l'objet de $C\left(T, T^{\prime} ; s\right)$ qui lui correspond et qui est bien défini à isomorphisme près. On va démontrer que le groupe des automorphismes de $\left(V ; \tau, \tau^{\prime}\right)$ est le groupe des $F$-points d'une forme intérieure $J_{\gamma_{0}}$ du centralisateur $G_{\gamma_{0}}$ de $\gamma_{0}$ dans $G$. D'après le principe de Hasse pour les groupes adjoints, la classe d'isomorphisme de cette forme intérieure est complètement déterminée par celle des groupes locaux $J_{\gamma_{0}, v}$, formes intérieures de $G_{\gamma_{0}, v}$ qu'on va décrire explicitement.

L'automorphisme $\gamma=\tau^{s} \tau^{\prime-1}$ de $V$ détermine une classe de conjugaison de $G\left(F \otimes_{\mathbb{F}_{q}} k\right)$ dont on choisit un représentant noté aussi $\gamma$. Soit $G_{\gamma}\left(F \otimes_{\mathbb{F}_{q}} k\right)$ le centralisateur de $\gamma$ dans $G\left(F \otimes_{\mathbb{F}_{q}} k\right)$. Puisque $\tau$ commute avec $\gamma, \tau$ détermine un automorphisme de $G_{\gamma}\left(F \otimes_{\mathbb{F}_{q}} k\right)$. Formons le produit semi-direct

$$
G_{\gamma}\left(F \otimes_{\mathbb{F}_{q}} k\right) \rtimes\langle\tau\rangle .
$$

Il est clair que $\operatorname{Aut}\left(V ; \tau, \tau^{\prime}\right)$ est le groupe des points fixes de $G_{\gamma}\left(F \otimes_{\mathbb{F}_{q}} k\right)$ sous l'action de $\tau$. On veut construire un $F$-groupe $J_{\gamma_{0}}$ dont le groupe des $F$-points est $\operatorname{Aut}\left(V ; \tau, \tau^{\prime}\right)$.

On choisit un représentant $\gamma_{0} \in G(F)$ dans la classe de conjugaison $\gamma_{0}$. Le centralisateur $G_{\gamma_{0}}$ étant défini sur $F$, l'endomorphisme de Frobenius agit comme un automorphisme sur $G_{\gamma_{0}}\left(F \otimes_{\mathbb{F}_{q}} k\right)$. Formons le produit semi-direct

$$
G_{\gamma_{0}}\left(F \otimes_{\mathbb{F}_{q}} k\right) \rtimes\langle\sigma\rangle .
$$

Il est clair que le groupe $G_{\gamma_{0}}(F)$ est le groupe des points fixes de $G_{\gamma_{0}}\left(F \otimes_{\mathbb{F}_{q}} k\right)$ sous l'action de $\sigma$.

LEMME 1. - Pour $r$ assez divisible, on a un isomorphisme de produits semi-directs

$$
G_{\gamma}\left(F \otimes_{\mathbb{F}_{q}} k\right) \rtimes\left\langle\tau^{r}\right\rangle \stackrel{\sim}{\longrightarrow} G_{\gamma_{0}}\left(F \otimes_{\mathbb{F}_{q}} k\right) \rtimes\left\langle\sigma^{r}\right\rangle .
$$

Démonstration. - Prenons une $\mathbb{F}_{q}$-structure de $D^{o p} \otimes_{\mathbb{F}_{q}} k$-module $V$. Pour une extension assez grande de $\mathbb{F}_{q}, \tau$ et $\tau^{\prime}$ vont être définis sur cette extension si bien qu'il va en être de même de $\gamma$. On peut donc supposer $\gamma=\gamma_{0}$.

Soit $E=F[\gamma]$. Puisque l'homomorphisme $E^{\times} \rightarrow \operatorname{Div}^{0}\left(X_{E}\right)$ a un noyau et un conoyau finis, on peut décomposer

$$
\gamma^{r}=\delta^{s} \delta^{\prime-1}
$$

avec $\delta, \delta^{\prime} \in E^{\times}$tels que $\operatorname{div}(\delta)$ soit supporté par $T$, et $\operatorname{div}\left(\delta^{\prime}\right)$ par $T^{\prime}$ en utilisant l'hypothèse que $\gamma$ est $\left(T, T^{\prime}\right)$-admissible et $s$-norme en $T$, pour un entier $r$ assez divisible. L'assertion d'injectivité du théorème 1 de 4.5 montre que les triplets $\left(V ; \tau^{r}, \tau^{\prime r}\right)$ et $\left(V ; \delta \sigma^{r}, \delta^{\prime} \sigma^{r s}\right)$ sont isomorphes car ils correspondent tous les deux à la classe de conjugaison de $\gamma^{r}$. Il ne reste qu'à remarquer que $\delta \in E^{\times}$appartient au centre de $G_{\gamma}\left(F \otimes_{\mathbb{F}_{q}} k\right)$ de sorte que les actions de $\sigma^{r}$ et de $\delta \sigma^{r} \operatorname{sur} G_{\gamma}\left(F \otimes_{\mathbb{F}_{q}} k\right)$ sont les mêmes. La proposition s'en déduit.

Proposition 2. - Soit $\gamma_{0}$ une classe de conjugaison de $G(F)$ qui est $\left(T, T^{\prime}\right)$-admissible et s-norme en $T$. Soit $\left(V ; \tau, \tau^{\prime}\right)$ la classe d'isomorphisme de la catégorie des fibres génériques 
$C\left(T, T^{\prime} ; s\right)$ qui lui correspond par le théorème 1 de 4.5. Il existe une forme intérieure $J_{\gamma_{0}}$ de $G_{\gamma_{0}}$ telle que

$$
J_{\gamma_{0}}(F)=\operatorname{Aut}\left(V ; \tau, \tau^{\prime}\right) .
$$

Démonstration. - Le triplet $\left(V ; \tau, \tau^{\prime}\right)$ induit une paire $(V ; \gamma)$ où on peut voir $V$ comme un $G$ torseur sur $F \otimes_{\mathbb{F}_{q}} k$ et où $\gamma: V \rightarrow V$ est un automorphisme de ce $G$-torseur. L'hypothèse que $\gamma$ et $\gamma_{0}$ soient conjugués dans $G\left(F \otimes_{\mathbb{F}_{q}} k\right)$ implique que la paire $(V, \gamma)$ est isomorphe à $\left(G \otimes_{\mathbb{F}_{q}} k, \gamma_{0}\right)$. L'ensemble des isomorphismes

$$
\mathcal{L}:=\operatorname{Isom}\left(\left(G \otimes_{\mathbb{F}_{q}} k, \gamma_{0}\right),(V, \gamma)\right)
$$

définit donc un $G_{\gamma_{0}}$-torseur sur $F \otimes_{\mathbb{F}_{q}} k$.

Le diagramme commutatif

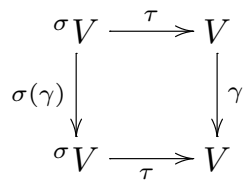

induit un isomorphisme $\tau:{ }^{\sigma} \mathcal{L} \stackrel{\sim}{\longrightarrow}$ et donc une classe dans

$$
\mathrm{H}^{1}\left(\langle\sigma\rangle, G_{\gamma_{0}}(F \otimes k)\right) .
$$

La proposition se ramène à démontrer que l'image de cette classe dans

$$
\mathrm{H}^{1}\left(\langle\sigma\rangle, G_{\gamma_{0}}^{\mathrm{ad}}(F \otimes k)\right)
$$

provient d'un cocycle continu, c'est-à-dire d'un élément de $\mathrm{H}^{1}\left(\widehat{\mathbb{Z}}, G_{\gamma_{0}}^{\text {ad }}(F \otimes k)\right)$, ce qui est le contenu du lemme précédent.

D'après le principe de Hasse pour les groupes adjoints, la forme intérieure $J_{\gamma_{0}}$ de $G_{\gamma_{0}}$ est complètement déterminée par les formes locales $J_{\gamma_{0}, v}$ de $G_{\gamma_{0}, v}$ qu'on peut décrire facilement à partir de $\left(V ; \tau, \tau^{\prime}\right)$.

- Soit $v$ une place de $X$ en dehors de $T$. Par hypothèse la paire $\left(V_{v}, \tau_{v}\right)$ est isomorphe à $\left(D_{v}^{o p} \widehat{\otimes}_{\mathbb{F}_{q}} k, \mathrm{id}_{D_{v}} \widehat{\otimes} \sigma\right)$. Les vecteurs fixes sous $\tau_{v}$ forment donc un $D_{v}^{o p}$-module libre $V_{x}^{\tau_{v}=1}$. Puisque $\tau_{v}^{\prime}$ commute à $\tau$, il laisse stable $V_{v}^{\tau_{v}=1}$ et sa restriction à $V_{v}^{\tau_{v}=1}$ est une bijection $D_{x}^{o p}$-linéaire dont la classe de conjugaison est $\gamma_{0}$. Un automorphisme $D_{v}^{o p} \widehat{\otimes}_{\mathbb{F}_{q}} k$ linéaire de $V_{v}$ qui commute à $\tau_{v}$ laisse stable $V_{v}^{\tau_{v}=1}$. Si de plus, il commute à $\tau^{\prime}$, sa restriction à $V_{v}^{\tau_{v}=1}$ doit commuter à la restriction de $\tau_{v}^{\prime}$. Cela définit une bijection de $J_{\gamma_{0}}\left(F_{v}\right)$ avec le centralisateur de $\gamma_{0}$ dans $G\left(F_{x}\right)$.

- Pour une place $x \in|T|$, on a nécessairement $x \notin\left|T^{\prime}\right|$, de sorte que la paire $\left(V_{x}, \tau_{s}^{\prime}\right)$ est isomorphe à

$$
\left(\left(D_{x} \widehat{\otimes}_{\mathbb{F}_{q}} \mathbb{F}_{q^{s}}\right) \widehat{\otimes}_{\mathbb{F}_{q} s} k, \mathrm{id}_{D_{x} \widehat{\otimes}_{\mathbb{F}_{q}} \mathbb{F}_{q^{s}}} \widehat{\otimes} \sigma^{s}\right) .
$$

Les vecteurs fixes sous $\tau_{x}^{\prime}$ forment un $D_{x} \widehat{\otimes}_{\mathbb{F}_{q}} \mathbb{F}_{q^{s}}$-module $V_{x}^{\tau_{x}^{\prime}=1}$ libre de rang 1 . Puisque $\tau_{x}$ commute à $\tau_{x}^{\prime}, \tau_{x}$ définit une bijection $\sigma$-linéaire de $V_{x}^{\tau_{x}^{\prime}=1}$ dans lui-même et donc une classe de $\sigma$-conjugaison $\delta_{x}$ du groupe $G\left(F_{x} \widehat{\otimes}_{\mathbb{F}_{q}} \mathbb{F}_{q^{s}}\right)$. On déduit de la relation $\gamma_{0}=\tau^{s} \tau^{\prime-1}$ que la $s$-norme de $\delta_{x}$ est égale à $\gamma_{0}$. Le groupe $J_{\gamma_{0}}\left(F_{x}\right)$ est alors le centralisateur tordu de $\delta_{x}$. 
COROLlaire 3. - Si en toutes les places $v$ de $F, J_{\gamma_{0}, v}$ est isomorphe à $G_{\gamma_{0}, v}$ alors $J_{\gamma_{0}}$ est isomorphe à $G_{\gamma_{0}}$.

\subsection{Compter les réseaux}

On est maintenant en mesure de démontrer une formule de comptage pour la catégorie $\mathfrak{C}_{\alpha_{T}, \beta_{T^{\prime}}}^{I}\left(T, T^{\prime} ; s\right)$ où $\alpha_{T}$ est une fonction $\alpha_{T}:\left|T \otimes_{\mathbb{F}_{q}} \mathbb{F}_{q^{s}}\right| \rightarrow \mathbb{Z}_{+}^{d}$ et où $\beta_{T^{\prime}}$ est une fonction qui associe à toute place $v \in\left|T^{\prime}\right|$ une double classe $\beta_{v} \in K_{v}^{I} \backslash G\left(F_{v}\right) / K_{v}^{I}$.

On a construit un foncteur

$$
\mathfrak{C}\left(T, T^{\prime} ; s\right) \rightarrow C\left(T, T^{\prime} ; s\right)
$$

qui associe à un triplet $\left(\mathcal{V} ; t, t^{\prime} ; \iota\right)$ sa fibre générique $\left(V ; \tau, \tau^{\prime}\right)$. On a ensuite démontré que l'application qui associe à une classe d'isomorphisme d'objets $\left(V ; \tau, \tau^{\prime}\right)$ de $C\left(T, T^{\prime} ; s\right)$, l'unique classe de conjugaison $\gamma_{0}$ de $G(F)$, conjuguée dans $G\left(F \otimes_{\mathbb{F}_{q}} k\right)$ à $\gamma=\tau^{s}{\tau^{\prime}}^{-1}$, induit une bijection de l'ensemble des classes d'isomorphisme de $C\left(T, T^{\prime} ; s\right)$ sur l'ensemble des classes de conjugaison de $G(F)$ qui sont $\left(T, T^{\prime}\right)$-admissibles et $s$-normes en $T$. On sait aussi qu'il existe une forme intérieure $J_{\gamma_{0}}$ du centralisateur $G_{\gamma_{0}}$ telle que $J_{\gamma_{0}}(F)$ est le groupe des automorphismes de $\left(V ; \tau, \tau^{\prime}\right)$ et, pour toute place $x$ de $F, J_{\gamma_{0}}\left(F_{x}\right)$ est le groupe des automorphismes de $\left(V_{x} ; \tau_{x}, \tau_{x}^{\prime}\right)$.

THÉORÈME 1. - Le nombre pondéré des classes d'isomorphisme

$$
\# \mathfrak{C}_{\alpha_{T}, \beta_{T^{\prime}}}^{I}\left(T, T^{\prime} ; s\right)=\sum_{\left(\mathcal{V} ; t, t^{\prime} ; \iota\right)} \frac{1}{\# \operatorname{Isom}\left(\mathcal{V} ; t, t^{\prime}\right)}
$$

où $\left(\mathcal{V} ; t, t^{\prime} ; \iota\right)$ parcourt un ensemble de représentants de classes d'isomorphisme de $\mathfrak{C}_{\alpha_{T}, \beta_{T^{\prime}}}^{I}\left(\bar{T}, \bar{T}^{\prime} ; s\right)$, est fini et est égal à

$$
\sum_{\left(\gamma_{0} ; \delta_{T}\right)} \operatorname{vol}\left(a^{\mathbb{Z}} J_{\gamma_{0}, \delta_{T}}(F) \backslash J_{\gamma_{0}, \delta_{T}}\left(\mathbb{A}_{F}\right)\right) \prod_{v \notin|T|} \mathbf{O}_{\gamma_{0}}\left(\phi_{\beta_{v}}\right) \prod_{x \in|T|} \mathbf{T O}_{\delta_{x}}\left(\phi_{\alpha_{x}}\right)
$$

Ici :

- la somme est étendue sur l'ensemble des paires $\left(\gamma_{0}, \delta_{T}\right)$ formées d'une classe de conjugaison $\gamma_{0}$ de $D^{\times}$qui est $\left(T, T^{\prime}\right)$-admissible et d'une collection $\delta_{T}=\left(\delta_{x}\right)_{x \in T}$ de classe de $\sigma$-conjugaison $\delta_{x}$ de $G\left(F_{x} \otimes_{\mathbb{F}_{q}} \mathbb{F}_{q^{s}}\right)$ dont la norme est $\gamma_{0}$;

- le groupe $J_{\gamma_{0}, \delta_{T}}$, défini dans 4.6, est la forme intérieure de $G_{\gamma_{0}}$ dont les $F$-points forment le groupe des automorphismes du triplet $\left(V ; \tau, \tau^{\prime}\right)$ correspondant à la classe de conjugaison $\gamma_{0}$ et dont les $F_{x}$-points forment le groupe des automorphismes $\operatorname{Aut}\left(V_{x} ; \tau_{x}, \tau_{x}^{\prime}\right)$;

- pour toute place $x \in|T|$, on définit

$$
\phi_{\alpha_{x}}=\bigotimes_{y \in\left|x \otimes_{\mathbb{F}_{q}} \mathbb{F}_{q^{s}}\right|} \phi_{\alpha_{T}(y)} \in \bigotimes_{y \in\left|x \otimes_{\mathbb{F}_{q}} \mathbb{F}_{q^{s}}\right|} \mathcal{H}_{y}=\mathcal{H}\left(G\left(F_{x} \otimes_{\mathbb{F}_{q}} \mathbb{F}_{q^{s}}\right)\right) ;
$$

- pour toute place $v \in\left|T^{\prime}\right|$, on définit $\phi_{\beta_{v}} \in \mathcal{H}_{v}^{I}$ comme la fonction caractéristique de la double classe $\beta_{v} \in K_{v}^{I} \backslash G\left(F_{v}\right) / K_{v}^{I}$. En une place $v \notin|T| \cup\left|T^{\prime}\right|$, la fonction $\phi_{\beta_{v}}$ désigne la fonction caractéristique de $G\left(\mathcal{O}_{v}\right)$ dans $G\left(F_{v}\right)$. 
Démonstration. - Soit $\gamma_{0}$ une classe de conjugaison de $G(F)$ qui est $\left(T, T^{\prime}\right)$-admissible et $s$-norme en $T$. Soit $\left(V ; \tau, \tau^{\prime}\right)$ un objet de $C\left(T, T^{\prime} ; s\right)$ qui correspond à $\gamma_{0}$. Donner un objet

$$
\left(\mathcal{V} ; t, t^{\prime} ; \iota\right) \in \mathfrak{C}_{\alpha_{T}, \beta_{T^{\prime}}}^{I}\left(T, T^{\prime} ; s\right)
$$

de fibre générique $\left(V ; \tau, \tau^{\prime}\right)$ revient à donner en toutes les places $v \in|X|$, un $\mathcal{D}_{v} \widehat{\otimes}_{\mathbb{F}_{q}} k$-réseau $\mathcal{V}_{v}$, plus une $I$-structure de niveau tels que

- en une place $v \notin|T|, \mathcal{V}_{v}$ est fixe par $\tau$,

- en une place $v \notin\left|T^{\prime}\right|, \mathcal{V}_{v}$ est fixe par $\tau^{\prime}$,

- en une place $x \in|T|$, la position relative entre $\mathcal{V}_{x}$ et $\tau\left(\mathcal{V}_{x}\right)$ est donnée par $\alpha_{x}$,

- en une place $v \in\left|T^{\prime}\right|$, donc hors de $T$, les descentes à l'aide de $\tau$ de $F_{v} \widehat{\otimes}_{\mathbb{F}_{q}} k$ à $F_{v}$ de $\mathcal{V}_{v}$ et $\tau^{\prime}\left(\mathcal{V}_{v}\right)$, munis de leurs structures de niveaux, sont en position relative $\beta_{v}$.

Il s'agit de compter le nombre de ces réseaux modulo la relation d'équivalence induite par la multiplication scalaire par l'idèle $a \in \mathbb{A}_{F}^{\times}$et par l'action de

$$
\operatorname{Aut}\left(V ; \tau, \tau^{\prime}\right)=J_{\gamma_{0}, \delta_{T}}(F)
$$

Que ce nombre soit égal à l'expression intégrale dans l'énoncé du théorème résulte de la définition même des intégrales orbitales.

\section{L'identité de changement de base}

On va donner la démonstration du théorème 1 de 3.3 dans les paragraphes 5.1-5.4 de ce chapitre. On va en tirer une nouvelle démonstration du lemme fondamental pour le changement de base en 5.7.

Rappelons qu'on a supposé que $D$ a plus de $d^{2} r\|\lambda\|$ places totalement ramifiées. Ceci implique la propreté des deux morphismes caractéristiques $c_{\lambda}^{I}$ et $c_{r . \lambda}^{I}$ d'après 1.6.

\subsection{Théorème de densité de Chebotarev}

Soit $U$ l'ouvert de $\left(X^{\prime}-I\right)^{r}$ complémentaire de la réunion des diagonales. Le groupe symétrique $\mathfrak{S}_{r}$ agissant $\operatorname{sur}\left(X^{\prime}-I\right)^{r}$ laisse stable $U$, et son action y est libre. En particulier, l'action sur $U$ du sous-groupe cyclique $\langle\tau\rangle$ engendré par la permutation cyclique $\tau$, est libre. Considérons le quotient $U_{\langle\tau\rangle}$ de $U$ par l'action de $\langle\tau\rangle$. Ses $k$-points sont les orbites de $\langle\tau\rangle$ dans $U(k)$. Pour tout point géométrique $u \in U(k)$, on note $[u] \in U_{\langle\tau\rangle}(k)$ son orbite sous l'action de $\langle\tau\rangle$.

Choisissons un point géométrique $v$ de $U$. Le revêtement galoisien $U \rightarrow U_{\langle\tau\rangle}$ induit une suite exacte de groupes fondamentaux

$$
1 \rightarrow \pi_{1}(U, v) \rightarrow \pi_{1}\left(U_{\langle\tau\rangle},[v]\right) \rightarrow\langle\tau\rangle \rightarrow 1
$$

Cette suite n'est pas nécessairement scindable.

Par la descente, la catégorie des systèmes locaux sur $U$ munis d'une action compatible de $\langle\tau\rangle$ est équivalente à la catégorie des systèmes locaux sur $U_{\langle\tau\rangle}$ et donc équivalente à la catégorie des représentations continues du groupe $\pi_{1}\left(U_{\langle\tau\rangle},[v]\right)$.

Comme dans 3.1, fixons un point $x \in\left(X^{\prime}-I\right)(k)$ et notons $x^{r} \in\left(X^{\prime}-I\right)(k)$ le point diagonal de coordonnées $x$. En prenant la fibre en $x^{r}$, la catégorie des systèmes locaux sur $\left(X^{\prime}-I\right)^{r}$ munis d'un action compatible de $\langle\tau\rangle$ est équivalente à la catégorie des représentations continues du produit semi-direct $\pi_{1}\left(\left(X^{\prime}-I\right)^{r}, x^{r}\right) \rtimes\langle\tau\rangle$.

$4^{\text {e }}$ SÉRIE - TOME $39-2006-\mathrm{N}^{\circ} 2$ 
Le foncteur de restriction de $\left(X^{\prime}-I\right)^{r}$ à $U$ fournit un homomorphisme de groupes

$$
\pi_{1}\left(U_{\langle\tau\rangle},[v]\right) \rightarrow \pi_{1}\left(\left(X^{\prime}-I\right)^{r}, x^{r}\right) \rtimes\langle\tau\rangle
$$

bien déterminé à un automorphisme intérieur près. Le foncteur de restriction étant fidèle, l'homomorphisme qui s'en déduit est surjectif. Il est de plus compatible avec la projection sur le groupe cyclique $\langle\tau\rangle$. Le choix de ce chemin de $v$ à $x^{r}$ nous permet de reformuler le théorème 1 de 3.3 comme suit. Pour tout $g \in \pi_{1}\left(U_{\langle\tau\rangle}, v\right)$ dont l'image dans le groupe cyclique $\langle\tau\rangle$ est $\tau$, pour tout $f \in \mathcal{H}^{I}$, on a l'égalité

$$
\operatorname{Tr}\left((1 \otimes \cdots \otimes 1 \otimes f) g,\left[A_{[v]}\right]\right)=\operatorname{Tr}\left(f g,\left[B_{[v]}\right]\right) .
$$

Un point fermé de $U_{\langle\tau\rangle}$ de corps résiduel $\mathbb{F}_{q^{s}}$ est une orbite $[u]$ telle que $\sigma^{s}$ soit la plus petite puissance de $\sigma$ laissant stable $[u]$. Soit $u \in U(k)$ un point géométrique de $U$ tel que $[u]$ soit un point fermé de $U_{\langle\tau\rangle}$ de corps résiduel $\mathbb{F}_{q^{s}}$. Il existe un unique $j \in \mathbb{Z} / r \mathbb{Z}$ tel que

$$
\sigma^{s}(u)=\tau^{j}(u) .
$$

Puisque les opérateurs $\sigma$ et $\tau$ commutent, on a $\sigma^{s}\left(u^{\prime}\right)=\tau^{j}\left(u^{\prime}\right)$ pour tout $u^{\prime}$ dans l'orbite $[u]$. Le point fermé $[u]$ de $U_{\langle\tau\rangle}$ définit une classe de conjugaison $\operatorname{Frob}_{[u]}$ de $\pi_{1}\left(U_{\langle\tau\rangle},[v]\right)$ dont l'image dans $\langle\tau\rangle$ est exactement $\tau^{j}$.

Grâce au théorème de Chebotarev, pour démontrer le théorème 1 de 3.3 , il suffit de démontrer que pour tout point fermé $[u]$ de $U_{\langle\tau\rangle}$ tel que la classe de conjugaison Frob ${ }_{[u]} \in \pi_{1}\left(U_{\langle\tau\rangle}\right)$ associée ait l'image $\tau$ dans $\langle\tau\rangle$ et pour tout opérateur de Hecke $f \in \mathcal{H}^{I}$, on a l'égalité

$$
\operatorname{Tr}\left((1 \otimes \cdots \otimes 1 \otimes f) \times \operatorname{Frob}_{[u]},\left[A_{[u]}\right]\right)=\operatorname{Tr}\left(f \times \operatorname{Frob}_{[u]},\left[B_{[u]}\right]\right) .
$$

DÉfINITION 1. - Un point fermé $[u]$ de $U_{\langle\tau\rangle}$ est dit cyclique si l'image de la classe de conjugaison $\operatorname{Frob}_{[u]} \subset \pi_{1}\left(U_{\langle\tau\rangle}\right)$ dans $\langle\tau\rangle$ est le générateur $\tau$.

\subsection{Description des points cycliques}

Proposition 1. - Soient $u=\left(u_{i}\right) \in U(k),[u] \in U_{\langle\tau\rangle}(k)$ son image dans $U_{\langle\tau\rangle}$. Supposons que le point fermé de $U_{\langle\tau\rangle}$ supportant $[u]$ soit de degré s et soit cyclique. Alors, il existe un unique point fermé $x \in\left|X^{\prime}\right|$ de degré rs supportant $\left\{u_{1}, \ldots, u_{r}\right\}$ et il existe un unique point fermé $y \in\left|X^{\prime} \otimes_{\mathbb{F}_{q}} \mathbb{F}_{q^{s}}\right|$ au-dessus de x tel que $y \otimes_{\mathbb{F}_{q^{s}}} k=\left\{u_{1}, \ldots, u_{r}\right\}$.

Démonstration. - Puisque $[u]$ est un point cyclique de corps résiduel $\mathbb{F}_{q^{s}}$, on a

$$
\sigma^{s}\left(u_{i}\right)=u_{i+1} .
$$

L'ensemble $\left\{u_{1}, \ldots, u_{r}\right\}$ définit donc une orbite de $\left\langle\sigma^{s}\right\rangle$ dans $X^{\prime}(k)$ de sorte qu'il existe un unique point fermé $y \in\left|X^{\prime} \otimes_{\mathbb{F}_{q}} \mathbb{F}_{q^{s}}\right|$ tel que $y \otimes_{\mathbb{F}_{q^{s}}} k=\left\{u_{1}, \ldots, u_{r}\right\}$. Puisque $u_{1}, \ldots, u_{r}$ sont deux à deux distincts, le corps résiduel de $X^{\prime} \otimes_{\mathbb{F}_{q}} \mathbb{F}_{q^{s}}$ est nécessairement $\mathbb{F}_{q^{r s}}$.

Soit $x$ le point fermé de $X$ en-dessous de $y$. Il reste à démontrer que le corps résiduel de $X$ en $x$ est aussi $\mathbb{F}_{q^{r s}}$. Supposons qu'il y a $s^{\prime}$ points fermés de $X \otimes_{\mathbb{F}_{q}} \mathbb{F}_{q^{s}}$ au-dessus de $x$. L'extension $F \otimes_{\mathbb{F}_{q}} \mathbb{F}_{q^{s}}$ étant de degré $s$, le degré de décomposition de la place $x$ pour cette extension est $s^{\prime}$ si bien que le degré d'inertie est $s^{\prime \prime}$ avec $s=s^{\prime} s^{\prime \prime}$. En particulier, le corps résiduel de $x$ est $\mathbb{F}_{q^{r s^{\prime}}}$. 
Le groupe $\langle\sigma\rangle$ agit transitivement sur l'ensemble des places de $F \otimes_{\mathbb{F}_{q}} \mathbb{F}_{q^{s}}$ au-dessus de $x$ et le stabilisateur de chacune de ces places est $\sigma^{s^{\prime}}$. On a donc

$$
\mathbb{F}_{q^{r s^{\prime}}} \otimes_{\mathbb{F}_{q^{s^{\prime}}}} \mathbb{F}_{q^{s}}=\mathbb{F}_{q^{r s}}
$$

ce qui implique que $r$ et $s^{\prime \prime}$ sont premiers entre eux.

De plus, le point fermé $y$ étant fixe par $\sigma^{s^{\prime}}$, l'opérateur $\sigma^{s^{\prime}}$ permute l'ensemble des points géométriques $\left\{u_{1}, \ldots, u_{r}\right\}$ au-dessus de $y$ et consiste donc en une certaine permutation $\tau^{\prime}$ de l'ensemble $\{1, \ldots, r\}$ avec $\tau^{\prime s^{\prime \prime}}=\tau$. Puisque $s^{\prime \prime}$ est premier à $r$, il existe $r^{\prime \prime} \in \mathbb{N}$ tel que $s^{\prime \prime} r^{\prime \prime}$ soit congru à 1 modulo $r$. On en déduit que $\tau^{\prime}=\tau^{r^{\prime \prime}}$ donc $\tau^{\prime}$ appartient à $\langle\tau\rangle$. Donc l'orbite $\left[u_{i}\right]$ de $\left(u_{i}\right)$ sous l'action $\langle\tau\rangle$ est stable par $\sigma^{s^{\prime}}$.

Puisque $s$ est par hypothèse, le plus petit entier ayant cette propriété, $s^{\prime}$ est nécessairement égal à $s$. On en déduit que le corps résiduel de $X$ en $x$ est bien $\mathbb{F}_{q^{r s}}$.

\subsection{Calcul des traces sur $[B]$ aux points cycliques}

Soit $\left(u_{i}\right)=u \in U(k)$ dont l'image $[u]$ dans $U_{\langle\tau\rangle}$ est un point cyclique de degré $s$. D'après 5.2, $u_{1}, u_{2}, \ldots, u_{r}$ sont supportés par une place $x$ de $X$ de degré $r s$ et sont les points géométriques au-dessus d'un point fermé $y$ de $X \otimes_{\mathbb{F}_{q}} \mathbb{F}_{q^{s}}$ au-dessus de $x$. On va prendre $T=\{x\}$.

La fibre du morphisme $c_{r . \lambda}^{I}: \mathcal{S}_{r . \lambda}^{I} \rightarrow\left(X^{\prime}-I\right)^{r}$ au-dessus de $u$ est l'ensemble

$$
\mathcal{S}_{r . \lambda}^{I}(u)(k)=\left\{t:{ }^{\sigma} \mathcal{V}^{\bar{T}} \stackrel{\sim}{\longrightarrow} \mathcal{V}^{\bar{T}} \mid \operatorname{inv}(t) \leqslant \sum_{i=1}^{r} \lambda u_{i}\right\} / a^{\mathbb{Z}},
$$

où $\mathcal{V} \in \mathcal{D}-\operatorname{Fib}(k)$ et où $\bar{T}=T \otimes_{\mathbb{F}_{q}} k$ contient $r s$ points parmi lesquels $\left\{u_{1}, \ldots, u_{r}\right\} \subset \bar{T}$. Le diviseur $u_{1}+\cdots+u_{r}$ est fixe par $\sigma^{s}$ de sorte qu'on a une $\mathbb{F}_{q^{s}}$-structure sur $\mathcal{S}_{r . \lambda}^{I}(u)$. On peut appliquer la formule de trace de Grothendieck-Lefschetz pour obtenir la formule suivante.

Proposition 1. - Pour tout fermé $T^{\prime}$ de $X$, disjoint de $T$, et pour toute fonction $\beta_{T^{\prime}}$ qui à toute place $v \in\left|T^{\prime}\right|$ associe une double classe

$$
\beta_{v} \in K_{v}^{I} \backslash G\left(F_{v}\right) / K_{v}^{I},
$$

on a l'égalité

$$
\begin{aligned}
& \operatorname{Tr}\left(\sigma^{s} \circ \Phi_{\beta_{T^{\prime}}}, \operatorname{R\Gamma }\left(\mathcal{S}_{r . \lambda}^{I}(u), \mathcal{F}_{r . \lambda}\right)\right) \\
& \quad=\sum_{z \in \operatorname{Fix}\left(\sigma^{s} \circ \Phi_{\beta_{T^{\prime}}}\right)} \# \operatorname{Aut}(z)^{-1} \operatorname{Tr}\left(\sigma^{s} \circ \Phi_{\beta_{T^{\prime}}},\left(\mathcal{F}_{r . \lambda}\right)_{z}\right) .
\end{aligned}
$$

Par définition, un objet de la catégorie des points fixes de la correspondance $\sigma^{s} \circ \Phi_{\beta_{T^{\prime}}}$ dans $\mathcal{S}_{r . \lambda}^{I}(u)$ consiste en un chtouca $(\mathcal{V}, t, \iota)$ d'invariant de Hodge $\operatorname{inv}(t) \leqslant \sum_{i=1}^{r} \lambda u_{i}$ et muni d'une $I$-structure de niveau $\iota$, plus d'une $T^{\prime}$-modification $t^{\prime}: \sigma^{s} \mathcal{V}^{T^{\prime}} \stackrel{\sim}{\longrightarrow} \mathcal{V}^{T^{\prime}}$, commutant à $t$ et qui en une place $v \in T^{\prime}$ ayant le type $\beta_{v}$. Notons que les $u_{i}$ sont les points géométriques au-dessus d'un seul point fermé $y \in x \otimes_{\mathbb{F}_{q}} \mathbb{F}_{q^{s}}$ d'après 5.2.

Proposition 2. - Soit $\left(\mathcal{V}, t, t^{\prime}, \iota\right) \in \operatorname{Fix}\left(\sigma^{s} \circ \Phi_{\beta_{T^{\prime}}}, \mathcal{S}_{r . \lambda}^{I}(u)\right)$; alors il existe $\alpha \in \mathbb{Z}_{+}^{d}$ avec $\alpha \leqslant \lambda$ tel qu'on a

$$
\operatorname{inv}(t)=\sum_{i=1}^{r} \alpha u_{i}
$$

$4^{\mathrm{e}}$ SÉRIE - TOME $39-2006-\mathrm{N}^{\circ} 2$ 
De plus, on a

$$
\operatorname{Tr}\left(\sigma^{s} \circ \Phi_{\beta_{T^{\prime}}},\left(\mathcal{F}_{r . \lambda}\right)_{\left(\mathcal{V} ; t, t^{\prime}\right)}\right)=P_{\lambda, \alpha}\left(q^{r s}\right)
$$

où $P_{\lambda, \alpha}\left(q^{r s}\right)$ est définie comme la trace de $\sigma_{q^{r s}}$ sur la fibre de $\mathcal{A}_{\lambda, u_{i}}$ au-dessus du point $\alpha\left(u_{i}\right)$ de $\mathcal{Q}_{\lambda}\left(u_{i}\right)$, correspondant au copoids $\alpha$ et pour n'importe quel $i=1, \ldots, r$.

Démonstration. - Puisque $\sigma^{s}\left(u_{i}\right)=u_{i+1}$ pour tous $i=1, \ldots, r$, on a une $\mathbb{F}_{q^{s}}$-structure de la fibre $\mathcal{Q}_{r . \lambda}(u)=\prod_{i=1}^{n} \mathcal{Q}_{\lambda, u_{i}}$ de $\mathcal{Q}_{r . \lambda}$ au-dessus de $(u)$, donnée par $\sigma^{s}\left(Q_{j}^{i}\right)=\left(Q_{j}^{\prime i}\right)$ avec $Q^{\prime i}{ }_{j}=Q_{j}^{i+1}$. Le morphisme

$$
f_{r . \lambda}: \mathcal{S}_{r . \lambda}^{I}(u) \rightarrow \mathcal{Q}_{r . \lambda}(u)
$$

est compatible aux $\mathbb{F}_{q^{s}}$-structures. Un point $\left(\mathcal{V} ; t, t^{\prime}\right)$ fixe par $\sigma^{s} \circ \Phi_{\beta_{T^{\prime}}}$ doit nécessairement s'envoyer sur un point $Q$ de $\mathcal{Q}_{r . \lambda}(u)$ fixe par $\sigma^{s}$. On a aussi

$$
\operatorname{Tr}\left(\sigma^{s} \circ \Phi_{\beta_{T^{\prime}}},\left(\mathcal{F}_{r . \lambda}\right)_{\left(\mathcal{V} ; t, t^{\prime}\right.}\right)=\operatorname{Tr}\left(\sigma^{s},\left(\mathcal{A}_{r . \lambda}\right)_{Q}\right) .
$$

Il est clair que la $\mathbb{F}_{q^{s}}$-champ $\prod_{i=1}^{r} \mathcal{Q}_{\lambda, u_{i}}$ est la restriction de scalaires à la Weil du $\mathbb{F}_{q^{r s}}$-champ $\mathcal{Q}_{\lambda, u_{i}}$ pour n'importe quel $i$. Le $\mathbb{F}_{q^{s}}$-point $Q$ de $\prod_{i=1}^{r} \mathcal{Q}_{\lambda, u_{i}}$ correspond donc à un $\mathbb{F}_{q^{r s}}$-point $Q^{\prime}$ de $\mathcal{Q}_{\lambda, u_{i}}$. On a alors

$$
\operatorname{Tr}\left(\sigma^{s},\left(\mathcal{A}_{r \cdot \underline{\lambda}}\right)_{Q}\right)=\operatorname{Tr}\left(\sigma^{r s},\left(\mathcal{A}_{\underline{\lambda}}\right)_{Q^{\prime}}\right) .
$$

Il s'agit là d'une propriété complètement générale de la restriction à la Weil dont voici l'énoncé précis. Sa démonstration se ramène essentiellement à la formule de Saito-Shintani dans 3.3.

Proposition 3. - Soient $Z$ un schéma défini sur $\mathbb{F}_{q^{s}}$ et $\mathcal{G}$ un complexe de $\mathbb{Q}_{\ell}$-faisceaux sur $Z$. Soient $Z^{\prime}=\mathrm{R}_{\mathbb{F}_{q^{s}} / \mathbb{F}_{q}} Z$ et $\mathcal{G}^{\prime}=\mathrm{R}_{\mathbb{F}_{q} / \mathbb{F}_{q}} \mathcal{G}$ les restrictions de scalaires à la Weil de $Z$ et de $\mathcal{G}$. Soient $z \in Z\left(\mathbb{F}_{q^{s}}\right)$ un $\mathbb{F}_{q^{s}}$-point de $X$ et $z^{\prime}$ le $\mathbb{F}_{q}$-point de $Z^{\prime}$ qui lui correspond. Alors, on a

$$
\operatorname{Tr}\left(\sigma^{s}, \mathcal{F}_{z}\right)=\operatorname{Tr}\left(\sigma, \mathcal{F}_{z^{\prime}}^{\prime}\right)
$$

COROLlaire 4. - On a la formule

$$
\operatorname{Tr}\left(\sigma^{s} \circ \Phi_{\beta_{T^{\prime}}}, \operatorname{R} \Gamma\left(\mathcal{S}_{r . \lambda}^{I}(u), \mathcal{F}_{r . \lambda}\right)\right)=\sum_{\alpha \leqslant \lambda} P_{\lambda, \alpha}\left(q^{r s}\right) \# \mathfrak{C}_{\alpha_{y}, \beta_{T^{\prime}}}^{I}\left(T, T^{\prime} ; s\right) .
$$

où $\alpha_{y}$ est la fonction $\left|T \otimes_{\mathbb{F}_{q}} \mathbb{F}_{q^{s}}\right| \rightarrow \mathbb{Z}_{+}^{d}$ qui prend la valeur $\alpha$ en $y$ et 0 sur les $s-1$ autres points de $\left|T \otimes_{\mathbb{F}_{q}} \mathbb{F}_{q^{s}}\right|$.

En appliquant le théorème 1 de 4.7, et en tenant compte du fait que l'extension $F \otimes_{\mathbb{F}_{q}} \mathbb{F}_{q^{s}}$ de $F$ est totalement décomposée en $x$, on obtient la formule suivante pour le cardinal $\# \mathfrak{C}_{\alpha_{y}, \beta_{T^{\prime}}}^{I}\left(T, T^{\prime} ; s\right)$

$$
\# \mathfrak{C}_{\alpha_{y}, \beta_{T^{\prime}}}^{I}\left(T, T^{\prime} ; s\right)=\sum_{\gamma_{0}} \operatorname{vol}\left(G_{\gamma_{0}}(F) a^{\mathbb{Z}} \backslash G_{\gamma_{0}}\left(\mathbb{A}_{F}\right)\right) \prod_{v \neq x} \mathbf{O}_{\gamma_{0}}\left(\phi_{\beta_{v}}\right) \mathbf{O}_{\gamma_{0}}\left(\phi_{\alpha_{x}}\right)
$$

où $\phi_{\alpha_{x}} \in \mathcal{H}\left(G\left(F_{x}\right)\right)$ est la fonction caractéristique de la $G\left(\mathcal{O}_{x}\right)$-double classe correspondant à $\alpha$. 
COROLlaire 5. - On a la formule

$$
\begin{aligned}
& \operatorname{Tr}\left(\sigma^{s} \circ \Phi_{\beta_{T^{\prime}}}, \operatorname{R} \Gamma\left(\mathcal{S}_{r . \lambda}^{I}(u), \mathcal{F}_{r . \lambda}\right)\right) \\
& \quad=\sum_{\gamma_{0}} \operatorname{vol}\left(a^{\mathbb{Z}} G_{\gamma_{0}}(F) \backslash G_{\gamma_{0}}\left(\mathbb{A}_{F}\right)\right) \prod_{v \neq x} \mathbf{O}_{\gamma_{0}}\left(\phi_{\beta_{v}}\right) \mathbf{O}_{\gamma_{0}}\left(\psi_{\lambda_{x}}\right) .
\end{aligned}
$$

En effet, on a $\psi_{\lambda_{x}}=\sum_{\alpha \leqslant \lambda} P_{\lambda, \alpha}\left(q^{r s}\right) \phi_{\alpha_{x}}$ car le point $x$ est de degré $r s$.

\subsection{Calcul des traces sur $[A]$ aux points cycliques}

Prenons le même point $u=\left(u_{1}, \ldots, u_{r}\right)$ de $U$ comme dans 5.3 dont l'image $[u]$ dans $U_{\langle\tau\rangle}$ définit un point cyclique de degré $s$. Les points $u_{1}, \ldots, u_{r}$ sont supportés par un point fermé $y$ de $X \otimes_{\mathbb{F}_{q}} \mathbb{F}_{q^{s}}$ de degré $r s$ sur $\mathbb{F}_{q}$. Quant à $y$, il est au-dessus d'un point fermé $x$ de $X$ de degré rs aussi. On va noter $T=\{x\}$.

La fibre du morphisme $\left(c_{\lambda}^{I}\right)^{r}:\left(\mathcal{S}_{\lambda}^{I}\right)^{r} \rightarrow\left(X^{\prime}-I\right)^{r}$ au-dessus de $u$ est munie d'une $\mathbb{F}_{q^{s}}$ structure donnée par

$$
\sigma^{s}\left(\widetilde{\mathcal{V}}_{1}, \ldots, \widetilde{\mathcal{V}}_{r}\right)=\left(\sigma^{s}\left(\widetilde{\mathcal{V}}_{r}\right), \sigma^{s}\left(\widetilde{\mathcal{V}}_{1}\right), \ldots, \sigma^{s}\left(\widetilde{\mathcal{V}}_{r-1}\right)\right)
$$

avec $\widetilde{\mathcal{V}}_{i} \in \mathcal{S}_{\lambda}^{I}\left(u_{i}\right)$. On peut relever cette $\mathbb{F}_{q^{s}}$-structure à la restriction de $\mathcal{F}_{\lambda}^{\bigotimes r}$ à cette fibre.

Soient $T^{\prime}$ et $\beta_{T^{\prime}}$ comme dans le paragraphe précédent. En appliquant la formule des traces de Grothendieck-Lefschetz, on obtient la formule

$$
\begin{aligned}
& \operatorname{Tr}\left(\sigma^{s} \circ\left(1 \otimes \cdots \otimes 1 \otimes \Phi_{\beta_{T^{\prime}}}\right), \operatorname{R\Gamma }\left(\left(\mathcal{S}_{\lambda}^{I}\right)^{r}(u), \mathcal{F}_{\lambda}^{\otimes r}\right)\right) \\
& \quad=\sum_{z \in \text { Fix }} \# \operatorname{Aut}(z)^{-1} \operatorname{Tr}\left(\sigma^{s} \circ\left(1 \otimes \cdots \otimes 1 \otimes \Phi_{\beta_{T^{\prime}}}\right),\left(\mathcal{F}_{\lambda}^{\bigotimes r}\right)_{z}\right)
\end{aligned}
$$

où Fix désigne l'ensemble des classes d'isomorphisme d'objets de la catégorie des points fixes $\operatorname{Fix}\left(\sigma^{s} \circ\left(1 \otimes \cdots \otimes 1 \otimes \Phi_{\beta_{T^{\prime}}}\right),\left(\mathcal{S}_{\lambda}^{I}\right)^{r}(u)\right)$.

Les objets de cette catégorie des points fixes sont constitués de

- $\widetilde{\mathcal{V}}_{i} \in \mathcal{D}$ - $\operatorname{Cht}_{\lambda}^{I}\left(\left(u_{i}\right)\right)$ pour tous $i=1, \ldots, r$,

- des isomorphismes $\widetilde{\mathcal{V}}_{2} \stackrel{\sim}{\longrightarrow} \sigma^{s}\left(\widetilde{\mathcal{V}}_{1}\right), \ldots, \widetilde{\mathcal{V}}_{r} \stackrel{\sim}{\longrightarrow} \sigma^{s}\left(\widetilde{\mathcal{V}}_{r-1}\right)$ et d'une $T^{\prime}$-modification $\sigma^{s}\left(\mathcal{V}^{r}\right)^{T^{\prime}} \stackrel{\sim}{\longrightarrow} \mathcal{V}_{1}^{T^{\prime}}$ qui en toute place $v \in\left|T^{\prime}\right|$ soit de type $\beta_{v}$.

Il revient au même de se donner un point $\widetilde{\mathcal{V}}=(\mathcal{V}, t, \iota) \in \mathcal{D}$ - $\operatorname{Cht}_{\lambda}^{I}\left(u_{1}\right)(k)$ et une $T^{\prime}$-modification $t^{\prime}:{ }^{\sigma^{r s}} \widetilde{\mathcal{V}}^{T^{\prime}} \rightarrow \widetilde{\mathcal{V}}^{T^{\prime}}$ qui ait le type $\beta_{v}$ en toute place $v \in\left|T^{\prime}\right|$.

Comme dans le paragraphe précédent, on a les assertions suivantes.

Proposition 1. - Soit $z$ un point fixe de $\sigma^{s} \circ\left(1 \otimes \cdots \otimes 1 \otimes \Phi_{\beta_{T^{\prime}}}\right)$ dans $\left(\mathcal{S}_{\lambda}^{I}\right)^{r}(u)$. ̀̀ $z$ est associé $(\mathcal{V}, t, \iota) \in \mathcal{D}$ - $\operatorname{Cht}_{\lambda}^{I}\left(u_{1}\right)(k)$ muni de $t^{\prime}:{ }^{\sigma^{r s}} \widetilde{\mathcal{V}}^{T^{\prime}} \rightarrow \widetilde{\mathcal{V}}^{T^{\prime}}$ comme précédemment. Alors on a la formule

$$
\operatorname{Tr}\left(\sigma^{s} \circ\left(1 \otimes \cdots \otimes 1 \otimes \Phi_{\beta_{T^{\prime}}}\right),\left(\mathcal{F}_{\underline{\lambda}}^{\bigotimes r}\right)_{z}\right)=P_{\lambda \alpha}\left(q^{r s}\right)
$$

où $\operatorname{inv}(t)=\alpha u_{1}$.

COROLlaire 2. - On a la formule

$$
\begin{aligned}
& \operatorname{Tr}\left(\sigma^{s} \circ\left(1 \times \cdots \times 1 \times \Phi_{\beta_{T^{\prime}}}\right), \operatorname{R\Gamma }\left(\left(\mathcal{S}_{\lambda}^{I}\right)^{r}(u),\left(\mathcal{F}_{\lambda}^{I}\right)^{\otimes r}\right)\right) \\
& \quad=\sum_{\alpha \leqslant \lambda} P_{\lambda \alpha}\left(q^{r s}\right) \# \mathfrak{C}_{\alpha_{u_{1}}, \beta_{T^{\prime}}}^{I}\left(T, T^{\prime} ; r s\right)
\end{aligned}
$$

où $\alpha_{u_{1}}$ est la fonction $\left|T \otimes_{\mathbb{F}_{q}} \mathbb{F}_{q^{r s}}\right| \rightarrow \mathbb{Z}_{+}^{d}$ qui prend la valeur $\alpha$ en $u_{1}$ et 0 ailleurs.

$4^{\text {e }}$ SÉRIE - TOME $39-2006-\mathrm{N}^{\circ} 2$ 
Les catégories

$$
\mathfrak{C}_{\alpha u_{1}, \beta_{T^{\prime}}}^{I}\left(T, T^{\prime} ; r s\right) \quad \text { et } \quad \mathfrak{C}_{\sum_{i=1}^{I}}^{I} \alpha u_{i}, \beta_{T^{\prime}}\left(T, T^{\prime} ; s\right)
$$

ne semblent pas équivalentes de façon naturelle. Néanmoins, la formule de comptage leur donne le même cardinal. En effet, en appliquant le théorème 1 de 4.7 , et en tenant compte du fait que l'extension $F \otimes_{\mathbb{F}_{q}} \mathbb{F}_{q^{r s}}$ de $F$ est totalement décomposée en $x$, on obtient la formule

$$
\# \mathfrak{C}_{\alpha_{u_{1}}, \beta_{T^{\prime}}}^{I}\left(T, T^{\prime} ; r s\right)=\sum_{\gamma_{0}} \operatorname{vol}\left(G_{\gamma_{0}}(F) a^{\mathbb{Z}} \backslash G_{\gamma_{0}}\left(\mathbb{A}_{F}\right)\right) \prod_{v \neq x} \mathbf{O}_{\gamma_{0}}\left(\phi_{\beta_{v}}\right) \mathbf{O}_{\gamma_{0}}\left(\phi_{\alpha_{x}}\right)
$$

où $\phi_{\alpha_{x}} \in \mathcal{H}\left(G\left(F_{x}\right)\right)$ est la fonction caractéristique de la $G\left(\mathcal{O}_{x}\right)$-double classe correspondant à $\alpha_{x}$.

On en déduit l'égalité

$$
\operatorname{Tr}\left(\operatorname{Frob}_{[u]} \times(1 \otimes \cdots \otimes 1 \otimes f) \times,\left[A_{[u]}\right]\right)=\operatorname{Tr}\left(\operatorname{Frob}_{[u]} \times f,\left[B_{[u]}\right]\right)
$$

pour tous les points cycliques $u=\left(u_{i}\right)$. Le théorème 1 de $3.3 \mathrm{~s}$ 'en déduit par le théorème de densité de Chebotarev comme il a été expliqué dans 5.1.

\subsection{Calcul sur la petite diagonale : situation $A$}

Soit $x$ un point fermé de degré 1 de $X^{\prime}-I, \bar{x}$ le point géométrique au-dessus de $x$ et notons

$$
r \cdot \bar{x}:=(\underbrace{\bar{x}, \ldots, \bar{x}}_{r}) \in\left(X^{\prime}-I\right)^{r}(k)
$$

le point sur la petite diagonale correspondant.

La fibre $\left(\mathcal{S}_{\lambda}^{I}\right)^{r}(r . \bar{x})$ est la catégorie des $\left(\widetilde{\mathcal{V}}_{i}\right)_{i=1}^{r}$ où $\widetilde{\mathcal{V}}_{i}=\left(\mathcal{V}_{i}, t_{i}, \iota_{i}\right) \in \mathcal{S}_{\lambda}^{I}(\bar{x})$. Puisque $\bar{x}$ est défini sur $\mathbb{F}_{q}$, cette fibre a une $\mathbb{F}_{q}$-structure évidente. Elle est de plus munie d'un automorphisme $\tau$ d'ordre $r$ qui permute les facteurs de manière cyclique.

Soient $T^{\prime}$ un fermé de $X^{\prime}$ évitant $x$, et $\beta_{T^{\prime}}$ une fonction qui associe à toute $v \in\left|T^{\prime}\right|$ une double classe $\beta_{v} \in K_{v}^{I} \backslash G\left(F_{v}\right) / K_{v}^{I}$. Les points fixes de la correspondance $\tau \circ \sigma \circ\left(1 \times \cdots \times 1 \times \Phi_{\beta_{T^{\prime}}}\right)$ dans la fibre de $\left(\mathcal{S}_{\lambda}^{I}\right)^{r}(r . \bar{x})$ sont les $\left(\widetilde{\mathcal{V}}_{i}\right)_{i=1}^{r}$ munis

- des isomorphismes ${ }^{\sigma} \mathcal{V}_{1} \stackrel{\sim}{\longrightarrow} \mathcal{V}_{2}, \ldots,{ }^{\sigma} \mathcal{V}_{r-1} \stackrel{\sim}{\longrightarrow} \mathcal{V}_{r}$,

- d'une $T^{\prime}$-modification $t^{\prime}:{ }^{\sigma} \mathcal{V}_{r}^{\bar{T}^{\prime}} \stackrel{\sim}{\longrightarrow} \mathcal{V}_{1}^{\bar{T}^{\prime}}$, commutant à $t$, qui en toute place $v \in T^{\prime}$ ait le type $\beta_{v}$.

Il revient donc au même de donner $\mathcal{V} \in \mathcal{S}_{\lambda}^{I}(\bar{x})$ muni d'une modification $t^{\prime}: \sigma^{r} \mathcal{V}^{\bar{T}^{\prime}} \stackrel{\sim}{\longrightarrow} \mathcal{V}^{\bar{T}^{\prime}}$ qui en toute place $v \in T^{\prime}$ ait le type $\beta_{v}$.

COROLlaire 1. - On a

$$
\begin{aligned}
& \operatorname{Tr}\left(\tau \circ \sigma \circ\left(1 \times \cdots \times 1 \times \Phi_{\beta_{T^{\prime}}}\right),\left[A_{r \cdot \bar{x}}\right]\right) \\
& \quad=\sum_{\left(\gamma_{0}, \delta_{x}\right)} \operatorname{vol}\left(J_{\gamma_{0}}(F) a^{\mathbb{Z}} \backslash J_{\gamma_{0}}\left(\mathbb{A}_{F}\right)\right) \prod_{v \neq x} \mathbf{O}_{\gamma_{0}}\left(\phi_{\beta_{v}}\right) \mathbf{T} \mathbf{O}_{\delta_{x}}\left(\psi_{\lambda_{x \otimes_{\mathbb{F}_{q}} q^{r}}}\right)
\end{aligned}
$$

où

- $\left(\gamma_{0}, \delta_{x}\right)$ parcourt l'ensemble des paires constituées d'une classe de conjugaison $\gamma_{0}$ de $G(F)$ et d'une classe de $\sigma$-conjugaison

$$
\delta_{x} \in G\left(\left(F \otimes_{\mathbb{F}_{q}} \mathbb{F}_{q^{r}}\right)_{x}\right)
$$


dont la norme est $\gamma_{0}$,

- la fonction $\psi_{\lambda_{x \otimes_{\mathbb{R}_{q}} q^{r}}}$ est la fonction sphérique correspondant à la double classe $\lambda$ dans l'algèbre de Hecke $G\left(\left(F \otimes_{\mathbb{F}_{q}} \mathbb{F}_{q^{r}}\right)_{x}\right)$.

\subsection{Calcul sur la petite diagonale : situation $B$}

Soient $x, \bar{x}$ et $r . \bar{x}$ comme dans 5.5. On va maintenant calculer la trace sur la fibre de $B$ audessus du point $r . \bar{x}=(\bar{x}, \ldots, \bar{x})$; ce calcul est un peu plus difficile que dans le cas $A$ car on doit utiliser la théorie des modèles locaux et l'interprétation géométrique de l'homomorphisme de changement de base, voir [20].

Rappelons que l'action de $\langle\tau\rangle$ sur la fibre $[B]_{r . \bar{x}}$ a été obtenue par prolongement d'une action définie géométriquement sur restriction de $[B]$ sur l'ouvert $U$. A priori, on ne connaît pas la trace du Frobenius tordu par $\tau$ en dehors de l'ouvert $U$. Les modèles locaux sont utiles dans ces situations de mauvaise réduction.

Soit $X^{\prime[r]}=X^{\prime r} / \mathfrak{S}_{r}$ la $r$-ième puissance symétrique de $X^{\prime}$. Un $k$-point $\bar{T}$ de $X^{\prime[r]}$ est un diviseur effectif de degré $r$ de $X^{\prime}$. Pour tout $\lambda \in \mathbb{Z}_{+}^{d}$, on pose $\lambda \bar{T}:=\sum\left(m_{i} \lambda\right) x_{i}$ où $\bar{T}=\sum m_{i} x_{i}$. On a un morphisme

$$
c_{[r \lambda]}^{I}: \mathcal{S}_{[r \lambda]}^{I} \rightarrow X^{\prime[r]}
$$

où la fibre de $c_{[r \lambda]}^{I}$ au-dessus d'un point $\bar{T} \in X^{\prime[r]}$ est la catégorie en groupoïdes des triplets $(\mathcal{V}, t, \iota)$ où $\mathcal{V} \in \mathcal{D}-\operatorname{Fib}(k)$, où $t:{ }^{\sigma} \mathcal{V}^{\bar{T}} \stackrel{\sim}{\longrightarrow} \mathcal{V}^{\bar{T}}$ est une $\bar{T}$-modification d'invariant $\operatorname{inv}(t) \leqslant \lambda \bar{T}$ et où $\iota$ est une $I$-structure de niveau.

On a un diagramme commutatif

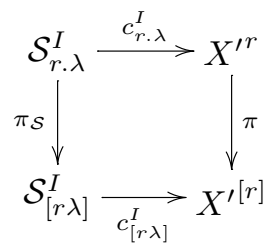

où $\pi_{\mathcal{S}}$ envoie un point

$$
\left(\left(x_{i}\right)_{i=1}^{r},\left(\mathcal{V}_{i}\right)_{i=0}^{r}, t_{i}: \mathcal{V}_{i}^{x_{i}} \stackrel{\sim}{\longrightarrow} \mathcal{V}_{i-1}^{x_{i}}, \varepsilon:{ }^{\sigma} \mathcal{V}_{0} \stackrel{\sim}{\longrightarrow} \mathcal{V}_{r}, \iota\right)
$$

de $\mathcal{S}_{r . \lambda}^{I}$ au-dessus de $\left(x_{1}, \ldots, x_{n}\right)$, sur le point $\left(t:{ }^{\sigma} \mathcal{V}_{0}^{\bar{T}} \rightarrow \mathcal{V}_{0}^{\bar{T}}, \iota\right)$ de $\mathcal{S}_{[r \lambda]}^{I}$ au-dessus de $\bar{T}=$ $x_{1}+\cdots+x_{r} \in X^{\prime(r)}$, où $t$ est la modification composée $t:=\varepsilon \circ t_{r} \circ \cdots \circ t_{1}$. D'après la propriété de factorisation du paragraphe 1.2, ce diagramme est cartésien au-dessus de l'ouvert $\pi(U)$ où $U$ est le complémentaire dans $X^{\prime r}$ de la réunion des diagonales.

On a de plus un diagramme cartésien

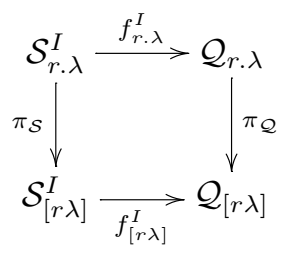

$4^{\text {e }}$ SÉRIE - TOME $39-2006-\mathrm{N}^{\circ} 2$ 
où $Q_{r . \lambda}$ est le champ des

$$
\left\{\left(x_{1}, \ldots, x_{r}\right) ;\left(0=Q_{0} \subset Q_{1} \subset Q_{2} \subset \cdots \subset Q_{r}\right)\right\}
$$

où $x_{1}, \ldots, x_{r} \in X^{\prime}$, où $Q_{i}$ sont des $\mathcal{D}$-modules de torsion tels que $Q_{i} / Q_{i-1}$ est supporté par $x_{i}$ et $\operatorname{inv}_{x_{i}}\left(Q_{i} / Q_{i-1}\right) \leqslant \lambda$. Le champ $\mathcal{Q}_{[r \lambda]}$ classifie les paires $(T, Q)$ où $T \in X^{(r)}$ et $Q$ est un $\mathcal{D}$-module de torsion tel que $\operatorname{inv}(Q) \leqslant \lambda T$. Le morphisme $\pi_{\mathcal{Q}}: \mathcal{Q}_{r . \lambda} \rightarrow \mathcal{Q}_{[r \lambda]}$ est défini par

$$
\left\{\left(x_{1}, \ldots, x_{r}\right) ;\left(0=Q_{0} \subset Q_{1} \subset Q_{2} \subset \cdots \subset Q_{r}\right)\right\} \mapsto(T, Q)
$$

$\operatorname{avec} T=x_{1}+\cdots+x_{r}$ et $Q=Q_{r}$.

PROPOSITION 1. - Le morphisme $\pi_{\mathcal{Q}}: \mathcal{Q}_{r . \lambda} \rightarrow \mathcal{Q}_{[r \lambda]}$ est un morphisme petit au sens stratifié. En particulier, l'image directe $\mathrm{R}\left(\pi_{\mathcal{Q}}\right)_{*} \mathcal{A}_{r . \lambda}$ est un faisceau pervers prolongement intermédiaire de sa restriction à l'ouvert où il est un système local.

Notons que la notion du morphisme petit au sens stratifié de Mirkovic et Vilonen, pour les schémas, voir [19], est conservé par un changement de base lisse. Il est donc bien défini pour les champs d'Artin. Il revient donc au même de démontrer la proposition après un changement de base lisse surjectif comme dans 1.1.5 pour se ramener aux grassmanniennes affines auquel cas elle est démontrée dans [19,21].

Au-dessus de l'ouvert $\pi(U)$, le diagramme

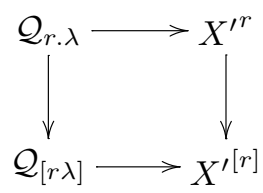

est cartésien de sorte qu'on a une action de $\mathfrak{S}_{r}$ sur la restriction de $\mathrm{R}\left(\pi_{\mathcal{Q}}\right)_{*} \mathcal{A}_{r . \lambda}$ à l'image inverse de $\pi(U)$ dans $\mathcal{Q}_{[r \lambda]}$. On en déduit une action de $\mathfrak{S}_{r}$ sur tout le faisceau pervers $\mathrm{R}\left(\pi_{\mathcal{Q}}\right)_{*} \mathcal{A}_{r . \lambda}$ par fonctorialité du prolongement intermédiaire.

D'après [20], l'action induite de la permutation cyclique $\tau$ sur ce faisceau pervers est reliée à l'homomorphisme de changement de base d'algèbres de Hecke. La fibre de $\mathcal{Q}_{[r \lambda]}$ au-dessus du point $T=r x$ a les points $Q_{\alpha(x)}$ paramétrés par $\alpha \leqslant r \lambda$, tous définis sur $\mathbb{F}_{q}$. On définit une fonction de l'algèbre de Hecke $\mathcal{H}_{x}$ en prenant la combinaison linéaire

$$
\psi_{[r \lambda](x)}=\sum_{\alpha \leqslant r \lambda} \operatorname{Tr}\left(\sigma \circ \tau,\left(\mathrm{R}\left(\pi_{\mathcal{Q}}\right)_{*} \mathcal{A}_{r . \lambda}\right)_{Q_{\alpha(x)}}\right) \phi_{\alpha_{x}}
$$

où $\phi_{\alpha_{x}} \in \mathcal{H}\left(G\left(F_{x}\right)\right)$ est la fonction caractéristique de la double classe correspondant à $\alpha$. On a l'énoncé suivant, voir [20, théorème 3].

PROPOSITION 2. - Soit y un point fermé de $X \otimes_{\mathbb{F}_{q}} \mathbb{F}_{q^{r}}$ au-dessus de $x$. Soient $\mathcal{H}_{y}$ l'algèbre de Hecke du groupe $G\left(\left(F \otimes_{\mathbb{F}_{q}} \mathbb{F}_{q^{r}}\right)_{y}\right)$ et $\mathbf{b}: \mathcal{H}_{y} \rightarrow \mathcal{H}_{x}$ l'homomorphisme de changement de base. Alors on a l'égalité

$$
\psi_{[r \lambda](x)}=\mathbf{b}\left(\psi_{\lambda(y)}\right)
$$


Avant de continuer notre discussion, rappelons qu'on a un diagramme :

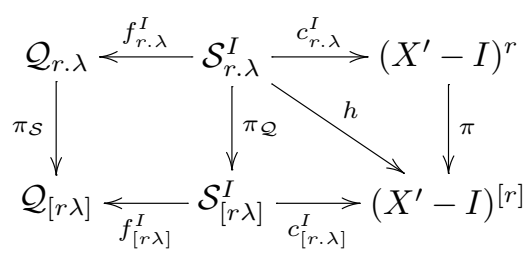

dont le carré de gauche est cartésien et dont le carré de droite est commutatif. Notons $h$ la flèche diagonale du carré de droite.

Le complexe d'intersection $\mathcal{F}_{r . \lambda}^{I}=\left(f_{r . \lambda}^{I}\right)^{*} \mathcal{A}_{r . \lambda}$ étant localement acyclique par rapport au morphisme $c_{r . \lambda}^{I}: \mathcal{S}_{r . \lambda}^{I} \rightarrow\left(X^{\prime}-I\right)^{r}$, les faisceaux de cohomologie

$$
B_{i}=\mathrm{R}^{i}\left(c_{r . \lambda}^{I}\right)_{*} \mathcal{F}_{r . \lambda}^{I}
$$

sont des systèmes locaux. Rappelons qu'on a une action de $\mathfrak{S}_{r}$ sur la restriction de $B_{i}$ à l'ouvert $U$ compatible à l'action de ce groupe sur $U$. Cette action s'étend à tout $B_{i}$ qui est un système local.

Puisque $\pi:\left(X^{\prime}-I\right)^{r} \rightarrow\left(X^{\prime}-I\right)^{[r]}$ est un morphisme fini, on a

$$
\mathrm{R}^{i} h_{*} \mathcal{F}_{r . \lambda}^{I}=\pi_{*} B_{i} .
$$

En particulier, $\mathrm{R}^{i} h_{*} \mathcal{F}_{r . \lambda}^{I}$ est muni d'une action de $\mathfrak{S}_{r}$ déduite de celle sur $B_{i}$.

Par ailleurs, $\mathrm{R} h_{*} \mathcal{F}_{r . \lambda}^{I}=\mathrm{R} c_{[r \lambda], *}^{I} \mathrm{R}\left(\pi_{\mathcal{Q}}\right)_{*} \mathcal{F}_{r . \lambda}^{I}$ où $\mathrm{R}\left(\pi_{\mathcal{Q}}\right)_{*} \mathcal{F}_{r . \lambda}^{I}=\left(f_{[r . \lambda]}^{I}\right)^{*} \mathcal{A}_{[r . \lambda]}$, de sorte que $\mathrm{R}\left(\pi_{\mathcal{Q}}\right)_{*} \mathcal{F}_{r . \lambda}^{I}$ hérite l'action canonique de $\mathfrak{S}_{r}$ sur le faisceau pervers $\mathcal{A}_{[r . \lambda]}$. On en déduit donc une action de $\mathfrak{S}_{r}$ sur tout le complexe $\mathrm{R} h_{*} \mathcal{F}_{r . \lambda}^{I}$, et en particulier sur ses faisceaux de cohomologie.

Proposition 3. - Les deux actions de $\mathfrak{S}_{r}$ sur $\mathrm{R}^{i} \chi_{*} \mathcal{F}_{r . \lambda}^{I}$ construites ci-dessus sont identiques.

Démonstration. - Soit $j$ l'immersion ouverte de l'image $\pi(U)$ dans $\left(X^{\prime}-I\right)^{[r]}$. Puisque $\pi$ est un morphisme fini, on a

$$
\pi_{*} B_{i}=j_{*} j^{*} \pi_{*} B_{i}
$$

où $j_{*}$ est le foncteur image directe sur les faisceaux ordinaires. Il suffit alors de vérifier que les deux actions de $\mathfrak{S}_{r}$ coïncident sur $\pi(U)$ ce qui est une tautologie.

Au-dessus du point $[r \bar{x}]$ image de $r \cdot \bar{x} \in X^{\prime^{r}}$ dans $X^{\prime^{[r]}}$ le morphisme fini $\pi: X^{\prime^{r}} \rightarrow X^{\prime^{[r]}}$ est complètement ramifié c'est-à-dire que l'image inverse de $[r \bar{x}]$ est un épaississement de $r . \bar{x}$. Par conséquent la fibre de $\pi_{*} B_{i}$ en $[r \bar{x}]$ est canoniquement isomorphe à la fibre de $B_{i}$ en $r \cdot \bar{x}$. La compatibilité entre les deux actions de $\langle\tau\rangle$ démontrée dans la proposition précédente, implique l'égalité

$$
\operatorname{Tr}\left(\tau \circ \sigma \circ \Phi_{\beta_{T^{\prime}}},\left[B_{r . \bar{x}}\right]\right)=\operatorname{Tr}\left(\tau \circ \sigma \circ \Phi_{\beta_{T^{\prime}}}, \mathrm{R} \Gamma\left(\mathcal{S}_{[r \lambda]}^{I}([r \bar{x}]), \mathrm{R}\left(\pi_{\mathcal{S}}\right)_{*} \mathcal{F}_{r . \lambda}^{I}\right)\right)
$$

où dans le membre droit l'action de $\tau$ provient de l'action triviale sur l'espace $\mathcal{S}_{[r \lambda]}^{I}([r \bar{x}])$ et de l'action non triviale de $\tau$ sur le faisceau pervers $\mathrm{R}\left(\pi_{\mathcal{S}}\right)_{*} \mathcal{F}_{r . \lambda}^{I}$ provenant de l'action de $\tau$ sur $\mathrm{R}\left(\pi_{\mathcal{Q}}\right)_{*} \mathcal{A}_{r . \lambda}$.

$4^{\text {e }}$ SÉRIE - TOME $39-2006-\mathrm{N}^{\circ} 2$ 
On est maintenant en position d'appliquer la formule des traces de Grothendieck-Lefschetz

$$
\begin{aligned}
& \operatorname{Tr}\left(\tau \circ \sigma \circ \Phi_{\beta_{T^{\prime}}}, \operatorname{R\Gamma }\left(\mathcal{S}_{[r \lambda]}^{I}([r \bar{x}]), \mathcal{F}_{[r \lambda]}^{I}\right)\right) \\
& \quad=\sum_{z \in \operatorname{Fix}\left(\tau \circ \sigma \circ \Phi_{\beta_{T^{\prime}}}\right)} \# \operatorname{Aut}(z) \operatorname{Tr}\left(\tau \circ \sigma \circ \Phi_{\beta_{T^{\prime}}},\left(\mathrm{R}\left(\pi_{\mathcal{S}}\right)_{*} \mathcal{F}_{r . \lambda}^{I}\right)_{z}\right) .
\end{aligned}
$$

Si $z=\left(\mathcal{V}, t, t^{\prime}, \iota\right)$, on sait que $\operatorname{Tr}\left(\tau \circ \sigma \circ \Phi_{\beta_{T^{\prime}}},\left(\mathrm{R}\left(\pi_{\mathcal{S}}\right)_{*} \mathcal{F}_{r . \lambda}^{I}\right)_{\left(\mathcal{V} ; t, t^{\prime}\right)}\right)$ ne dépend que de l'invariant $\alpha=\operatorname{inv}(t)$ et est égal au nombre

$$
\operatorname{Tr}\left(\tau \circ \sigma,\left(\mathrm{R}\left(\pi_{\mathcal{Q}}\right)_{*} \mathcal{A}_{r . \lambda}\right)_{\alpha(x)}\right)
$$

qui est un coefficient de $\mathbf{b}\left(\psi_{\lambda_{x}}\right)$ exprimé comme combinaison linéaire des $\phi_{\alpha(x)}$ d'après la proposition 2. On déduit la formule suivante du théorème 1 de 4.7 et de la proposition 2 de cette section.

Corollaire 4. - On a

$$
\begin{aligned}
& \operatorname{Tr}\left(\tau \circ \sigma \circ \Phi_{\beta_{T^{\prime}}},\left[B_{r . \bar{x}}\right]\right) \\
& =\sum_{\gamma_{0}} \operatorname{vol}\left(G_{\gamma_{0}}(F) a^{\mathbb{Z}} \backslash G_{\gamma_{0}}\left(\mathbb{A}_{F}\right)\right) \prod_{v \neq x} \mathbf{O}_{\gamma_{0}}\left(\phi_{\beta_{v}}\right) \mathbf{O}_{\gamma_{0}}\left(\mathbf{b}\left(\psi_{\lambda_{x}}\right)\right)
\end{aligned}
$$

où la fonction $\psi_{\lambda_{x}}$ est celle définie dans le corollaire 1 de 5.5 .

En appliquant le théorème 1 de 3.3, et en tenant compte du fait que les fonctions $\phi_{\beta_{v}}$ peuvent être prises arbitrairement, on obtient la formule.

Corollaire 5. - Soit $\bigotimes_{v \neq x} \phi_{v}$ une fonction telle que presque partout $\phi_{v}$ est l'unité de l'algèbre de Hecke; alors on a l'égalité

$$
\begin{aligned}
& \sum_{\left(\gamma_{0}, \delta_{x}\right)} \operatorname{vol}\left(J_{\gamma_{0}, \delta_{x}}(F) a^{\mathbb{Z} \backslash} \backslash J_{\gamma_{0}, \delta_{x}}\left(\mathbb{A}_{F}\right)\right) \prod_{v \neq x} \mathbf{O}_{\gamma_{0}}\left(\phi_{v}\right) \mathbf{T} \mathbf{O}_{\delta_{x}}\left(\psi_{\lambda_{x}}\right) \\
& =\sum_{\gamma_{0}} \operatorname{vol}\left(G_{\gamma_{0}}(F) a^{\mathbb{Z}} \backslash G_{\gamma_{0}}\left(\mathbb{A}_{F}\right)\right) \prod_{v \neq x} \mathbf{O}_{\gamma_{0}}\left(\phi_{v}\right) \mathbf{O}_{\gamma_{0}}\left(\mathbf{b}\left(\psi_{\lambda_{x}}\right)\right) .
\end{aligned}
$$

Notons qu'à la différence de la formule globale de Arthur et Clozel [1], en les places $v \neq x$, on n'a que des intégrales orbitales non tordues.

\subsection{Application au lemme fondamental}

Dans cette section, on va déduire du corollaire 5 de 5.6 le lemme fondamental pour le changement de base pour des fonctions de Hecke $\phi_{\lambda}$ avec $|\lambda|=0$ dans le cas de caractéristique positive.

THÉORÈmE 1.- Soient $F_{x}$ un corps local de caractéristique $p$ et $E_{x}$ son extension non ramifiée de degré $r$. Soient $\delta_{x}$ une classe de $\sigma$-conjugaison dans $\mathrm{GL}_{d}\left(E_{x}\right)$ et sa norme $\gamma_{x}$ une classe de conjugaison de $\mathrm{GL}_{d}\left(F_{x}\right)$. Supposons que $\gamma_{x}$ est une classe semi-simple régulière séparable. Alors pour toutes $\lambda \in \mathbb{Z}_{+}^{d}$ avec $|\lambda|=0$, on a

$$
\mathrm{TO}_{\delta_{x}}\left(\phi_{\lambda}\right)=\mathrm{O}_{\gamma_{x}}\left(\mathbf{b}\left(\phi_{\lambda}\right)\right)
$$

où $\phi_{\lambda}$ est la fonction caractéristique de la $\mathrm{GL}_{d}\left(\mathcal{O}_{E_{x}}\right)$-double classe correspondant à $\lambda$. 
Ce théorème a été démontré par Arthur-Clozel sans l'hypothèse $|\lambda|=0$ dans le cas $p$-adique à l'aide de la formule des traces. Il ne fait pas de doute que leur démonstration puisse être adaptée en caractéristique $p .^{2}$

Avec la méthode utilisée ici, on peut également se passer de l'hypothèse $|\lambda|=0$ au prix de quelques complications techniques. On a renoncé à cette généralisation dans la dernière version de ce texte.

Démonstration. - Soit $\mathbb{F}_{q}$ le corps résiduel de $F_{v}$. Soit $X$ une courbe projective lisse géométriquement connexe sur $\mathbb{F}_{q}$ avec un $\mathbb{F}_{q}$-point $x$ de sorte que le complété du corps des fonctions $F$ de $X$ en $v$ soit isomorphe au corps local $F_{v}$ de l'énoncé. L'extension $E_{x}$ est alors le complété en $x$ de $F \otimes_{\mathbb{F}_{q}} \mathbb{F}_{q^{r}}$. Notons $\bar{x}$ un point géométrique de $X$ au-dessus de $x$.

Soient $\gamma_{x}$ un élément semi-simple régulier séparable de $\operatorname{GL}\left(F_{v}\right)$ et $\delta_{x}$ un élément de $\operatorname{GL}\left(E_{v}\right)$ dont la norme est la classe de conjugaison de $\gamma_{x}$, comme dans l'énoncé.

On va choisir une algèbre à division $D$ de dimension $d^{2}$ sur son centre $F$ qui est non ramifié en $x$ mais qui a un nombre de places totalement ramifiées supérieur ou égal à $r d^{2}\|\lambda\|$. Sous cette hypothèse, on a des morphismes propres $c_{\lambda}^{I}: \mathcal{S}_{\lambda}^{I} \rightarrow\left(X^{\prime}-I\right)$ et $c_{r . \lambda}^{I}: \mathcal{S}_{r . \lambda}^{I} \rightarrow\left(X^{\prime}-I\right)^{r}$. On obtient alors l'égalité entre des sommes d'intégrales orbitales et intégrales orbitales tordues du corollaire 5 de 5.6.

Puisque les intégrales orbitales (resp. tordues) sont localement constantes aux voisinages des classes semi-simple régulières séparables, on peut trouver, par approximation faible, un élément $\gamma^{\prime} \in G(F)$ assez proche de $\gamma_{x}$ pour la topologie $x$-adique, qui est la norme d'un élément $\delta_{x}^{\prime} \in G\left(F_{x} \otimes_{\mathbb{F}_{q}} \mathbb{F}_{q^{r}}\right)$ et tel que $\mathbf{O}_{\gamma_{x}}\left(\mathbf{b}\left(\psi_{\lambda_{x}}\right)\right)=\mathbf{O}_{\gamma^{\prime}}\left(\mathbf{b}\left(\psi_{\lambda_{x}}\right)\right)$ et que $\mathbf{T} \mathbf{O}_{\delta_{x}}\left(\psi_{\lambda_{x}}\right)=\mathbf{T} \mathbf{O}_{\delta_{x}^{\prime}}\left(\psi_{\lambda_{x}}\right)$. Il suffit de démontrer que $\mathbf{O}_{\gamma^{\prime}}\left(\mathbf{b}\left(\psi_{\lambda_{x}}\right)\right)=\mathbf{T} \mathbf{O}_{\delta_{x}^{\prime}}\left(\psi_{\lambda_{x}}\right)$ car les fonctions $\psi_{\lambda_{x}}$ et les fonctions $\phi_{\lambda_{x}}$ engendrent le même espace vectoriel.

Dans le corollaire 5 de 5.6, on va choisir les fonctions $\phi_{v}$ en les autres places $v \neq x$ telles que

$$
\mathbf{O}_{\gamma^{\prime}}\left(\phi_{v}\right) \neq 0 \text {. }
$$

Pour ce choix, on a dans la somme le terme correspondant à $\gamma_{0}=\gamma^{\prime}$ et éventuellement un nombre fini d'autres classes de conjugaison $\gamma_{0}$. Quitte à rétrécir davantage le support de $\phi_{v}$, on peut se débarrasser de ces classes également tout en préservant la non-nullité $\mathbf{O}_{\gamma^{\prime}}\left(\phi_{v}\right) \neq 0$. Il ne reste alors plus qu'à diviser des deux côtés de l'égalité par des facteurs $\mathbf{O}_{\gamma^{\prime}}\left(\phi_{v}\right)$ pour $v \neq x$ et trouver

$$
\mathbf{O}_{\gamma^{\prime}}\left(\mathbf{b}\left(\psi_{\lambda_{x}}\right)\right)=\mathbf{T} \mathbf{O}_{\delta_{x}^{\prime}}\left(\psi_{\lambda_{x}}\right)
$$

\section{Remerciements}

Ce travail a été réalisé en grande partie durant une visite à l'IHÉS de 2001 à 2003. Je remercie A. Genestier, R. Kottwitz, L. Lafforgue, V. Lafforgue et G. Laumon pour leurs conseils et leurs encouragements, tout particulièrement $\mathrm{E}$. Lau pour sa vigilance. J'ai été très influencé par certaines idées de Rapoport sur les modèles locaux des variétés de Shimura. Je suis particulièrement reconnaissant au rapporteur de cet article pour sa lecture attentive et pour sa grande patience.

\section{RÉFÉRENCES}

[1] Arthur J., Clozel L., Simple Algebras, Base Change, and the Advaced Theory of the Trace Formula, Ann. of Math. Stud., vol. 120, Princeton University Press, Princeton, NJ, 1989.

\footnotetext{
${ }^{2}$ Cette démonstration est connue de Henniart qui ne l'a pas rédigée.

$4^{\mathrm{e}}$ SÉRIE - TOME $39-2006-\mathrm{N}^{\circ} 2$
} 
[2] Beauville A., Laszlo Y., Un lemme de descente, C. R. Acad. Sci. 320 (3) (1995) 335-340.

[3] Beilinson A., Drinfeld V., Quantization of Hitchin's Hamiltonian and Hecke's eigensheaves, Preprint.

[4] Clozel L., The fundamental lemma for stable base change, Duke Math. J. 61 (1990) 255-302.

[5] Drinfeld V., Moduli varieties of F-sheaves, Funct. Anal. Appl. 21 (1987) 23-41.

[6] GAitsGory D., Construction of central elements in the affine Hecke algebra via nearby cycles, Invent. Math. 144 (2001) 253-280.

[7] GinzBurg V., Perverse sheaves on Grassmannian and Langlands duality, Preprint arXiv.

[8] Grothendieck A., Dieudonné J., Éléments de géométrie algébrique I. Le langage des schémas, Publ. math. IHÉS, vol. 4, 1960.

[9] Grothendieck A., Revêtement étale et groupe fondamental, SGA 1, 1960-61, Documents mathématiques, Soc. Math. France, 2003.

[10] Kotтwitz R., Base change for unit elements of Hecke algebra, Compositio Math. 60 (1986) 237-250.

[11] Kotтwitz R., Points on some Shimura varieties over finite fields, J. Amer. Math. Soc. 5 (1992) 373444.

[12] Kottwitz R., Rogawski J., The distributions in the invariant trace formula are supported on characters, Canad. J. Math. 52 (2000) 804-814.

[13] Labesse J.-P., Fonctions élémentaires et lemme fondamental pour le changement de base, Duke Math. J. 61 (1990) 519-530.

[14] Lafforgue L., Chtoucas de Drinfeld et conjecture de Ramanujan-Peterson, Astérisque 243 (1997).

[15] Lafforgue L., Chtoucas de Drinfeld et correspondance de Langlands, Invent. Math. 147 (2002) $1-241$.

[16] LAUMON G., Cohomology of Drinfeld's modular varieties I, II, Cambridge University Press, Cambridge, 1996.

[17] Laumon G., Rapoport M., Stuhler U., D-elliptic sheaves and the Langlands correspondence, Invent. Math. 113 (1993) 217-338.

[18] Lusztig G., Singularities, character formulas and $q$-analog of weight multiplicities, Astérisque 101102 (1983) 208-229.

[19] Mirkovic I., Vilonen K., Perverse sheaves on affine Grassmannian and Langlands duality, Math. Res. Lett. 7 (2000) 13-24.

[20] NGÔ B.C., Faisceaux pervers, homomorphismes de changement de base et lemme fondamental de Jacquet et Ye, Ann. Sci. ÉNS 32 (1999) 619-679.

[21] NGô B.C., Polo P., Résolution de Demazure affine et formule de Casselman-Shalika géométrique, J. Alg. Geom. 10 (2001) 515-547.

[22] Pappas G., Rapoport M., Local models in the ramified case. I. The EL-case, J. Alg. Geom. 12 (2003) 107-145.

[23] RAPOPORT M., A guide to the reduction modulo $p$ of Shimura varieties, Preprint arXiv.

[24] SERrE J.-P., Prolongement de faisceaux analytiques cohérents, Ann. Inst. Fourier 16 (1966) 363-374.

[25] Shintani T., Two remarks on irreducible characters of finite general groups, J. Math. Soc. Japan 28 (1976) 396-414.

[26] VARShAVSKY Y., Moduli space of F-sheaves, Selecta Math. 10 (2004) 131-166.

(Manuscrit reçu le 15 décembre 2003; accepté, après révision, le 6 décembre 2005.)

\footnotetext{
Bao Châu NGÔ

Département de mathématiques,

Université Paris-Sud,

Bâtiment 425,

91405 Orsay, France

E-mail : bao-chau.ngo@math.u-psud.fr
} 